\title{
Risk aversion and equilibria, intertemporal transfers, strategic claims.
}

Citation for published version (APA):

Berden, C. (2008). Risk aversion and equilibria, intertemporal transfers, strategic claims. [Doctoral Thesis, Maastricht University]. Universiteit Maastricht. https://doi.org/10.26481/dis.20081209cb

Document status and date:

Published: 01/01/2008

DOI:

10.26481/dis.20081209cb

Document Version:

Publisher's PDF, also known as Version of record

\section{Please check the document version of this publication:}

- A submitted manuscript is the version of the article upon submission and before peer-review. There can be important differences between the submitted version and the official published version of record.

People interested in the research are advised to contact the author for the final version of the publication, or visit the DOI to the publisher's website.

- The final author version and the galley proof are versions of the publication after peer review.

- The final published version features the final layout of the paper including the volume, issue and page numbers.

Link to publication

\footnotetext{
General rights rights.

- You may freely distribute the URL identifying the publication in the public portal. please follow below link for the End User Agreement:

www.umlib.nl/taverne-license

Take down policy

If you believe that this document breaches copyright please contact us at:

repository@maastrichtuniversity.nl

providing details and we will investigate your claim.
}

Copyright and moral rights for the publications made accessible in the public portal are retained by the authors and/or other copyright owners and it is a condition of accessing publications that users recognise and abide by the legal requirements associated with these

- Users may download and print one copy of any publication from the public portal for the purpose of private study or research.

- You may not further distribute the material or use it for any profit-making activity or commercial gain

If the publication is distributed under the terms of Article $25 \mathrm{fa}$ of the Dutch Copyright Act, indicated by the "Taverne" license above, 


\section{Risk Aversion and Equilibria, Intertemporal Transfers, Strategic Claims}

Caroline Dorothea Anna-Maria Berden 
Magnolia soulangeana (watercolor) on cover by Caroline Berden

This book was typeset using LaTeX.

All rights reserved. No part of this publication may be reproduced, stored in a retrieval system, or transmitted, in any form, or by any means, electronic, mechanical, photocopying, recording or otherwise, without the prior permission in writing from the author.

Risk Aversion and Equilibria, Intertemporal Transfers, Strategic Claims

(c) Caroline Dorothea Anna-Maria Berden, 2008

ISBN 978-90-9023485-4

Printed by PrintPartners Ipskamp 


\title{
Risk Aversion and Equilibria, Intertemporal Transfers, Strategic Claims
}

\author{
Proefschrift \\ ter verkrijging van de graad van doctor \\ aan de Universiteit Maastricht, \\ op gezag van de Rector Magnificus, Prof. mr. G.P.M.F.Mols, \\ volgens het besluit van het College van Decanen, \\ in het openbaar te verdedigen \\ op dinsdag 9 december 2008 om 14:00 uur \\ door
}

Caroline Dorothea Anna-Maria Berden 


\section{Promotor:}

Prof. dr. H.J.M. Peters

\section{Copromotor:}

Dr. A.J. Vermeulen

\section{Beoordelingscommissie:}

Prof. dr. R.J. Müller (voorzitter)

Dr. B. Klaus

Prof. dr. H.W. Norde (Universiteit van Tilburg) 
To Stella, a best friend 



\section{Contents}

Acknowledgements $\quad$ v

1 Introduction 1

1.1 Overview of Chapter $2 \ldots \ldots \ldots 2$

1.2 Overview of Chapter $3 \ldots \ldots \ldots \ldots$

1.3 Overview of Chapter $4 \ldots \ldots \ldots \ldots$

1.4 Overview of Chapter $5 \ldots \ldots \ldots$

2 Risk Aversion in Bimatrix Games 11

2.1 Introduction .................... 11

2.2 Preliminaries ....................... 12

$2.32 \times 2$-Bimatrix Games . . . . . . . . . . . . . . . . . 14

2.4 Coordination Games . . . . . . . . . . . . . . 17

2.5 Concluding Remarks . . . . . . . . . . . . . . . 19

3 Risk Aversion in 2-Person, 2-State Finance Economies 21

3.1 Introduction . . . . . . . . . . . . . . 21

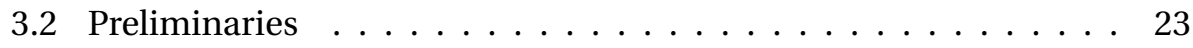

3.3 The Effect of Risk Aversion on Equilibrium . . . . . . . . . . 29

3.4 Concluding Remarks . . . . . . . . . . . . . . . 36

4 Intertemporal Transfers in Dynamic TU-Games 39

4.1 Introduction . . . . . . . . . . . . . . . 39

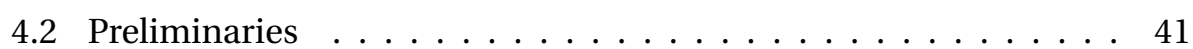

4.3 Individual Intertemporal Transfers . . . . . . . . . . . . . . . 44 
4.4 Pareto Optimality . . . . . . . . . . . . . . . . . . . 56

4.5 Concluding Remarks . . . . . . . . . . . . . . . . . . 57

5 Non-Cooperative Solutions for Claims Problems 59

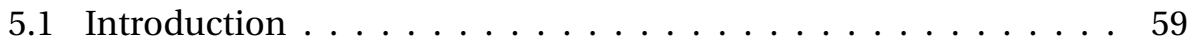

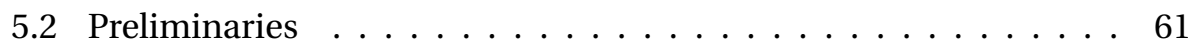

5.3 Some general results . . . . . . . . . . . . . . . . . . 64

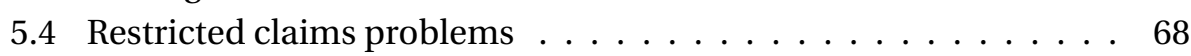

5.5 General claims problems $\ldots \ldots \ldots \ldots \ldots \ldots$

5.5 .1 Asymptotic result . . . . . . . . . . . . . . 75

5.6 Variations on the basic model . . . . . . . . . . . . . . 78

5.6 .1 Variation $1 \ldots \ldots \ldots \ldots \ldots$

5.6 .2 Variation $2 \ldots \ldots \ldots \ldots \ldots \ldots$

5.6 .3 Variation $3 \ldots \ldots \ldots \ldots \ldots$

5.6 .4 Variation $4 \ldots \ldots \ldots \ldots \ldots \ldots$

5.7 Conclusions and further research $\ldots \ldots \ldots \ldots$

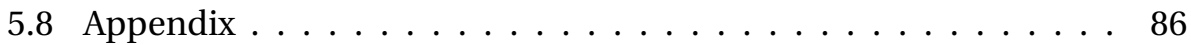

Nederlandse Samenvatting (Summary in Dutch) 91

$\begin{array}{ll}\text { Bibliography } & 95\end{array}$

$\begin{array}{ll}\text { Curriculum Vitae } & 99\end{array}$ 


\section{Tables}

1.1 Coalitional monetary gains $(0 \leq x \leq 100) \ldots \ldots 6$

1.2 Shapley values and Nucleoli $(x=0) \ldots \ldots$. . . . . . . 8

1.3 Shapley values and Nucleoli $(x=100) \ldots \ldots . \ldots$

4.1 Transfers with nucleolus values . . . . . . . . . . . . . . 52

4.2 Utility function player $1 \ldots \ldots \ldots 5$

4.3 Utility function player $2 \ldots \ldots \ldots 5$

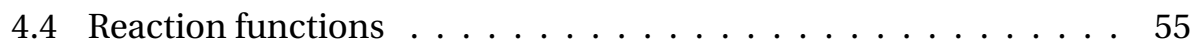

5.1 Marginal loss on all possible intervals (I) $\ldots \ldots$. . . . . . . 70

5.2 Marginal gain on all possible intervals (I) . . . . . . . . . . 71

5.3 Marginal loss on all possible intervals (II) . . . . . . . . . . . . . . . . . . 74

5.4 Marginal gain on all possible intervals (II) . . . . . . . . . . . . . . . . 74

5.5 Part of the marginal gain table . . . . . . . . . . . . . 90 



\section{Acknowledgements}

This dissertation presents the results of four and a half years of research in the field of game theory. Although pursuing a Ph.D. is often considered an isolated and individual activity, I can confirm the opposite. This project would not have been possible without the enormous help and support of a number of people whom I would like to acknowledge here.

Foremost, I owe special thanks to my promotor Hans Peters, whose direction and advice were indispensable to this work. The weekly meetings to discuss my findings were always very useful. With your astonishing knowledge in the field of game theory and your incredible patience, you gave me new insights which helped me to continue my research. Although a challenge, it certainly motivated me to come up with new results.

I am also greatly indebted to my co-promotor Dries Vermeulen. Your creativity in generating ideas and results amazes me. Moreover, I am thankful for your eagerness to help me at any point in time if and when I got stuck.

I thank the reading committee for their time and willingness to read the manuscript. Your remarks greatly helped in writing this final version.

I thank my colleagues for the relaxed atmosphere in which I was happy to work.

Thank you Mom and Dad. You were always there and supported me when I needed it.

And finally Brett, thank you for your patience, encouragement and support in all of my pursuits. 



\section{Introduction}

As indicated in its title, "Risk Aversion and Equilibria, Intertemporal Transfers, Strategic Claims", this dissertation deals with a number of topics rooted in game theory. This field of science mathematically studies the behavior of actors (players) in a situation in which a decision is to be taken. Our focus is on non-cooperative game theory. This means that each player has a strategy set and the overall solution depends on the chosen strategies of all players. Players can communicate, but they cannot make binding agreements. We assume that players are rational and therefore each player will choose a strategy which maximizes his expected utility given the other players' taken strategies.

The part "Risk Aversion and Equilibria" in the title refers to Chapters 2 and 3. Risk aversion is a concept related to the behavior of players in decision situations under uncertainty. A risk averse decision-maker will, when he has the choice between a risky and a risk free alternative with the same expected payoff, choose the risk free alternative. Ann is said to be more risk averse than Bill if and only if Bill prefers at least the same risky alternatives as Ann over all risk free alternatives. This is similar to the statement that Ann's utility function is a concave transformation of Bill's utility function. Or similarly, the Arrow-Pratt measure of risk aversion (Arrow [2]; Pratt [31]) is higher for Ann than for Bill for all risky alternatives.

The parts "Intertemporal Transfers" and "Strategic Claims" in the title relate to respectively Chapters 4 and 5 and refer to the way in which a strategic model is imposed on the cooperative game described in these chapters. In Chapter 4 we consider dynamic cooperative games with transferable utility. In a game with transferable utility (TU-game) it is implicitly assumed that the earnings to a coalition can be expressed by one number (a utility) and can be distributed (transferred) among the players in any possible way if the coalition is formed. A 
strategy represents a player's decision on how much value to transfer from one TU-game to other TU-games over time. In Chapter 5 we look at claims problems. In a claims problem every player has a claim on an estate, but the estate is not large enough to satisfy all claimants. A strategy represents a player's decision on how to place his claim on the estate. In both chapters we look, among others, under which conditions a Nash equilibrium exists in these superimposed models.

The best way to understand game theory is by the use of examples. Therefore, each chapter is shortly introduced, after which one of the results is illustrated by an economic example.

\subsection{Overview of Chapter 2}

In Chapter 2, of which the results are published in Berden and Peters [7], we compare Nash equilibria with identical supports for bimatrix games which are different with respect to the risk aversion of player 2 . We consider equilibria in $2 \times 2$-bimatrix games and equilibria with efficient supports in coordination games. For these equilibria we establish for which cases increased risk aversion of player 2 benefits or hurts player 2 .

Example 1. Congestion not only causes severe air pollution and noise that damages the happiness and health of individuals. The delays from congestion also cause economic costs to society. Think for example of the number of hours people spend in traffic not being productive. One way the government can deal with these external effects from congestion (and pollution) is by imposing an externality charge. This means that the individual who causes the traffic jam, that is the individual who goes to work by car, pays the cost he causes to society. Suppose in our example the government is deciding whether to impose a congestion tax. Moreover, we consider a particular individual from society, called Ann, who has to decide between going to work by car or by train. The following situations can occur:

(I) The government does not impose a tax and Ann drives to work by car.

(II) The government does not impose a tax and Ann takes the train to work.

(III) The government imposes a tax and Ann drives to work by car.

(IV) The government imposes a tax and Ann takes the train to work. 
The utility which the government enjoys if situation (I) occurs is equal to zero, Ann's utility in this situation equals 4 . Situation (II) gives the government a utility level of 5 and Ann a utility level of 1. Etc.

The above described problem can be modeled as follows:

$$
\begin{array}{cc}
\text { Car Train } \\
\text { Congestion Tax } \\
\left.\begin{array}{cc}
2,0 & 3,3 \\
0,4 & 5,1
\end{array}\right)
\end{array}
$$

This matrix represents a game with two players, the government and Ann. Each player has two possible choices. The government chooses a row and Ann a column. We assume that these choices are made simultaneously and independent of each other. The numbers represent the utility to the government and Ann. At each position the first number is the utility level for the government, the second number is the utility level for Ann. It is clear that no player has a dominant strategy, i.e. there is no choice which yields one player strictly highest utility levels independent of what the other player chooses. Furthermore, there is no Nash equilibrium in pure strategies. This simply means that there is no situation in which each player's choice is optimal given the choice of the other player. To see this, suppose Ann drives her car to work, then the government's best choice is to impose a tax, which leads to situation (III). However if the government imposes a tax, then Ann's best choice is to go to work by train, which is situation (IV). If Ann takes the train to work, then the government's best choice is to not impose a tax, which leads to situation (II). If the government does not impose a tax, then Ann's best choice is to go to work by car, which is situation (I).

Suppose players can randomize over their strategies, i.e they can use mixed strategies. Nash [28] proved that a Nash equilibrium in mixed strategies always exist in games where players have finitely many choices. It can be checked that if the government 'imposes a tax' and 'does not impose a tax' both with probability $\frac{1}{2}$, then for Ann it is optimal to randomize over her strategies 'car' and 'train'. Similarly, if Ann 'drives by car' and 'takes the train' both with probability $\frac{1}{2}$, then for the government it is optimal to randomize over her strategies 'congestion tax' and 'no congestion tax'. A different way of interpreting this Nash equilibrium in mixed strategies is to say that if Ann believes that the government 'imposes a tax' and 'does not impose a tax' both with probability $\frac{1}{2}$, then the government's optimal belief is that Ann randomizes over her strategies 'car' and 'train' each with probability $\frac{1}{2}$, and vice versa. In this case the expected payoff for Ann is equal to $\frac{1}{2} \cdot \frac{1}{2}(0+1+3+4)=2$. 
Assume Ann hates even more than before the uncertainty of not knowing whether the government is going to impose a congestion tax. Let's refer to this new Ann as 'Cautious' Ann. The Ann we originally considered will be referred to simply as Ann. In other words, 'Cautious' Ann is more risk averse than Ann. The higher level of risk aversion of 'Cautious' Ann can be represented by a concave transformation on Ann's utility function. It can be shown that if situations (I) and (III) yield 'Cautious' Ann the same utility as Ann, but situations (II) and (IV) yield her a utility of respectively $1 \frac{1}{2}$ and $3 \frac{1}{2}$, then 'Cautious' Ann is more risk averse than Ann. In order to see this, consider the risky alternative represented by the lottery where with probability $\frac{4}{5}$ situation (I) occurs and probability $\frac{1}{5}$ situation (III) occurs. Ann's expected utility of this lottery is equal to $\frac{4}{5} \cdot 4+\frac{1}{5}$. $0=3 \frac{1}{5}$. Ann prefers the described risky alternative over risk free alternative situation (IV) which gives her a utility of 3 . On the other hand, 'Cautious' Ann prefers risk free alternative situation (IV) which gives her a utility of $3 \frac{1}{2}$ over the described risky alternative. This reflects that 'Cautious' Ann is more risk averse than Ann. The problem can be summarized as follows:

$$
\begin{array}{cc}
\text { Car } & \text { Train } \\
\text { No Congestion Tax } & \left(\begin{array}{cc}
2,0 & 3,3 \frac{1}{2} \\
0,4 & 5,1 \frac{1}{2}
\end{array}\right)
\end{array}
$$

The matrix represents the decision situation between the government and 'Cautious' Ann. The government chooses a row and 'Cautious' Ann chooses a column. Again, it is easy to see that there is no pure Nash equilibrium. It can be checked that in the mixed Nash equilibrium the government believes that 'Cautious' Ann will 'drive her car' and will 'take the train' both with probability $\frac{1}{2}$, and 'Cautious' Ann believes that the government will 'impose a tax' with probability $\frac{5}{12}$ and will 'not impose a tax' with probability $\frac{7}{12}$.

This is very useful information to Ann. She can take advantage of the government by pretending to be 'Cautious' Ann. This means that Ann pretends to be more risk averse than she actually is. The government thinks she is facing 'Cautious' Ann and acts accordingly with the wrong beliefs (see the second matrix). As a result Ann's expected payoff from pretending to be 'Cautious' Ann is equal to $\frac{1}{2} \cdot \frac{7}{12} \cdot 4+\frac{1}{2} \cdot \frac{5}{12} \cdot 3+\frac{1}{2} \cdot \frac{7}{12} \cdot 1+\frac{1}{2} \cdot \frac{5}{12} \cdot 0=2 \frac{1}{12}$. Ann's expected payoff from pretending to exhibit more risk aversion equals $2 \frac{1}{12}$, whereas her expected payoff equals 2 in case she is honest about her level of risk aversion. In this example Ann benefits from pretending to be more risk averse. 


\subsection{Overview of Chapter 3}

In Chapter 3, of which the main results are published in Berden and Peters [8], we study the effect of replacing an agent in a two-person two-state finance economy by a more risk averse agent. We establish under which conditions the other agent benefits or loses in equilibrium from dealing with a more risk averse agent. If one agent becomes more risk averse, then the equilibrium allocation moves towards that agent's certainty line. Whether or not that is beneficial for the other agent, depends on the location of the endowment point.

Example 2. We apply our model to a situation with one health insurance company and one client. There are two possible states of the world which can occur. In one state the client gets ill, in the other state the client remains healthy. The state of the world is unknown to both the insurance company and the client at the point in time the client takes out a health insurance policy.

Ann wants to take out a health insurance policy, because she would like to insure herself against the financial cost involved with medical treatment in case she gets ill. Suppose that there is only one health insurance company in this society who sells one type of insurance. The insurance company is willing to insure Ann and they agree on a contract: Ann pays the insurance company 80 euros a month and in return the insurance company insures her. Consider the more risk averse 'Cautious' Ann, she hates the uncertainty of not knowing whether she is going to be ill or not even more than Ann. As a result she is willing to pay a higher risk premium for her insurance in order to not have to bear uncertainty. Clearly, this is beneficial to the insurance company, because she will receive more than 80 euros for insuring 'Cautious' Ann.

It seems trivial and intuitive that a health insurance company always benefits from increased risk aversion of her clients, by receiving a higher price for the insurance policy. However, if we assume that besides the client, also the health insurance company exhibits risk aversion, we should not take this conclusion so quickly. The assumption makes for another rather surprising result which is described in Chapter 3.

\subsection{Overview of Chapter 4}

A dynamic TU-game consists of a finite player set, a finite sequence of TUgames and a profile of intertemporal utility functions. A solution can be ap- 
plied to every TU-game, resulting in a stream of payoffs for every player. A player evaluates his stream of payoffs according to his intertemporal utility function. A limitation of this static framework is that it does not capture dynamic features. Therefore we introduce in Chapter 4 a model, briefly discussed in Kranich et al. [24], in which players can change the amount available over time by transferring payoffs across periods. Each player faces individual transfer decisions which affect the sequence of TU-games and as a result affect the stream of payoffs to every player. Hence, we associate with each dynamic TUgame and (restrictively) additive solution a noncooperative game which models the strategic possibilities from these individual transfers. Conditions are established under which a Nash equilibrium exists. We show when a Nash equilibrium in dominant strategies is Pareto optimal.

Example 3. Ann, Bill and Clark are consultants in a consulting agency. A firm has hired them to work on two projects, denoted project 1 and project 2 . The projects have to be finished in a year from now. Project 1 is worked on and advice is given before project 2 is started. The money which the firm pays for the advice on every project is paid out immediately upon completion of a project to the consultant(s) who worked on it. If Ann were to work on project 1 and project 2 by herself, she would generate advice which would be valued in total 100 euros by the firm. If Bill were to work by himself on project 1 , the advice would be valued 200 euros by the firm. He lacks the experience to work alone on project 2, however he can contribute to project 2 when working with another consultant. If Ann and Bill were to work together on project 1, they would be paid 400 euros for their advice. Etc.

The above described situation can be modeled by two TU-games shown in Table 1.1. Game 1 [Game 2] shows for every coalition of consultants the monetary gains from working on project 1 [project 2]. For simplicity we refer to Ann as $A$, Bill as $B$ and Clark as $C$. A coalition of Ann and Bill is then denoted by $A B$. Similarly, the coalition where all consultants work together is denoted by $A B C$.

Table 1.1: Coalitional monetary gains with $0 \leq x \leq 100$.

\begin{tabular}{l|rrrrrrr}
\hline Coalition & $A$ & $B$ & $C$ & $A B$ & $A C$ & $B C$ & $A B C$ \\
\hline Game 1: Gains in project 1 & $x$ & 200 & 300 & 400 & 500 & 500 & 700 \\
Game 2: Gains in project 2 & $100-x$ & 0 & 0 & 200 & 200 & 200 & 400 \\
\hline
\end{tabular}


The value 400 in column ' $A B$ ' and row 'Game 1' denotes the following: If Ann and Bill were to work together on project 1, then they would receive a payment of 400 euros from the firm for their advice. Consider the case where Ann were to work on project 1 and 2 all by herself, then she would receive 100 euros for her complete advice. There is no specification how much she receives for her advice on the individual projects. As a result Ann has some freedom on how much time to spend on the individual projects, if she were to work on them. It holds that the more time is spend on a project, the better the advice is. Of course there is a moment that advice cannot be improved upon anymore. A better advice generates higher gains. However, time is limited to one year. Ann could choose to allocate most of her time to work on project 1 , which means that $x=100$ or she could choose to allocate most of the time to work on project 2 , which means that $x=0$. Therefore, $0 \leq x \leq 100$.

As is usual in TU-games, we assume that the 'grand' coalition, in this case coalition $A B C$, is formed. This means that we assume that Ann, Bill and Clark will always work together to generate an advice on project 1 and project 2 . Clearly, as can be seen in Table 1.1, Ann, Bill and Clark receive in total 700 euros for their advice on project 1 and 400 euros for their advice on project 2. This leaves us with a distribution problem. Because how should these gains from project 1 and project 2 be distributed among the consultants? In other words, what is each consultant's bonus in the projects?

We use two different ways to distribute the gains to Ann, Bill and Clark. The Shapley value (Shapley [33]) looks at the bargaining power of each consultant. The resulting allocation gives to every consultant the average amount he contributes to the coalition $A B C$ if the consultants sequentially form this coalition in a random order. Another approach to determine each consultant's bonus is to use the nucleolus (Schmeidler [32]). This distribution of gains is such that the benefits of the least satisfied coalition are maximized.

Consider the situation where $x=0$. In this situation Ann would receive nothing for project 1 , if she were to work on it by herself. She would receive 100 euros for project 2 , if she were to work on it by herself. The Shapley values and Nucleoli if $x=0$ are given in Table 1.2. It can be seen from the table that Ann's bonus of working on project 1 equals $133 \frac{1}{3}$ euros and her bonus of working on project 2 equals $166 \frac{2}{3}$ euros according to the Shapley value. Using the nucleolus, Ann's bonuses for project 1 and 2 are equal to respectively 200 and 150 euros. 
Table 1.2: Shapley values and Nucleoli if $x=0$.

\begin{tabular}{ccccc}
\hline \multicolumn{2}{c}{ Shapley value } & & \multicolumn{2}{c}{ nucleolus } \\
\cline { 5 - 5 } Game 1 & Game 2 & & $\begin{array}{c}\text { Game } 1 \\
(700 \text { euros })\end{array}$ & $\begin{array}{c}\text { Game } 2 \\
(400 \text { euros })\end{array}$ \\
\hline (700 euros $)$ & $(400$ euros $)$ & & 200 & 150 \\
$133 \frac{1}{3}$ & $166 \frac{2}{3}$ & Ann's bonus & 200 & 125 \\
$233 \frac{1}{3}$ & $116 \frac{2}{3}$ & Bill's bonus & 300 & 125 \\
$333 \frac{1}{3}$ & $116 \frac{2}{3}$ & Clark's bonus & 300 \\
\hline
\end{tabular}

Next, consider the situation where $x=100$. Because of a different time allocation to the projects, Ann gains 100 euros, if she were to work on project 1 by herself. She does not receive any payment if she were to work on project 2 by herself. The Shapley values and Nucleoli if $x=100$ are given in Table 1.3.

Table 1.3: Shapley values and Nucleoli if $x=100$.

\begin{tabular}{ccccc}
\hline \multicolumn{2}{c}{ Shapley value } & & \multicolumn{2}{c}{ nucleolus } \\
\cline { 5 - 5 } Game 1 & Game 2 & & Game 1 & Game 2 \\
(700 euros) & $(400$ euros $)$ & & (700 euros) & $(400$ euros) \\
\hline $166 \frac{2}{3}$ & $133 \frac{1}{3}$ & Ann's bonus & 200 & $133 \frac{1}{3}$ \\
$216 \frac{2}{3}$ & $133 \frac{1}{3}$ & Bill's bonus & 200 & $133 \frac{1}{3}$ \\
$316 \frac{2}{3}$ & $133 \frac{1}{3}$ & Clark's bonus & 300 & $133 \frac{1}{3}$ \\
\hline
\end{tabular}

We assume that Ann prefers 1 euro now over 1 euro later. Note that the gains from project 1 (Game 1) are received before those of project 2 (Game 2). It follows that Ann prefers situation $x=100$ over $x=0$ in case the Shapley value is used to distribute the gains of the projects. Starting from the situation where $x=0$ and the Shapley value is used for distribution, Ann can gain by allocating her time differently to the projects. It is beneficial for her to transfer time and thus gains, from project 2 to project 1 . In case the nucleolus is used to compute the distribution, then clearly, she prefers situation $x=0$ over $x=100$. We conclude that in case the initial situation is $x=0$ and the nucleolus is used to compute the bonus allocation, Ann cannot gain by transferring time and thus 
gains from project 2 to project 1.

\subsection{Overview of Chapter 5}

In a claims problem an estate has to be divided among several claimants whose total claim exceeds the size of the estate. Chapter 5 extends the noncooperative approach, initiated by O'Neill [29], by allowing players to put multiple claims on the same part of the estate, and by considering the case where individual claims may exceed the estate. A full characterization of the set of Nash equilibria is obtained for restricted claims problems, where individual claims do not exceed the estate, and substantial results are obtained for the general case.

Example 4. Suppose that Ann, Bill and Clark have individually lent respectively 1000, 2000 and 5000 euros to a mutual friend. In other words, Ann, Bill and Clark are creditors. Tragically however their friend has passed away, leaving his debts and land of 60 square meters. The land is everywhere of the same quality. On the market 1 square meter of this type of land is sold for 100 euros. As a result Ann, Bill and Clark have claims over land of respectively 10, 20 and 50 square meters. Clearly, since there is only 60 square meters of land, there is not enough land to satisfy all creditors. Suppose that Ann, Bill and Clark can communicate, but cannot make binding agreements about the division of land. However, each of them knows that whatever action he or she takes, the overall solution depends on the actions taken by all of them. Assume that the land can be represented by the interval $[0,60]$. Each creditor can act by placing claims on pieces of the land in such a way that all these pieces together add up to at most the size of his original claim. This action is referred to as a strategy. If a piece of land is claimed by more creditors, then it is divided such that every creditor receives a part of the piece proportional to the number of claims he put on it.

In Chapter 5 we find necessary and sufficient conditions for a Nash equilibrium strategy profile. In our example these conditions are

(I) Creditors have in total placed 2 claims on 20 square meters.

(II) Creditors have in total placed 1 claim on 40 square meters.

(III) No creditor has more than one claim on a piece of land.

Consider the following strategy profile which satisfies (I), (II) and (III): 
- Ann claims interval $[0,10]$ once; $[10,60]$ is not claimed.

- Bill claims interval $[10,30]$ once; $[0,10]$ and $[30,60]$ are not claimed.

- Clark claims intervals $[0,20]$ and $[30,60]$ once; $[20,30]$ is not claimed.

Ann has placed her claim on a piece of land which is also claimed by Clark. She therefore receives $\frac{1}{2} \cdot 10=5$ square meters of land. Bill has placed part of his claim on a piece of land which is also claimed by Clark, the other part of his claim is placed on a piece of land only claimed by him. He receives $\frac{1}{2} \cdot 10+10=$ 15 square meters of land. Clark receives $\frac{1}{2} \cdot 20+30=40$ square meters of land.

It is easy to see that in any strategy profile which satisfies conditions (I), (II) and (III), Clark receives at least 40 square meters. But is it 'fair' that Clark receives in any case at least 40 square meters? In Chapter 5 we briefly get back to this question (see Example 14). We give a full characterization of Nash equilibria and the according payoff distributions in situations similar to this example. 
2

\section{On the Effect of Risk Aversion in Bimatrix Games}

\subsection{Introduction}

Uncertainty plays a central role in the theory of games, be it uncertainty about the parameters of the game-usually called incomplete information-or uncertainty about the actions of the opponent(s) — called strategic uncertainty by von Neumann and Morgenstern [36]. Following these authors, uncertainty is usually modelled by assuming the players to maximize expected utility. It is somewhat of a surprise that the effects of one of the most extensively studied characteristics of expected utility, namely the Arrow-Pratt measure of risk aversion (Arrow [2]; Pratt [31]), have received relatively little attention in game theory, with the exception of bargaining theory (starting with Kannai [21] and Kihlstrom et al. [22]).

In this chapter ${ }^{1}$ we study the effects of increased risk aversion in two-person noncooperative games with finite pure strategy sets, i.e., bimatrix games. Specifically, we consider the situation where player 2 is replaced by a more risk averse player and study the effect of this on Nash equilibrium. In doing so we assume that the supports of the Nash equilibria under comparison in the two situations-one with the less risk averse player 2 and the other one with the more risk averse player 2-do not change, in order to make a meaningful comparison. This implies that the equilibrium strategy of player 2 and therefore the expected payoff of player 1 does not change. This may appear peculiar but it is a direct consequence of the fact that in a mixed strategy Nash equilibrium a

\footnotetext{
${ }^{1}$ This chapter is based on Berden and Peters [7].
} 
player is indifferent between the pure strategies that are played with positive probability. Since it is meaningless to compare the expected payoffs of player 2 in the two situations, we compare the equilibria themselves. Specifically, we say that risk aversion benefits player 2 if the Nash equilibrium in the situation with the more risk averse player 2 would give the less risk averse player 2 a higher payoff. In the opposite case, we say that risk aversion hurts player 2 .

We give a complete analysis of the $2 \times 2$-case (Section 2.3), and of coordination games in which the players coordinate on Nash equilibria with efficient supports (Section 2.4). The analysis of the $2 \times 2$-case suggests that it is difficult to obtain results for the completely general case.

We identify cases where risk aversion benefits player 2-this happens in particular for Nash equilibria with efficient supports in coordination gamesand cases where risk aversion hurts player 2 . In the former case, as an alternative interpretation, it would be advantageous for player 2 if he could make player 1 believe that he is more risk averse than he actually is. In the latter case, in contrast, player 2 would want player 1 to believe that he is less risk averse than he is in reality. See also the discussion in Section 2.2.

After preliminaries in Section 2.2 we proceed with $2 \times 2$ games in Section 2.3 and coordination games in Section 2.4. Section 2.5 concludes.

\subsection{Preliminaries}

A bimatrix game is a pair $(A, B)$ of $m \times n$-matrices of real numbers. A (mixed) strategy for player 1 is an element $p \in \Delta^{m}:=\left\{x \in \mathbb{R}^{m} \mid x \geq 0, \sum_{i=1}^{m} x_{i}=1\right\}$. Similarly, a strategy for player 2 is an element $q \in \Delta^{n}$. The strategy profile $(p, q)$ results in the expected payoffs $p A q$ for player 1 and $p B q$ for player 2. A Nash equilibrium is a strategy profile $\left(p^{*}, q^{*}\right)$ such that $p^{*} A q^{*} \geq p A q^{*}$ for all $p \in \Delta^{m}$ and $p^{*} B q^{*} \geq p^{*} B q$ for all $q \in \Delta^{n}$. Let $a_{i j}\left(b_{i j}\right)$ denote the element in row $i$ and column $j$ of matrix $A(B)$. As is well known, $\left(p^{*}, q^{*}\right)$ is a Nash equilibrium if and only if

$$
\sum_{j=1}^{n} a_{i j} q_{j}^{*} \geq \sum_{j=1}^{n} a_{k j} q_{j}^{*} \text { for all } i \text { with } p_{i}^{*}>0 \text { and all } k=1, \ldots, m
$$

and

$$
\sum_{i=1}^{m} b_{i j} p_{i}^{*} \geq \sum_{i=1}^{m} b_{i k} p_{i}^{*} \text { for all } j \text { with } q_{j}^{*}>0 \text { and all } k=1, \ldots, n \text {. }
$$


Let $(A, B)$ be a bimatrix game and let $k$ be a concave and strictly increasing function defined on (at least) the interval $\left[\min \left\{b_{i j}\right\}, \max \left\{b_{i j}\right\}\right]$. Let $k(B)$ denote the matrix with element $k\left(b_{i j}\right)$ in row $i$ and column $j$. We say that player 2 in the game $(A, k(B))$ is more risk averse than player 2 in the game $(A, B)$ (cf. [31]).

Suppose, in this situation, that $\left(p^{*}, q^{*}\right)$ is a Nash equilibrium in $(A, B)$ and that $\left(\tilde{p}, q^{*}\right)$ is a Nash equilibrium in $(A, k(B))$ such that $p^{*}$ and $\tilde{p}$ have the same support, that is, $p_{i}^{*}>0 \Leftrightarrow \tilde{p}_{i}>0$ for all $i=1, \ldots, m$. We say that risk aversion benefits player 2 at $\left(p^{*}, q^{*}\right)$ and $\left(\tilde{p}, q^{*}\right)$ if

$$
\tilde{p} B q^{*} \geq p^{*} B q^{*}
$$

and that risk aversion hurts player 2 at $\left(p^{*}, q^{*}\right)$ and $\left(\tilde{p}, q^{*}\right)$ if

$$
\tilde{p} B q^{*} \leq p^{*} B q^{*} .
$$

In words, if risk aversion benefits [hurts] player 2, then the equilibrium in the game with the more risk averse player 2 is better [worse] for the less risk averse player 2 than the equilibrium in his own game.

Note that we assume that the two Nash equilibria under comparison are similar in the sense that their supports are the same. Hence, the players agree on the strategies that should be played with positive probability. This implies that player 2 should not (or, at least, must not) change his strategy ( $q^{*}$ in both equilibria), so that (2.1) is fulfilled in both $(A, B)$ and $(A, k(B))$. Player 1 , however, adapts his strategy from $p^{*}$ in $(A, B)$ to $\tilde{p}$ in $(A, k(B))$, in order for (2.2) to hold with $\tilde{p}$ and $k(B)$ instead of $p^{*}$ and $B$.

Also note that it does not make sense to compare expected payoffs $p^{*} B q^{*}$ and $\tilde{p} k(B) q^{*}$, since the numbers in $B$ and $k(B)$ represent the underlying preferences only up to a positive affine transformation.

An alternative justification for definitions (2.3) and (2.4) is that player 1 may have only limited information about the utility function of player 2 . Specifically, suppose that player 1 knows player 2's utility function up to an increasing concave transformation, i.e., up to player 2's risk attitude. To fix ideas, one might think of the matrices $B$ and $k(B)$ as representing the utilities of monetary outcomes. Player 1 knows these monetary outcomes but not the utilities attached to them by player 2. The inequalities in (2.3) and (2.4) reflect the possible consequences for player 2 in a Nash equilibrium if player 1 believes that player 2 is more risk averse than he actually is. For this interpretation we must implicitly assume that player 2 behaves myopically in the sense that he does 
not react optimally to the 'wrong' strategy of player 1 , that is, he does not play a strategy $\tilde{q}$ that maximizes his payoff $\tilde{p} B q$ over all $q \in \Delta^{n}$. Allowing for this possibility would only increase the effect in (2.3), but might undo the effect in (2.4). This assumption of myopic behavior makes sense if player 2 does not know that player 1 misjudges player 2's risk attitude. But even if player 2 tries to make player 1 believe that he (player 2) is more risk averse than he actually is-because risk aversion may benefit player 2-then he might still behave myopically in order not to reveal his true risk attitude, although making this argument precise would call for a dynamic model such as a repeated game.

The purpose of this chapter is to investigate in which cases risk aversion benefits or hurts player 2 . In the next section we analyze the $2 \times 2$-case, and in Section 2.4 we consider the special case of coordination games. As the $2 \times 2$ case will reveal, a complete analysis of the general case does not seem to be tractable.

\section{$2.32 \times 2$-Bimatrix Games}

We consider bimatrix games $(A, B)$ with $m=n=2$. Let $k$ be a strictly increasing concave transformation. For pure strategy Nash equilibria $\left(p^{*}, q^{*}\right)$ in $(A, B)$ we have, in the notation of the preceding section, $\tilde{p}=p^{*}$, so that (2.3) and (2.4) are fulfilled with equality. This case is not interesting and, in fact, we will assume, a forteriori, that player 2 has no (weakly) dominated strategy. For

$$
B=\left[\begin{array}{ll}
b_{11} & b_{12} \\
b_{21} & b_{22}
\end{array}\right]
$$

this implies that $b_{11} \neq b_{12}$. Without loss of generality we take $b_{11}>b_{12}$ and, thus, $b_{21}<b_{22}$. It is easy to verify that the following — not completely disjointcases are exhaustive:

(i) $b_{11}>b_{12} \geq b_{22}>b_{21}$;

(ii) $b_{11} \geq b_{22} \geq b_{12} \geq b_{21}$ and $b_{11}>b_{12}, b_{22}>b_{21}$;

(iii) $b_{11} \geq b_{22}>b_{21} \geq b_{12}$.

Let $\left(p^{*}, q^{*}\right)$ be a completely mixed Nash equilibrium in $(A, B)$, i.e. $0<p_{1}^{*}, q_{1}^{*}<$ 1. (Of course, the existence of such an equilibrium depends on $A$ as well, but 
will just be assumed here.) Then

$$
p_{1}^{*}=\frac{b_{22}-b_{21}}{b_{22}-b_{21}+b_{11}-b_{12}},
$$

as can easily be verified.

We first consider case (i), and normalize the function $k$ such that $k\left(b_{21}\right)=$ $b_{21}$ and $k\left(b_{11}\right)=b_{11}$. Hence, $k\left(b_{22}\right) \geq b_{22}$ and $k\left(b_{12}\right) \geq b_{12}$ by concavity of $k$. For the equilibrium $\left(\tilde{p}, q^{*}\right)$ in $(A, k(B))$ we now derive

$$
\tilde{p}_{1}=\frac{k\left(b_{22}\right)-b_{21}}{k\left(b_{22}\right)-b_{21}+b_{11}-k\left(b_{12}\right)} \geq p_{1}^{*} .
$$

In order to show that equation (2.6) is indeed true, assume to the contrary that $\tilde{p}_{1}<p_{1}^{*}$. It then follows that

$$
\frac{1}{\tilde{p}_{1}}>\frac{1}{p_{1}^{*}} \Leftrightarrow \frac{k\left(b_{22}\right)-b_{21}+b_{11}-k\left(b_{12}\right)}{k\left(b_{22}\right)-b_{21}}>\frac{b_{22}-b_{21}+b_{11}-b_{12}}{b_{22}-b_{21}},
$$

which is equivalent to

$$
\frac{b_{11}-k\left(b_{12}\right)}{k\left(b_{22}\right)-b_{21}}>\frac{b_{11}-b_{12}}{b_{22}-b_{21}} .
$$

However, this inequality causes a contradiction since $k\left(b_{12}\right) \geq b_{12}$ and $k\left(b_{22}\right) \geq$ $b_{22}$. As a result equation (2.6) is true.

Hence

$$
\begin{aligned}
\tilde{p} B q^{*} & =\tilde{p}_{1}\left[q_{1}^{*} b_{11}+q_{2}^{*} b_{12}\right]+\tilde{p}_{2}\left[q_{1}^{*} b_{21}+q_{2}^{*} b_{22}\right] \\
& \geq p_{1}^{*}\left[q_{1}^{*} b_{11}+q_{2}^{*} b_{12}\right]+p_{2}^{*}\left[q_{1}^{*} b_{21}+q_{2}^{*} b_{22}\right] \\
& =p^{*} B q^{*},
\end{aligned}
$$

where the inequality follows from (2.6) and the fact that $q_{1}^{*} b_{11}+q_{2}^{*} b_{12} \geq q_{1}^{*} b_{21}+$ $q_{2}^{*} b_{22}$. We conclude that, in case (i), risk aversion benefits player 2 at $\left(p^{*}, q^{*}\right)$ and $\left(\tilde{p}, q^{*}\right)$.

Consider next case (ii). The analysis of case (i) goes through including (2.6), but now there are two cases:

(a) $q_{1}^{*} b_{11}+q_{2}^{*} b_{12} \geq q_{1}^{*} b_{21}+q_{2}^{*} b_{22}$. In this case, risk aversion again benefits player 2 , just like in case (i), see (2.7).

(b) $q_{1}^{*} b_{11}+q_{2}^{*} b_{12} \leq q_{1}^{*} b_{21}+q_{2}^{*} b_{22}$. In this case we have the reverse inequality in (2.7), so that risk aversion hurts player 2 . 
So in case (ii), whether risk aversion benefits or hurts player 2 depends on $q^{*}$, hence on the matrix $A$. Since

$$
q_{1}^{*}=\frac{a_{22}-a_{12}}{a_{22}-a_{12}+a_{11}-a_{21}}
$$

risk aversion benefits player 2 (case (a)) if

$$
\frac{a_{22}-a_{12}}{a_{22}-a_{12}+a_{11}-a_{21}} \geq \frac{b_{22}-b_{12}}{b_{22}-b_{12}+b_{11}-b_{21}}
$$

and risk aversion hurts player 2 (case (b)) if

$$
\frac{a_{22}-a_{12}}{a_{22}-a_{12}+a_{11}-a_{21}} \leq \frac{b_{22}-b_{12}}{b_{22}-b_{12}+b_{11}-b_{21}} .
$$

Consider, finally, case (iii). For this case we show by means of an example that the effect of risk aversion is ambiguous and depends not only on the matrices $A$ and $B$ but also on the function $k$. Consider the bimatrix games

$$
(A, B)=\left[\begin{array}{ll}
0,7 & 1,0 \\
1,2 & 0,4
\end{array}\right], \quad\left(A, B^{\prime}\right)=\left[\begin{array}{ll}
0,5 & 1,0 \\
1,2 & 0,4
\end{array}\right], \quad\left(A, B^{\prime \prime}\right)=\left[\begin{array}{cc}
0,7 & 1,-1 \\
1,2 & 0,4
\end{array}\right] .
$$

Clearly, both $B^{\prime}$ and $B^{\prime \prime}$ can be obtained by applying increasing concave transformations to $B$. The (unique) Nash equilibrium in $(A, B)$ is $\left(p^{*}, q^{*}\right)=\left(\left(\frac{2}{9}, \frac{7}{9}\right)\right.$, $\left.\left(\frac{1}{2}, \frac{1}{2}\right)\right)$, in $\left(A, B^{\prime}\right)$ it is $\left(p^{\prime}, q^{*}\right)=\left(\left(\frac{2}{7}, \frac{5}{7}\right),\left(\frac{1}{2}, \frac{1}{2}\right)\right)$, and in $\left(A, B^{\prime \prime}\right)$ it is $\left(p^{\prime \prime}, q^{*}\right)=\left(\left(\frac{1}{5}, \frac{4}{5}\right)\right.$, $\left.\left(\frac{1}{2}, \frac{1}{2}\right)\right)$. Then $p^{*} B q^{*}=3 \frac{1}{9}, p^{\prime} B q^{*}=3 \frac{1}{7}$, and $p^{\prime \prime} B q^{*}=3 \frac{1}{10}$. Hence, risk aversion benefits player 2 at $\left(p^{*}, q^{*}\right)$ and $\left(p^{\prime}, q^{*}\right)$ but hurts player 2 at $\left(p^{*}, q^{*}\right)$ and $\left(p^{\prime \prime}, q^{*}\right)$.

The intuition for the different results in these three cases is as follows. In case (i), player 1 has to put more weight on the upper row in order to keep the more risk averse player 2 indifferent, but this row is unambiguously the best row from the point of view of player 2 , so risk aversion benefits player 2. In case (ii) player 1 again has to put more weight on the first row, but now it depends on $q^{*}$, and thus on the matrix $A$, whether the first or second row is better from the point of view of player 2 . This results in the two cases (a) and (b). In case (iii) it is not only ambiguous which one of the two rows is better from the point of view of player 2, but also the change in weight put by player 1 on the first row is ambiguous, as is clear from the example.

We summarize and slightly extend these results in the following two propositions. The first proposition summarizes cases (i) and (ii). 
Proposition 1. Let $m=n=2$, let $(A, B)$ and $(A, k(B))$ be bimatrix games with $k$ an increasing and concave function, and let $\left(p^{*}, q^{*}\right)$ and $\left(\tilde{p}, q^{*}\right)$ be completely mixed Nash equilibria in $(A, B)$ and $(A, k(B))$, respectively.

(i) If $b_{11}>b_{12} \geq b_{22}>b_{21}$, then risk aversion benefits player 2 at $\left(p^{*}, q^{*}\right)$ and $\left(\tilde{p}, q^{*}\right)$.

(ii) If $b_{11} \geq b_{22} \geq b_{12} \geq b_{21}$ and $b_{11}>b_{12}, b_{22}>b_{21}$, then risk aversion benefits player 2 at $\left(p^{*}, q^{*}\right)$ and $\left(\tilde{p}, q^{*}\right)$ if (2.8) holds and risk aversion hurts player 2 at $\left(p^{*}, q^{*}\right)$ and $\left(\tilde{p}, q^{*}\right)$ if (2.9) holds.

The second proposition considers case (iii).

Proposition 2. Let $m=n=2$, let $(A, B)$ be a bimatrix game, and let $\left(p^{*}, q^{*}\right)$ be a completely mixed Nash equilibrium. Let $b_{11} \geq b_{22}>b_{21} \geq b_{12}$. Then there are increasing concave transformations $k$ and $k^{\prime}$ and Nash equilibria $\left(\tilde{p}, q^{*}\right)$ in $(A, k(B))$ and $\left(\tilde{p}^{\prime}, q^{*}\right)$ in $\left(A, k^{\prime}(B)\right)$ such that risk aversion benefits player 2 at $\left(p^{*}, q^{*}\right)$ and $\left(\tilde{p}, q^{*}\right)$ but hurts player 2 at $\left(p^{*}, q^{*}\right)$ and $\left(\tilde{p}^{\prime}, q^{*}\right)$.

Proof. Let $k$ satisfy $k(x)=x$ for all $x \leq b_{22}$ and $k\left(b_{11}\right)=b_{22}+\frac{1}{2}\left(b_{11}-b_{22}\right)$. Then it is straightforward to verify (using (2.5)) that $\tilde{p}_{1} \geq p_{1}^{*}$. Let $k^{\prime}$ satisfy $k^{\prime}(x)=x$ for all $x \geq b_{21}$ and $k^{\prime}\left(b_{12}\right)=b_{12}-\frac{1}{2}\left(b_{21}-b_{12}\right)$. Then it is easy to verify (again using (2.5)) that $\tilde{p}_{1}^{\prime} \leq p_{1}^{*}$. If (2.8) holds, then $k$ and $k^{\prime}$ are as desired. If (2.9) holds, then the proof is complete by switching the roles of $k$ and $k^{\prime}$.

Proposition 2 in particular makes it clear that there is not much hope to obtain general results for the $m \times n$-case. In the next section we consider the special but interesting case of coordination games.

\subsection{Coordination Games}

A coordination game is an $m \times m$-bimatrix game $(A, B)$ such that $a_{i j}=b_{i j}=0$ whenever $i \neq j$, and $a_{i i}, b_{i i}>0$ for all $i=1, \ldots, m$. Clearly, the players would want to coordinate on one or more diagonal elements. In such a game, for each non-empty subset $I \subseteq\{1, \ldots, m\}$ there is a unique Nash equilibrium with support $I$ for each player, namely the strategy profile $\left(p^{I}, q^{I}\right)$ satisfying

$$
p_{i}^{I} b_{i i}=p_{j}^{I} b_{j j} \text { and } q_{i}^{I} a_{i i}=q_{j}^{I} a_{j j} \text { for all } i, j \in I, p_{k}=q_{k}=0 \text { for all } k \notin I .
$$


Observe that, for $m=2$, such a coordination game falls under case (ii) and under case (iii) in Section 2.3. In fact, it is a boundary case between these two: Proposition 2 applies, but the function $k^{\prime}$ defined in its proof is constant, so that it only depends on $q^{*}$ (i.e., on $A$ ) whether risk aversion benefits or hurts player 2 .

In a coordination game equilibria of interest are those where inefficient diagonal outcomes are played with zero probability. More generally, we say that a Nash equilibrium $\left(p^{I}, q^{I}\right)$ has efficient support if for all $i, j \in I, a_{i i}>a_{j j}$ implies $b_{i i}<b_{j j}{ }^{2}$ Clearly, if $\left(p^{I}, q^{I}\right)$ has efficient support and $k$ is an increasing concave function with $k(0)=0$, then the equilibrium $\left(\tilde{p}^{I}, q^{I}\right)$ in $(A, k(B))$ also has efficient support. ${ }^{3}$ Moreover, we have the following result.

Proposition 3. Let $(A, B)$ be an $m \times m$-coordination game and let, for some $I \subseteq\{1, \ldots, m\},\left(p^{I}, q^{I}\right)$ be a Nash equilibrium with efficient support in $(A, B)$. Let $k$ be an increasing concave function with $k(0)=0$ and let $\left(\tilde{p}^{I}, q^{I}\right)$ be a Nash equilibrium in $(A, k(B))$. Then risk aversion benefits player 2 at $\left(p^{I}, q^{I}\right)$ and $\left(\tilde{p}^{I}, q^{I}\right)$.

Proof. Without loss of generality suppose $I=\{1, \ldots, m\}$ and $0<b_{11} \leq \ldots \leq b_{m m}$. We write $p$ instead of $p^{I}, \tilde{p}$ instead of $\tilde{p}^{I}$, and $q$ instead of $q^{I}$.

Since $(p, q)$ has efficient support and by $(2.10)$, we have $0<q_{1} \leq \ldots \leq q_{m}$, so that

$$
0<q_{1} b_{11} \leq \ldots \leq q_{m} b_{m m} .
$$

By concavity of $k, k\left(b_{i i}\right) / b_{i i} \geq k\left(b_{j j}\right) / b_{j j}$ whenever $i \leq j$. By (2.10) this implies

$$
\frac{\tilde{p}_{i}}{\tilde{p}_{j}}=\frac{k\left(b_{j j}\right)}{k\left(b_{i i}\right)} \leq \frac{b_{j j}}{b_{i i}}=\frac{p_{i}}{p_{j}} \text { for all } i, j \in I \text { with } i \leq j .
$$

For all $\ell, j \in I$ with $\ell>j$, (2.12) implies

$$
\frac{p_{1}}{p_{\ell}}+\cdots+\frac{p_{j}}{p_{\ell}} \geq \frac{\tilde{p}_{1}}{\tilde{p}_{\ell}}+\cdots+\frac{\tilde{p}_{j}}{\tilde{p}_{\ell}}
$$

hence

$$
\frac{p_{\ell}}{p_{1}+\cdots+p_{j}} \leq \frac{\tilde{p}_{\ell}}{\tilde{p}_{1}+\cdots+\tilde{p}_{j}}
$$

\footnotetext{
${ }^{2}$ Clearly, unless $I$ is a singleton, such an equilibrium is neither ex ante nor ex post efficient. So the efficient support requirement is a very weak efficiency requirement.

${ }^{3}$ The condition $k(0)=0$ is imposed in order that $(A, k(B))$ is formally a coordination game. No generality is lost.
} 
hence

$$
\frac{p_{j+1}+\cdots+p_{m}}{p_{1}+\cdots+p_{j}} \leq \frac{\tilde{p}_{j+1}+\cdots+\tilde{p}_{m}}{\tilde{p}_{1}+\cdots+\tilde{p}_{j}}
$$

hence

$$
\frac{1-\left(p_{1}+\cdots+p_{j}\right)}{p_{1}+\cdots+p_{j}} \leq \frac{1-\left(\tilde{p}_{1}+\cdots+\tilde{p}_{j}\right)}{\tilde{p}_{1}+\cdots+\tilde{p}_{j}}
$$

which implies

$$
p_{1}+\cdots+p_{j} \geq \tilde{p}_{1}+\cdots+\tilde{p}_{j} \text { for all } j=1, \ldots, m .
$$

Define $b_{00}:=0$ and $q_{0}:=0$, then

$$
\begin{aligned}
\sum_{j=1}^{m} p_{j} q_{j} b_{j j} & =\sum_{j=1}^{m}\left(\sum_{i=j}^{m} p_{i}\right)\left(q_{j} b_{j j}-q_{j-1} b_{j-1, j-1}\right) \\
& \leq \sum_{j=1}^{m}\left(\sum_{i=j}^{m} \tilde{p}_{i}\right)\left(q_{j} b_{j j}-q_{j-1} b_{j-1, j-1}\right) \\
& =\sum_{j=1}^{m} \tilde{p}_{j} q_{j} b_{j j},
\end{aligned}
$$

where the inequality follows from (2.11) and (2.13). Now (2.14) says that risk aversion benefits player 2, as was to be proved.

As a note to this proof, observe that (2.13) states that $\tilde{p}$ first-degree stochastically dominates $p$, and (2.14) is the familiar consequence that the expected payoff under $\tilde{p}$ is higher than under $p$.

The intuition for Proposition 3 is that increased risk aversion of player 2 forces player 1 to put more weight on the outcomes that are better for player 2 . From the point of view of the original-less risk averse-player 2 the resulting distribution is preferable. This intuition (and result) is similar to the result established for a bargaining context in Köbberling and Peters [23], although that paper assumes rank-dependent utility instead of expected utility.

\subsection{Concluding Remarks}

This chapter has shown that at least in some interesting cases it is possible to say something about the effect of the risk attitude of a player in a noncooperative game. From a more general point of view the chapter can also be interpreted as studying the effect of one player having limited information about the 
utility function of another player. We focus, however, on risk attitude, which is one of the most important characteristics of an expected utility function. Further research may concentrate on specific economic games, such as risky investments in portfolios. 


\section{On the Effect of Risk Aversion in Two-Person, Two-State Finance Economies}

\subsection{Introduction}

The concept of risk plays a central role in financial markets, both in theory and in practice. The celebrated capital asset pricing model, for instance, gives a relation between equilibrium asset prices when - among other conditions agents' choices are based on a tradeoff between means and variances of assets. An aspect, however, which to the best of our knowledge has received little attention is the effect an agent's risk attitude has on the utility of the other agents in equilibrium. ${ }^{1}$ The purpose of this chapter ${ }^{2}$ is to study this effect in about the simplest possible model, namely a two-person two-state finance economy (Arrow-Debreu economy).

In such an economy the two agents are equipped with endowments of a single good (e.g., money) in two possible states of the world. These states of the world occur with commonly known probabilities. The agents trade in the two associated Arrow securities and evaluate allocations according to their von Neumann-Morgenstern utility functions. At the equilibrium prices, markets clear - the total allocation per state is equal to the total endowment per state while agents maximize their expected utility. This is the simplest version of a financial market (see, for instance, LeRoy and Werner [27]). It is equivalent to

\footnotetext{
${ }^{1}$ There is a literature on risk aversion of the representative consumer, see Section 3.4 for a brief discussion.

${ }^{2}$ This chapter is based on Berden and Peters [8].
} 
a two-person two-good exchange economy with, of course, quite special utility functions.

To facilitate the exposition we assume that the economy has a unique interior equilibrium and then we ask the following question. Suppose an agent is replaced by a more risk averse agent in the sense of the Arrow-Pratt measure of risk aversion (Arrow [2]; Pratt [31]): is the new equilibrium allocation better or worse for the other agent? In slightly different wording, does increased risk aversion of one agent benefit or hurt the other agent?

In answering this question, we find that the position of the endowment point is an important factor. If agent $A$ is replaced by a more risk averse agent, then the equilibrium allocation moves towards $A$ 's certainty line, i.e., the line of risk free allocations. This movement takes place along agent $B$ 's offer (demand) curve. If the new equilibrium allocation is farther away from the endowment point than the original one, then $B$ 's utility increases, otherwise it decreases. Only cases where the equilibrium allocation passes the endowment point are ambiguous in terms of the change of B's utility.

As a typical example, suppose the total endowment in state 1 is higher than in state 2, agent $A$ has a higher endowment in state 1 than in state 2 , and in equilibrium agent $A$ 's allocation in state 1 is smaller than his endowment in state 1. I.e., he has traded part of his state 1 endowment for state 2 securities. In that case, if agent $A$ is replaced by a more risk averse agent, that agent will demand even more state 2 securities in order to move closer to a risk free allocation. Hence, the equilibrium allocation moves away from the endowment point along $B$ 's offer curve. Such a change is always to the benefit of agent $B-$ intuitively since the endowment point is available whatever the prices are, so moving away from it reveals an increase in utility. This movement is accompanied by a price decrease of state 1 securities. The intuition that agent $B$ benefits from this is tempting, but it should be taken with some care since price changes also have income effects. Nevertheless, the situation can be summarized by saying that agent $B$ benefits from a more risk averse agent $A$ demanding a less risky portfolio, resulting in a price decrease of an asset demanded by $B$ and having a positive net effect on $B$ 's utility.

Other cases, collected in the main result Theorem 1 of this chapter, can be given similar interpretations. It may not come as a big surprise that it depends on the relative locations of the endowment and the equilibrium whether increased risk aversion of one agent benefits or hurts the other agent. On the 
other hand, for any possible configuration we can either say what is the case, or spell out why it is ambiguous. Our results are only for two agents and two states, but these can be interpreted as aggregates for two different groups of agents and two different classes of states. See also the concluding section of this chapter.

The chapter is organized as follows. Section 3.2 describes the model and preliminary observations, Section 3.3 presents the main result, Section 3.4 concludes.

\subsection{Preliminaries}

We consider economies with two agents $A$ and $B$, a single good, and two states of the world. Since the state of the world unfolds over time, we define two time periods; $t=0$ and $t=1$. At $t=0$ there is no information about the true state of the world, at $t=1$ uncertainty is resolved, i.e. the true state of the world is revealed (either state 1 or state 2 ). For simplicity we assume that consumption only takes place at $t=1$, there is no endowment at $t=0$. The endowments of the agents are denoted by $e^{A}=\left(e_{1}^{A}, e_{2}^{A}\right)$ and $e^{B}=\left(e_{1}^{B}, e_{2}^{B}\right)$, where the subscripts refer to the states of the world. Throughout, it is assumed that $e^{A}>0$ and $e^{B}>0$, i.e., each agent is endowed with a positive amount of the good in each state. ${ }^{3}$ State of the world 1 occurs with probability $\pi$ and state of the world 2 with probability $1-\pi$, where $0<\pi<1$. The total endowment in state 1 is denoted by $w_{1}$ and the total endowment in state 2 by $w_{2}$, so $w_{1}=e_{1}^{A}+e_{1}^{B}$ and $w_{2}=e_{2}^{A}+e_{2}^{B}$. The preferences of the agents are expressed by von Neumann-Morgenstern utility functions $u^{A}, u^{B}:[0, m] \rightarrow \mathbb{R}$, where $m \in \mathbb{R}$ with $m \geq \max \left\{w_{1}, w_{2}\right\}$. We assume that these functions are strictly concave, strictly increasing and twice differentiable on the interior of their domain, so $\left(u^{A}\right)^{\prime},\left(u^{B}\right)^{\prime}>0$ and $\left(u^{A}\right)^{\prime \prime},\left(u^{B}\right)^{\prime \prime}<0$. Such an economy is denoted by $\mathscr{E}=\left(u^{A}, u^{B}, e^{A}, e^{B}, \pi\right)$.

In this economy all trading takes place at $t=0$. Agents trade in Arrow securities, which transfer wealth across the states of the world revealed at $t=1$. An Arrow security promises to deliver 1 unit of the single good in a particular state at $t=1$ and zero in every other possible state. By $p_{s}$ we denote the price of the Arrow security for state $s \in\{1,2\}$. Price $p_{s}$ is also called a 'state' price; paying 1 unit of the single good in state $s$ and nothing in all other possible states. At $t=0$

\footnotetext{
${ }^{3}$ The vector inequality $x>y$ means that every coordinate of $x$ is strictly larger than the corresponding coordinate of $y$.
} 
each agent sells his endowment against state-prices, yielding an income with which he buys a new portfolio of Arrow securities. He evaluates a portfolio by first assigning to every state its probability. Then, the good at state $s$ is evaluated according to the agent's preferences at $t=0$. Finally, the agent computes the expected utility.

More formally, the trade decision process for every agent described above can be written down as follows. Let $p_{1}$ and $p_{2}$ be positive state prices. Given these prices, an agent in the economy with utility function $u$ and endowment $e=\left(e_{1}, e_{2}\right)$ faces the familiar consumer problem

$$
\begin{array}{r}
\max _{x_{1}, x_{2}} \pi u\left(x_{1}\right)+(1-\pi) u\left(x_{2}\right) \\
\text { subject to } p_{1} x_{1}+p_{2} x_{2}=p_{1} e_{1}+p_{2} e_{2} \\
x_{1}, x_{2} \geq 0 .
\end{array}
$$

From our assumptions it follows that this problem has a unique solution, the demand vector $x\left(p_{1}, p_{2}\right)$. An interior solution to (3.1) satisfies

$$
\frac{p_{1}}{p_{2}}=\frac{\pi u^{\prime}\left(x_{1}\right)}{(1-\pi) u^{\prime}\left(x_{2}\right)},
$$

with $x_{1}$ and $x_{2}$ satisfying the budget constraint. As is clear from the budget constraint and also from (3.2), prices are relative. It will often be convenient to fix the price of state 2 at $p_{2}=1$ and consider changes in $p_{1}$, or conversely.

A pair $x^{A}, x^{B} \in \mathbb{R}^{2}$ of nonnegative vectors is called an allocation. If, moreover, $x_{1}^{A}+x_{1}^{B}=w_{1}$ and $x_{2}^{A}+x_{2}^{B}=w_{2}$, then the allocation is called feasible. As usual, a (Walrasian) equilibrium in this economy is a vector of positive prices $p=\left(p_{1}, p_{2}\right)$ and a feasible allocation $x^{A}=\left(x_{1}^{A}, x_{2}^{A}\right)$ and $x^{B}=\left(x_{1}^{B}, x_{2}^{B}\right)$ such that $x^{A}$ solves (3.1) for $A$ and $x^{B}$ solves (3.1) for $B$. Feasibility implies that markets clear.

A useful instrument to study equilibria is the offer curve. For an agent with utility function $u$ and endowment $e$ in the economy $\mathscr{E}$, the offer curve associates with each price vector $p=\left(p_{1}, p_{2}\right)$ the demand vector $x(p) \in \mathbb{R}^{2}$ which solves problem (3.1). Clearly and well-known, by (3.2), the offer curve of an agent passes through the endowment, and in the economy $\mathscr{E}$, a feasible allocation $\left(x^{A}, x^{B}\right) \neq\left(e^{A}, e^{B}\right)$ is part of an equilibrium if and only if $x^{A}$ is on $A$ 's offer curve and $x^{B}$ is on $B$ 's offer curve. When drawn as curves in an Edgeworth box, both the offer curve of $A$ and of $B$ passes through the endowment point $e=\left(e^{1}, e^{2}\right)$ and through each equilibrium allocation $\left(x^{A}, x^{B}\right)$. We will use the expression 'offer curve' also for the corresponding curve in the Edgeworth box. 


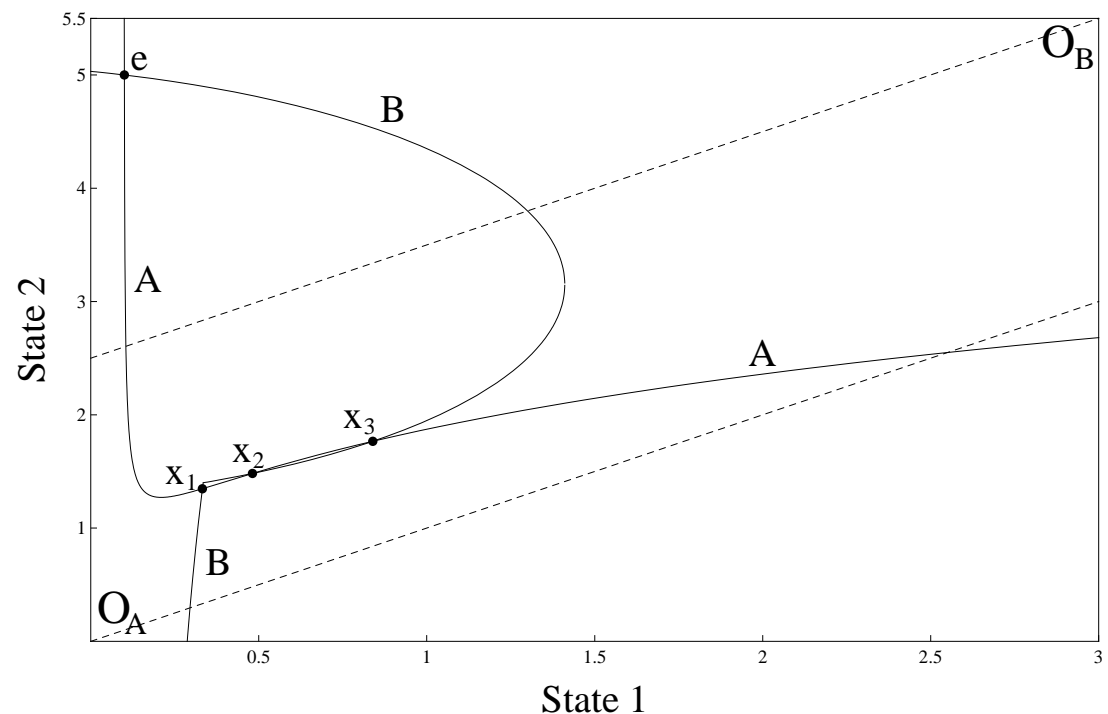

Figure 3.1: Three equilibria: $x_{1}=(0.33,1.35), x_{2}=(0.48,1.48)$ and $x_{3}=(0.84,1.77)$. 
To simplify matters we assume that the economy has a unique interior Walrasian equilibrium. Otherwise a unique solution is not guaranteed, as example 5 shows.

Example 5. Consider a two-agent, two-state economy in which both states are equally likely. Agent $A$ has utility function $u^{A}(t)=\alpha-\beta\left(t+\frac{1}{100}\right)^{-1}$ and endowment $e^{A}=\left(\frac{1}{10}, 5\right)$. Clearly, $\left(u^{A}\right)^{\prime}>0$ and $\left(u^{A}\right)^{\prime \prime}<0$. Agent $B$ has utility function

$$
u^{B}(t)= \begin{cases}\left(\frac{42}{10}\right)^{2}-\left(\frac{42}{10}-t\right)^{2} & \text { if } 0 \leq t \leq \frac{41}{10}, \\ \frac{4613}{250}-\frac{168921}{50000}\left(t+\frac{1}{100}\right)^{-1} & \text { if } t \geq \frac{41}{10} .\end{cases}
$$

His endowment is equal to $e^{B}=\left(\frac{29}{10}, \frac{1}{2}\right)$. It is clear that $u^{B}$ is continuous.

We show that $u^{B}$ is strictly increasing and strictly concave ${ }^{4}$. Consider functions $f_{1}, f_{2}:[0, \infty) \rightarrow \mathbb{R}$ of which $u^{B}$ is created, $f_{1}(t)=\left(\frac{42}{10}\right)^{2}-\left(\frac{42}{10}-t\right)^{2}$ is strictly increasing and strictly concave for $t \in\left[0, \frac{42}{10}\right)$ and $f_{2}(t)=\frac{4613}{250}-\frac{168921}{50000}\left(t+\frac{1}{100}\right)^{-1}$ is strictly increasing and strictly concave for $t \in[0, \infty)$. As a result $u^{B}$ is strictly increasing. In order to show that $u^{B}$ is strictly concave we define function $f:[0, \infty) \rightarrow \mathbb{R}$ with $f(t)=\min \left\{f_{1}(t), f_{2}(t)\right\}$ for all $t \in[0, \infty)$. It can be shown that $f$ is strictly concave. This means that between points $t^{\prime}$ and $t^{\prime \prime}$ with $t^{\prime}<\frac{41}{10}$ and $t^{\prime \prime}>\frac{41}{10}$, function $f$ is strictly concave. As a result $u^{B}$ is strictly concave.

Figure 3.1 is a graphical representation of the problem generated by computer program Mathematica. Besides the intersection in the endowment point, the offercurves of both players intersect in three other places denoted $x_{1}, x_{2}$ and $x_{3}$. This means that there are three equilibria.

For later reference we collect some useful observations about offer curves and equilibria. For completeness' sake, all proofs are provided.

The first lemma describes how demand and, thus, offer curves depend on prices.

Lemma 1. Consider an agent in $\mathscr{E}$ with utility function $u$ and endowment $e$. Let $x_{1}\left(p_{1}, p_{2}\right)$ and $x_{2}\left(p_{1}, p_{2}\right)$ be the demand functions in state 1 and state 2 , respectively. Assume interior demand, i.e., $x_{1}\left(p_{1}, p_{2}\right)>0$ and $x_{2}\left(p_{1}, p_{2}\right)>0$ for all $p_{1}, p_{2}>0$.

\footnotetext{
${ }^{4}$ Formally $u^{B}$ does not fit the assumptions of the model; it is not twice differentiable. However, the results are valid in a more general context which assumes only strict concavity and continuity of utility functions.
} 
(i) If $e_{1}-x_{1}\left(p_{1}, p_{2}\right) \leq 0$, then $\frac{d x_{1}}{d p_{1}}<0 .{ }^{5}$

(ii) If $e_{1}-x_{1}\left(p_{1}, p_{2}\right) \geq 0$, then $\frac{d x_{2}}{d p_{1}}>0$.

Proof. Since prices are relative, it is without loss of generality to set $p_{2}=1$. Then, by (3.2), for the demand $x(p)$ we have $p_{1}=\frac{\pi u^{\prime}\left(x_{1}\right)}{(1-\pi) u^{\prime}\left(x_{2}\right)}$, with $x_{2}=p_{1}\left(e_{1}-\right.$ $\left.x_{1}\right)+e_{2}$. By straightforward computation,

$$
\frac{d x_{1}}{d p_{1}}=\frac{(1-\pi)\left(u^{\prime}\left(x_{2}\right)\right)^{2}+\pi u^{\prime}\left(x_{1}\right) u^{\prime \prime}\left(x_{2}\right)\left(e_{1}-x_{1}\right)}{\pi\left(u^{\prime}\left(x_{2}\right) u^{\prime \prime}\left(x_{1}\right)+p_{1} u^{\prime}\left(x_{1}\right) u^{\prime \prime}\left(x_{2}\right)\right)} .
$$

This implies (i). For (ii), again by straightforward computation,

$$
\frac{d x_{2}}{d p_{1}}=\frac{\pi u^{\prime \prime}\left(x_{1}\right)\left(x_{2}-e_{2}\right)-p_{1}^{2}(1-\pi) u^{\prime}\left(x_{2}\right)}{p_{1}^{3}(1-\pi) u^{\prime \prime}\left(x_{2}\right)+p_{1} \pi u^{\prime \prime}\left(x_{1}\right)} .
$$

If $e_{1}-x_{1}\left(p_{1}, p_{2}\right) \geq 0$, then by the budget constraint $e_{2}-x_{2}\left(p_{1}, p_{2}\right)=e_{2}-x_{2} \leq 0$, so (ii) follows from (3.4).

An implication of Lemma 1 for the offer curve is given in the following lemma. The lemma says that an agent's offer curve cuts each budget line in two points, namely the endowment point and the demand point: for in-between prices the offer curve is below, and for other prices above the budget line.

Lemma 2. Consider an agent with utility function $u$ and endowment $e$. Fix the price of state 2 at $1, p_{2}=1$, and denote $x(p)$ by $x\left(p_{1}\right)$. Lete $=x\left(p_{1}^{e}\right)$ and let $q_{1} \neq p_{1}^{e}$ with $x\left(q_{1}\right)>0$. Then:

(i) if $p_{1}$ is weakly between $p_{1}^{e}$ and $q_{1}$, then $x\left(p_{1}\right)$ is affordable at price $q_{1}$, i.e.,

$$
q_{1} x_{1}\left(p_{1}\right)+x_{2}\left(p_{1}\right) \leq q_{1} e_{1}+e_{2} .
$$

(ii) if $p_{1}$ is not between $p_{1}^{e}$ and $q_{1}$, then $x\left(p_{1}\right)$ is not affordable at price $q_{1}$, i.e.,

$$
q_{1} x_{1}\left(p_{1}\right)+x_{2}\left(p_{1}\right)>q_{1} e_{1}+e_{2} .
$$

\footnotetext{
${ }^{5}$ Strictly speaking, these are partial derivatives. Since prices are relative, so that we can always fix the price of one of the goods (states), we use the ordinary derivative notation here and in the sequel.
} 
Proof. Assume without loss of generality that $x_{1}\left(q_{1}\right)<e_{1}$ and (thus) $x_{2}\left(q_{1}\right)>e_{2}$. Lemma 1 implies that $p_{1}^{e}<q_{1}$. To prove (i), let $p_{1}^{e} \leq p_{1} \leq q_{1}$. Then, since $e$ solves (3.1) at price $p_{1}^{e}$, we must have

$$
p_{1}^{e} x_{1}\left(p_{1}\right)+x_{2}\left(p_{1}\right) \geq p_{1}^{e} e_{1}+e_{2} .
$$

By subtracting this inequality from the equation

$$
p_{1} x_{1}\left(p_{1}\right)+x_{2}\left(p_{1}\right)=p_{1} e_{1}+e_{2}
$$

we obtain the inequality $\left(p_{1}-p_{1}^{e}\right) x_{1}\left(p_{1}\right) \leq\left(p_{1}-p_{1}^{e}\right) e_{1}$, which by $p_{1} \geq p_{1}^{e}$ implies $x_{1}\left(p_{1}\right) \leq e_{1} .{ }^{6}$ Suppose the statement in (i) were not true, i.e.,

$$
q_{1} x_{1}\left(p_{1}\right)+x_{2}\left(p_{1}\right)>q_{1} e_{1}+e_{2} .
$$

Then subtracting from this the equation $p_{1} x_{1}\left(p_{1}\right)+x_{2}\left(p_{1}\right)=p_{1} e_{1}+e_{2}$ yields $\left(q_{1}-p_{1}\right) x_{1}\left(p_{1}\right)>\left(q_{1}-p_{1}\right) e_{1}$, a contradiction since $p_{1} \leq q_{1}$ and $x_{1}\left(p_{1}\right) \leq e_{1}$.

The proof of part (ii) is similar and therefore omitted.

The final preliminary lemma establishes that an equilibrium allocation in our economy is always located between the agents' 'certainty lines.'?

Lemma 3. Let the interior demand vectors $x^{A}$ and $x^{B}$ with prices $\left(p_{1}, p_{2}\right)$ be an equilibrium in $\mathscr{E}$. Then,

(i) if $x_{1}^{A}=x_{2}^{A}$, then $x_{1}^{B}=x_{2}^{B}$ and hence $w_{1}=w_{2}$.

(ii) if $x_{1}^{A}>x_{2}^{A}$, then $x_{1}^{B}>x_{2}^{B}$ and hence $w_{1}>w_{2}$.

(iii) if $x_{1}^{A}<x_{2}^{A}$, then $x_{1}^{B}<x_{2}^{B}$ and hence $w_{1}<w_{2}$.

Proof. For case (i), suppose that $x_{1}^{A}=x_{2}^{A}$. Then by (3.2), $\frac{p_{1}}{p_{2}}=\frac{\pi}{(1-\pi)}$. Again by (3.2), this implies $x_{1}^{B}=x_{2}^{B}$. For case (ii), suppose that $x_{1}^{A}>x_{2}^{A}$. Then $\left(u^{A}\right)^{\prime}\left(x_{1}^{A}\right)<$ $\left(u^{A}\right)^{\prime}\left(x_{2}^{A}\right)$ and by (3.2), $\frac{p_{1}}{p_{2}}<\frac{\pi}{(1-\pi)}$. By (3.2) again, it follows that $\left(u^{B}\right)^{\prime}\left(x_{1}^{B}\right)<$ $\left(u^{B}\right)^{\prime}\left(x_{2}^{B}\right)$. Therefore, $x_{1}^{B}>x_{2}^{B}$. Case (iii) is analogous.

\footnotetext{
${ }^{6}$ If $p_{1}=p_{1}^{e}$ then $x_{1}\left(p_{1}\right)=e_{1}$ and so $x_{1}\left(p_{1}\right) \leq e_{1}$ as well.

${ }^{7}$ This is a special case of the well-known comonotonicity property of Pareto optimal allocations.
} 


\subsection{The Effect of Risk Aversion on Equilibrium}

Consider two economies $\mathscr{E}=\left(u^{A}, u^{B}, e^{A}, e^{B}, \pi\right)$ and $\widetilde{\mathscr{E}}=\left(u^{\widetilde{A}}, u^{B}, e^{\widetilde{A}}, e^{B}, \pi\right)$ which differ only in the utility function of the first agent. More precisely, in $\widetilde{\mathscr{E}}$ agent $A$ is replaced by agent $\widetilde{A}$ with the same endowment $e^{\widetilde{A}}=e^{A}$ but with utility function $u^{\widetilde{A}}=k \circ u^{A}$, where $k$ is a strictly increasing and concave, twice differentiable function; hence, $k^{\prime}>0$ and $k^{\prime \prime}<0$ on the interior of the domain of $k$. Thus, $\widetilde{A}$ is more risk averse than $A$ according to the familiar Arrow-Pratt measure of risk aversion. The main purpose of this chapter is to investigate the effect of increased risk aversion on the utility of agent $B$ through its effect on equilibrium. A direct comparison between the equilibrium utilities of $A$ and $\widetilde{A}$ is meaningless since the utility functions of these agents are not comparable with respect to magnitude.

In order to keep the presentation as simple as possible, we assume that the equilibria of $\mathscr{E}$ and $\widetilde{\mathscr{E}}$ are unique and interior. The uniqueness assumption renders it possible to make unambiguous comparisons between agent $B$ 's utility levels in equilibrium. ${ }^{8}$

The following lemma compares the offer curves of $A$ and $\widetilde{A}$ and therefore plays a crucial role in the proof of our main result.

Lemma 4. Let $p=\left(p_{1}, p_{2}\right)$ be a vector of positive prices and let $x=x(p)$ and $\widetilde{x}=\widetilde{x}(p)$ be the corresponding interior demands of A and $\widetilde{A}$, respectively.

(i) If $x_{1}=x_{2}$, then $x_{1}=\widetilde{x}_{1}$ and $x_{2}=\widetilde{x}_{2}$.

(ii) If $x_{1}>x_{2}$, then $\widetilde{x}_{1}>\widetilde{x}_{2}, \widetilde{x}_{1}<x_{1}$ and $\widetilde{x}_{2}>x_{2}$.

(iii) If $x_{1}<x_{2}$, then $\widetilde{x}_{1}<\widetilde{x}_{2}, \widetilde{x}_{1}>x_{1}$ and $\widetilde{x}_{2}<x_{2}$.

Geometrically, this lemma can be summarized as follows: (i) the point on the offer curve of $A$ and the certainty line is also the point on the offer curve of $\widetilde{A}$ at the same prices; (ii) for a point on the offer curve of $A$ below the certainty line, the corresponding (that is, at the same prices) point on the offer curve of $\widetilde{A}$ lies also below the certainty line but closer to the certainty line, in the 'North-West' quadrant with respect to the original point; (ii) for a point on the offer curve

\footnotetext{
${ }^{8}$ Our conditions do not guarantee uniqueness. Familiar conditions for this like the gross substitutes condition (e.g., Takayama [34]) are not satisfied. Our results below can be adapted to the case where the equilibria are not unique (this can occur) but then we would have to keep track of the equilibria that we are comparing.
} 
of $A$ above the certainty line, the corresponding (that is, at the same prices) point on the offer curve of $\widetilde{A}$ lies also above the certainty line but closer to the certainty line, in the 'South-East' quadrant with respect to the original point. This confirms our intuition with regard to the fact that $\widetilde{A}$ is more risk averse than $A$.

Proof of Lemma 4. By (3.2) we have

$$
\frac{p_{1}}{p_{2}}=\frac{\pi u^{\prime}\left(x_{1}\right)}{(1-\pi) u^{\prime}\left(x_{2}\right)}=\frac{\pi k^{\prime}\left(u\left(\widetilde{x}_{1}\right)\right) u^{\prime}\left(\widetilde{x}_{1}\right)}{(1-\pi) k^{\prime}\left(u\left(\widetilde{x}_{2}\right)\right) u^{\prime}\left(\widetilde{x}_{2}\right)}
$$

To show (i), let $x_{1}=x_{2}$. Then (3.5) implies $\widetilde{x}_{1}=\widetilde{x}_{2}$ and the budget restriction in (3.1) then implies $x_{1}=x_{2}=\widetilde{x}_{1}=\widetilde{x}_{2}$. To show (ii), let $x_{1}>x_{2}$. Then (3.5) implies $\widetilde{x}_{1}>\widetilde{x}_{2}$ and, moreover,

$$
\frac{u^{\prime}\left(x_{1}\right)}{u^{\prime}\left(x_{2}\right)}<\frac{u^{\prime}\left(\widetilde{x}_{1}\right)}{u^{\prime}\left(\widetilde{x}_{2}\right)}
$$

by concavity of $k$. Together with the budget restriction $p_{1} x_{1}+p_{2} x_{2}=p_{1} \tilde{x}_{1}+$ $p_{2} \tilde{x}_{2}\left(=p_{1} e_{1}^{A}+p_{2} e_{2}^{A}\right)$, this implies $x_{1}>\widetilde{x}_{1}$ and $x_{2}<\widetilde{x}_{2}$. The proof of (iii) is analogous.

Let $V$ denote the indirect utility function associated with (3.1), i.e., $V(p)=$ $\pi u\left(x_{1}(p)\right)+(1-\pi) u\left(x_{2}(p)\right)$ for every vector of positive prices $p=\left(p_{1}, p_{2}\right)$. Then the partial derivatives of $V$ exist. The following lemma is concerned with the sign of these derivatives and establishes when utility increases or decreases along the offer curve.

Lemma 5. Consider an agent in $\mathscr{E}$ with utility function $u$, endowment $e$ and indirect utility function $V$. Let $x=x(p)$ be an interior demand. Then:

(i) If $x_{1}<e_{1}$, then $\frac{d V}{d p_{1}}>0$.

(ii) If $x_{1}=e_{1}$, then $\frac{d V}{d p_{1}}=0$.

(iii) If $x_{1}>e_{1}$, then $\frac{d V}{d p_{1}}<0$.

Proof. Without loss of generality set $p_{2}=1$. Then

$$
\frac{d V}{d p_{1}}=\pi u^{\prime}\left(x_{1}\right) \frac{d x_{1}}{d p_{1}}+(1-\pi) u^{\prime}\left(x_{2}\right)\left(e_{1}-x_{1}-p_{1} \frac{d x_{1}}{d p_{1}}\right) .
$$

By (3.2), this implies

$$
\frac{d V}{d p_{1}}=(1-\pi) u^{\prime}\left(x_{2}\right)\left(e_{1}-x_{1}\right)
$$

This implies (i)-(iii). 
The next result shows how prices change in the equilibrium of $\widetilde{\mathscr{E}}$ compared to the equilibrium of $\mathscr{E}$.

Lemma 6. Let $c=\left(c_{1}^{A}, c_{2}^{A}, c_{1}^{B}, c_{2}^{B}\right)$ and $d=\left(d_{1}^{\widetilde{A}}, d_{2}^{\widetilde{A}}, d_{1}^{B}, d_{2}^{B}\right)$ be the unique, interior equilibrium allocations and $q$ and $\widetilde{q}$ the corresponding equilibrium prices of $\mathscr{E}$ and $\widetilde{\mathscr{E}}$, respectively, with $q_{2}=\widetilde{q}_{2}=1$. Then:

(i) If $c_{1}^{A}>c_{2}^{A}$ then $\widetilde{q}_{1} \leq q_{1}$.

(ii) If $c_{1}^{A}<c_{2}^{A}$ then $\widetilde{q}_{1} \geq q_{1}$.

Typical examples of cases (i) and (ii) in Lemma 6 are drawn in Figure 3.2. The result is quite intuitive. In case (i), by comonotonicity (cf. Lemma 3), $w_{1}>$ $w_{2}$ and increased risk aversion increases the relative price of the good in the low aggregate state. Case (ii) has an analogous explanation.

Proof of Lemma 6. We only prove (i), case (ii) is analogous. Let $c_{1}^{A}>c_{2}^{A}$ and $c_{1}^{A}>e_{1}^{A}$ (the case where $c_{1}^{A} \leq e_{1}^{A}$ is again analogous). We normalize all price vectors by setting the state 2 price equal to 1 , and write $x\left(p_{1}\right)$ for the demand vector $x(p)$. We use $x^{A}\left(p_{1}\right)$ and $x^{B}\left(p_{1}\right)$ to denote demand vectors of $A$ and $B$, respectively.

We start with a useful observation. Let $p_{1}^{A}$ be the price such that $e^{A}=$ $x^{A}\left(p_{1}^{A}\right)$. Lemma 1(i) and $c_{1}^{A}>e_{1}^{A}$ imply $q_{1}<p_{1}^{A}$. Define $C$ to be the set

$$
\begin{array}{r}
C=\bigcup_{q_{1} \leq p_{1} \leq p_{1}^{A}}\left\{\left(x_{1}^{A}, x_{2}^{A}, x_{1}^{B}, x_{2}^{B}\right) \in \mathbb{R}^{4} \mid e_{1}^{A} \leq x_{1}^{A} \leq x_{1}^{A}\left(p_{1}\right),\right. \\
\left.p_{1} x_{1}^{A}+x_{2}^{A}=p_{1} e_{1}+e_{2}, x^{A}+x^{B}=e^{1}+e^{2}\right\} .
\end{array}
$$

In words, $C$ is the union of all line segments with as one endpoint the endowment point and as other endpoint a point on the offer curve of $A$ between $e$ and $c$ : observe that $e_{1}^{A}<x_{1}^{A}\left(p_{1}\right)<c_{1}^{A}$ for every $q_{1}<p_{1}<p_{1}^{A}$ by Lemma 1. Also, $C$ is a subset of feasible allocations since, by Lemma 2(i), every element of $C$ is below the budget line through $e$ and $c$, and by the previous observation, has first coordinate between $e_{1}^{A}$ and $c_{1}^{A}$. It can be verified that $C$ is a closed set with as boundary the union of the feasible allocations on the offer curve of $A$ below the budget line through $e$ and $c$ in the Edgeworth box, and the line segment between $e$ and $c$. In fact, $C$ is the set between the offer curve of $A$ and the line segment connecting $e$ and $c$, but our definition of $C$ turns out to be useful below. 


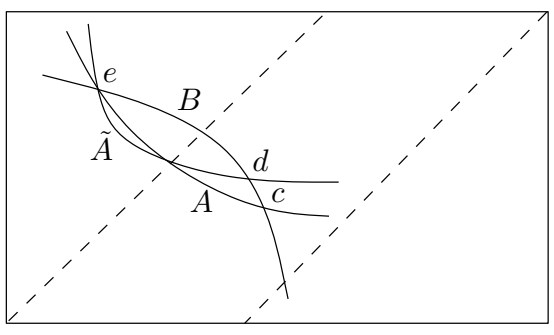

$(i), c_{1}^{A}>e_{1}^{A}$

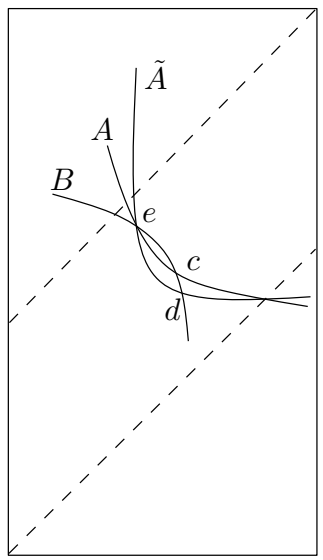

$(i i), c_{1}^{A}>e_{1}^{A}$

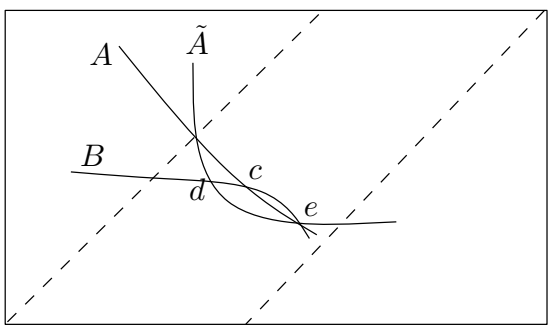

$(i), c_{1}^{A} \leq e_{1}^{A}$

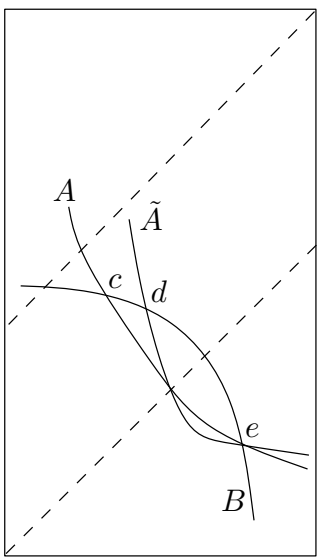

$(i i), c_{1}^{A} \leq e_{1}^{A}$

Figure 3.2: This figure sketches some instances of the cases in Lemma 6. In each case an Edgeworth box is drawn with the origin for $A$ and $\widetilde{A}$ at the lower left point and the origin for $B$ at the upper right point. Also the agents' certainty lines are drawn. In all four panels, three offer curves are drawn, and the equilibrium allocations $c$ and $d$ are the points of intersection. The two upper panels are examples of case (i) in Lemma 6, while the two lower panels correspond to case (ii) in Lemma 6 . The numbering of the panels refers to the corresponding cases in Theorem 1. 
We claim that $e$ and $c$ are the only feasible allocations in $C$ on the offer curve of $B$. Suppose not, and let $y=\left(y^{A}, y^{B}\right)$ be another feasible allocation in $C$ on the offer curve of $B$. Let $p_{1}^{B}$ such that $x^{B}\left(p_{1}^{B}\right)=e^{B}$. By Lemma $1, p_{1}^{B}<q_{1}$. Since $y \in C$ and, thus, $y_{1}^{B}<e_{1}^{B}$ and $y_{2}^{B}>e_{2}^{B}$, Lemma 2 and Lemma 1 imply that $y^{B}=x^{B}\left(p_{1}^{y}\right)$ for some price $p_{1}^{y}>q_{1}$. By Lemma 1(ii), it follows in particular that $x_{2}^{B}\left(p_{1}\right)>c_{2}^{B}>e_{2}^{B}$ for all $p_{1} \geq p_{1}^{y}$. Choose $p_{1}^{z}>\max \left\{p_{1}^{y}, p_{1}^{A}\right\}$, then the feasible allocation $z$ with $z^{B}=x^{B}\left(p_{1}^{z}\right)$ is not in $C$. Since $C$ is a closed set and the offer curve of $B$ a continuous curve, this curve must intersect the boundary of $C$ at a point $v$ for some price between $p_{1}^{y}$ and $p_{1}^{z}$. Since $v$ cannot be a point on the line segment connecting $e$ and $c$, it must be a point on the offer curve of $A$ unequal to $e$ or $c$. But then, $v$ is an equilibrium allocation, contradicting our assumption that equilibrium is unique.

Now suppose, contrary to what we wish to prove, that $\widetilde{q}_{1}>q_{1}$. Lemma 3 and $c_{1}^{A}>c_{2}^{A}$ imply $w_{1}>w_{2}$, and therefore, by Lemma 3 again, $d_{1}^{\widetilde{A}}>d_{2}^{\widetilde{A}}$. Since $d$ is on the offer curve of $B, \widetilde{q}_{1}>q_{1}>p_{1}^{B}$ and Lemma 1(ii) imply $d_{2}^{B}>e_{2}^{B}$ and, thus, $d_{1}^{B}<e_{1}^{B}$. Consider the feasible allocation $x$ with $x^{A}=x^{A}\left(\widetilde{q}_{1}\right)$, which is located on the offer curve of $A$ and the budget line through $e$ and $d$. If $x$ is on the same side of $d$ as $e$ is, i.e., if $x_{1}^{A} \leq d_{1}^{\widetilde{A}}$, then consider two cases. If $x_{1}^{A}>x_{1}^{B}$, then, by Lemma 4(ii), $d^{\widetilde{A}}$ has first coordinate smaller than $x_{1}^{A}$, a contradiction. If $x_{1}^{A} \leq x_{1}^{B}$, then by Lemma 4 (i) or (iii), $d_{1}^{\widetilde{A}} \leq d_{2}^{\widetilde{A}}$, again a contradiction. Hence, we must have $x_{1}^{A}>d_{1}^{\widetilde{A}}$. Then $\widetilde{q}_{1} \leq p_{1}^{A}$ since otherwise $x_{1}^{A}<e_{1}^{A}<d_{1}^{A}$ by Lemma 1(i), a contradiction. Hence $q_{1}<\widetilde{q}_{1} \leq p_{1}^{A}$, which implies that $d$ is in the set $C$, contradicting our claim above.

Thus, we must have $\widetilde{q}_{1} \leq q_{1}$.

Let $q$ and $\widetilde{q}$ be the equilibrium prices in $\mathscr{E}$ and $\widetilde{\mathscr{E}}$, respectively. Let $V^{B}$ denote the indirect utility function of agent $B$. We say that risk aversion (of the opponent) benefits agent $B$ if $V^{B}(\widetilde{q}) \geq V^{B}(q)$ and risk aversion (of the opponent) hurts agent $B$ if $V^{B}(\widetilde{q}) \leq V^{B}(q)$. The following theorem answers the question when risk aversion benefits or hurts agent $B$.

Theorem 1. Let $c=\left(c_{1}^{A}, c_{2}^{A}, c_{1}^{B}, c_{2}^{B}\right)$ and $d=\left(d_{1}^{\widetilde{A}}, d_{2}^{\widetilde{A}}, d_{1}^{B}, d_{2}^{B}\right)$ be the unique, interior equilibrium allocations and $q$ and $\widetilde{q}$ the corresponding equilibrium prices of $\mathscr{E}$ and $\widetilde{\mathscr{E}}$. Then:

(i) If $w_{1}=w_{2}$, then $q=\widetilde{q}$ and $c=d$, so $V^{B}(q)=V^{B}(\widetilde{q})$.

(ii) If $w_{1}>w_{2}$, then 
(ii.a) if $e_{1}^{A} \leq e_{2}^{A}$, then risk aversion hurts agent $B$.

(ii.b) if $e_{1}^{A}>e_{2}^{A}$ and $e_{1}^{A} \geq c_{1}^{A}$, then risk aversion benefits agent $B$.

(ii.c) if $e_{1}^{A}>e_{2}^{A}, e_{1}^{A}<c_{1}^{A}$, and $e_{1}^{B} \geq d_{1}^{B}$, then risk aversion hurts agent $B$.

(iii) If $w_{1}<w_{2}$, then

(iii.a) if $e_{1}^{A} \geq e_{2}^{A}$, then risk aversion hurts agent $B$.

(iii.b) if $e_{1}^{A}<e_{2}^{A}$ and $e_{1}^{A} \leq c_{1}^{A}$, then risk aversion benefits agent $B$.

(iii.c) if $e_{1}^{A}<e_{2}^{A}, e_{1}^{A}>c_{1}^{A}$, and $e_{1}^{B} \leq d_{1}^{B}$, then risk aversion hurts agent $B$.

Proof. Case (i) follows directly from Lemma 3 and Lemma 4(i).

For case (ii.a), it follows from Lemma 3 and Lemma 6(i) that $\widetilde{q}_{1} \leq q_{1}$. From Lemma 3 again and from Lemma $5(\mathrm{i}), V^{B}(\widetilde{q}) \leq V^{B}(q)$, so risk aversion hurts $B$.

For case (ii.b), by Lemma 3 and Lemma 6(i), $\widetilde{q}_{1} \leq q_{1}$. By Lemma 5(ii),(iii), $V^{B}(\widetilde{q}) \geq V^{B}(q)$, so risk aversion benefits $B$.

For case (ii.c), by Lemma 3 and Lemma $6(\mathrm{i}), \widetilde{q}_{1} \leq q_{1}$. By Lemma $5(\mathrm{i}), V^{B}(\widetilde{q}) \leq$ $V^{B}(q)$, so risk aversion hurts $B$.

The analogous proofs for cases (iii.a)-(iii.c) are omitted.

Theorem 1 can be summarized in a non-technical way as follows. This summary also provides a good intuition for the results. If agent $A$ is replaced by a more risk averse agent $\widetilde{A}$, then the equilibrium allocation moves closer to agent $A$ 's (or $\widetilde{A}$ 's) certainty line, as is intuitive. This movement takes place along agent $B$ 's offer curve. If the equilibrium allocation moves away from the endowment point $e$, then this is beneficial to agent $B$, as follows from Lemma 5 . If it moves towards $e$, then this hurts agent $B$. The latter observation also explains why Theorem 1 is not exhaustive. Cases (ii.c) and (iii.c), where the equilibrium allocation indeed moves towards the endowment point, are restricted by the condition that the equilibrium allocation should not actually cross the endowment point, since in that case, agent $B$ 's utility starts to increase again, so that the final effect is ambiguous. Example 7 below shows that this can indeed happen.

Cases (ii.b) and (iii.b) of Theorem 1 are illustrated in the upper right and lower left panels of Figure 3.2, respectively. In both cases the movement from $c$ to $d$ along the offer curve of $B$ is away from the endowment point $e$ : this benefits agent $B$. Cases (ii.a) and (iii.a) of Theorem 1 are illustrated in the upper left and lower right panels of Figure 3.2, respectively. In both cases the movement 
from $c$ to $d$ along the offer curve of $B$ is towards endowment point $e$ : this hurts agent $B$.

We will illustrate two of these cases with an example, phrased in terms of an insurer and an insured.

Example 6. Let agent $A$ possess wealth 2 . There is a $50 \%$ chance that $A$ suffers a loss of 1 , against which he wishes to insure himself. The insurer is agent $B$, and we assume that $B$ has wealth 2 as well. The von Neumann-Morgenstern utility functions of both agents are $t \mapsto \ln (t+2)$. Hence, this situation can be modeled by a two-agent two-state economy where $\pi=\frac{1}{2}, e^{A}=(2,1)$, and $e^{B}=(2,2)$. So state 2 is the state in which agent $A$ suffers a loss of 1 . The equilibrium allocation in this economy is $c^{A}=\left(\frac{36}{21}, \frac{35}{28}\right)$ and $c^{B}=\left(\frac{16}{7}, \frac{7}{4}\right)$, while the equilibrium prices are $\left(1, \frac{8}{7}\right)$. Observe that $\frac{8}{7}$, the price of state 2 , is the unit premium that $A$ pays $B$ for the insurance. By Theorem 1, case (ii.b), it follows that if the insured would become more risk averse, then this is beneficial to the insurer. Suppose, indeed, that agent $A$ is replaced by a more risk averse agent $\widetilde{A}$, with utility function $t \mapsto \ln (t+1)$. Then the equilibrium allocation changes to $d^{\widetilde{A}}=\left(\frac{35}{21}, \frac{36}{28}\right)$, $d^{B}=\left(\frac{7}{3}, \frac{12}{7}\right)$ and the prices to $\left(1, \frac{7}{6}\right)$. It is easy to check that $B$ 's utility has indeed increased. Compared to agent $A$ 's equilibrium allocation, agent $\widetilde{A}$ 's allocation has shifted to the North-West in the Edgeworth box, closer to the certainly line. Agent $\widetilde{A}$ demands more insurance (state 2 good), the price of state 2 goes up, and agent $B$, who sells the insurance, benefits. This is quite intuitive, but we should nevertheless be careful in expressing such an intuition, as the following will show.

Suppose now $e^{B}=\left(\frac{1}{2}, 2\right)$. This could be, for instance, because in state 1 (the good state for the insured) the insurer has to pay claims to another insured party. For this case, Theorem 1, case (iii.a), implies that the insurer is hurt if the insured becomes more risk averse. One can compute that with agent $A$ the equilibrium allocation is $c^{A}=\left(\frac{117}{84}, \frac{473}{286}\right), c^{B}=\left(\frac{31}{28}, \frac{35}{26}\right)$, and the equilibrium prices are $\left(1, \frac{13}{14}\right)$. With agent $\widetilde{A}$ we have: $d^{\widetilde{A}}=\left(\frac{119}{48}, \frac{468}{286}\right), d^{B}=\left(\frac{13}{12}, \frac{15}{11}\right)$, and equilibrium prices $\left(1, \frac{11}{12}\right)$. It is straightforward to check that, indeed, agent $B$ is now hurt. Compared to agent $A$ 's equilibrium allocation, agent $\widetilde{A}$ 's allocation has shifted to the South-East in the Edgeworth box, closer to the certainly line. Agent $\widetilde{A}$ demands less insurance (state 2 good), the price of state 2 goes down, and agent $B$, who sells the insurance, is hurt. Due to the relative scarcity of good 1, a more risk averse insured buys, in fact, less insurance in order to obtain more equal amounts in both states. 
The next example exhibits one of the cases, not covered by Theorem 1, where the effect of increased risk aversion is ambiguous.

Example 7. Consider a two-agent, two-state economy in which both states are equally likely. Agent $A$ has utility function $t \mapsto \ln (2+t)$ and endowment $e^{A}=\left(\frac{11}{10}, \frac{13}{20}\right)$. Agent $B$ has utility function $t \mapsto \ln (1+t)$ and endowment $e^{B}=$ $\left(\frac{19}{10}, \frac{27}{20}\right)$. This economy has equilibrium allocation $c$ with $c^{B}=\left(\frac{57}{50}, \frac{83}{60}\right)$. Suppose that agent $A$ is replaced by a more risk averse agent $\widetilde{A}$ with utility function $t \mapsto \ln \left(\frac{1}{10}+t\right)$. The equilibrium allocation $d$ which results from this replacement in agents has $d^{B}=\left(\frac{497}{248}, \frac{417}{328}\right)$. Clearly, $c_{1}^{B}<e_{1}^{B}<d_{1}^{B}$ and agent $B$ 's expected utility at $d(\approx 0.9602)$ is higher than at $c(\approx 0.9597)$. Theorem 1 does not apply here, since the last condition in case (ii.c) is not fulfilled: by going from $c$ to $d$ along $B$ 's offer curve, endowment point $e$ is crossed.

Suppose now that agent $A$ is replaced by agent $\bar{A}$ with utility function $t \mapsto$ $\ln (1+t)$. Agent $\bar{A}$ is more risk averse than $A$, but less so than $\widetilde{A}$. The new equilibrium allocation is the point $\bar{d}$ with $\bar{d}^{B}=\left(\frac{307}{160}, \frac{267}{200}\right)$. This equilibrium yields agent $B$ an expected utility equal to approximately 0.9596 , which is lower than the expected utility he receives in allocation $c$. Clearly $c_{1}^{B}<e_{1}^{B}<\bar{d}_{1}^{B}$ which means again that Theorem 1 does not apply.

\subsection{Concluding Remarks}

The model and results in this chapter are limited to two agents and two states of the world. This is restrictive but nevertheless applies to situations where we can distinguish between roughly two types of agents and two sets of states. Extending the model and results to more than two agents and/or more than two states may not be straightforward, due to the many different possible configurations of endowments and equilibrium allocations. A potentially useful tool is provided by the concept of a representative agent, as proposed by Wilson (1968). Increased risk aversion of one of the agents may be reflected by increased risk aversion of the representative agent which, in turn, affects the equilibrium prices (similar to Lemma 6 above - see also, e.g., Gollier [18] Ch. 22). It is not clear, however, whether conclusions about the welfare of individual agents can be drawn easily from this. When there are only two states, as presented in this chapter, price changes are unidirectional, but in case of more than two states prices might change in different directions. Similarly, a complication in case of more than two agents is that agents' utilities may be affected 
in different directions if one agent is replaced by a more risk averse agent. In fact, the following example reveals such a case.

Example 8. Consider a three agent, two state economy in which every agent $A, B$, and $C$ has the same utility function $t \mapsto \ln (1+t)$, and the endowments are $e^{A}=(0,3), e^{B}=(3,0)$, and $e^{C}=(1,2) .{ }^{9}$ We assume that each state is equally likely. It can be verified that this economy has a unique equilibrium allocation, namely $x^{A}=\left(\frac{20}{16}, \frac{22}{14}\right), x^{B}=\left(\frac{23}{16}, \frac{25}{14}\right)$, and $x^{C}=\left(\frac{21}{16}, \frac{23}{14}\right)$. Now suppose that agent $A$ is replaced by a more risk averse agent $\widetilde{A}$ with utility function $t \mapsto \ln \left(\frac{1}{10}+t\right)$. In this new economy with the more risk averse agent $\widetilde{A}$ the (unique) equilibrium allocation is $y^{\widetilde{A}}=\left(\frac{981}{710}, \frac{92}{61}\right), y^{B}=\left(\frac{103}{42}, \frac{223}{122}\right)$, and $y^{C}=\left(\frac{183}{142}, \frac{203}{122}\right)$. It is straightforward to verify that agent $B$ benefits from agent $A$ being replaced by the more risk averse agent $\widetilde{A}$, while agent $C$ is hurt by it.

For particular choices of utility functions (e.g., linear risk tolerance functions) it may be possible to obtain some more general results, but this is left for further research.

In spite of the fact that risk aversion has been extensively studied in ArrowDebreu models - see, e.g., recently Hara et al. [19] - to the best of our knowledge a comparative study with respect to risk aversion like the one in this chapter, has not explicitly been carried out before. The impact of increased risk aversion has been studied before in other fields, e.g. in the game theory. See, for instance, Kihlstrom et al. [22] and Köbberling and Peters [23] for bargaining games, and Berden and Peters [7] for two-person noncooperative games.

\footnotetext{
${ }^{9}$ Strictly speaking, we excluded zero endowments, but the example still works if we assume small positive numbers instead of the zeros.
} 



\section{The Role of Individual Intertemporal Transfers in Dynamic TU-Games}

\subsection{Introduction}

A cooperative game with transferable utility, denoted TU-game, is characterized by a function that gives each coalition of players a nonnegative worth. It describes a one-time event. In reality, many events occur more than once, e.g. contract negotiations. This can be captured by a dynamic TU-game, which consists of a sequence of TU-games and intertemporal utility functions for all players. The convention is that a solution is applied to every TU-game in the sequence separately, thereby generating a payoff distribution for every period. Each player can evaluate his resulting stream of payoffs by the intertemporal utility function. A limitation of this static framework is that it does not capture dynamic features, such as the possibility of borrowing and saving or history dependent games and/or solutions, and thus extra tools are necessary. The first mentioned feature is briefly discussed in a paper by Kranich et al. [24], in which theoretical tools are developed to analyze dynamic TU-games under the assumption of exogenously specified TU-games. Filar and Petrosjan [16], on the other hand, consider endogenously determined TU-games that depend on payoffs obtained in earlier periods. Their focus is on solutions which are time consistent to the TU-games.

In this chapter ${ }^{1}$ we implement the idea of borrowing and saving, by allowing players to transfer payoffs across periods. In other words, each player is faced with individual intertemporal transfer decisions that affect the sequence

${ }^{1}$ This chapter is based on Berden [6]. 
of TU-games and as a result affect the stream of payoffs to every player.

We consider two different transfer systems. In the first transfer system $(k=$ $1)$ an individual intertemporal transfer only influences the individual worths of TU-games within a dynamic TU-game. In contrast, in the second system $(k=$ 2) an individual intertemporal transfer also influences all worths of coalitions over time which contain the individual. We hereby assume that the change of a coalition's worth equals the sum of changes of the individual worths of players contained in the coalition. To ensure that the worths of coalitions remain nonnegative under the second transfer system, only dynamic TU-games with weakly superadditive TU-games are considered. The strategic possibilities which result from individual intertemporal transfers are modeled by a noncooperative game, which depends on the underlying dynamic TU-game, a solution concept and a transfer system.

We use additive and restrictively additive solution concepts. The Shapley value (Shapley [33]) is an example of a (restrictively) additive solution. The nucleolus (Schmeidler [32]) and prenucleolus are restrictively additive solutions. We focus on the case in which a given set of players play a finite sequence of exogenously specified TU-games. Time is considered to be discrete. ${ }^{2}$ Intertemporal utility functions that value payoff in time periods closer to the present more than the same payoff in time periods further away from the present are considered.

In Theorem 5 we prove that a Nash equilibrium exists in our noncooperative game under both transfer systems $k \in\{1,2\}$ if the solution is continuous and transfer- $k$-concave and if the intertemporal utility functions of the dynamic TU-game are continuous, increasing and quasi-concave. These assumptions ensure that the utility functions of the noncooperative game are continuous and quasi-concave, which are sufficient conditions under which Debreu [15] proved existence of a Nash equilibrium in a noncooperative game. Example 4 illustrates that a Nash equilibrium does not have to exist in case the nucleolus is applied to a dynamic TU-game where payoffs are transferred according to the first transfer system. This is consistent with the fact that the nucleolus is not transfer-1-concave, i.e. the nucleolus is not concave under the first transfer system. Therefore not all utility functions of the associated noncooperative game are quasi-concave. Hence, transfer- $k$-concavity of the solution cannot be

\footnotetext{
${ }^{2}$ Similar assumptions are made in Kranich et al. [25] in which different core concepts for dynamic games with transferable utility are considered.
} 
dropped from the theorem. Additionally, we show that a Nash equilibrium in dominant strategies exists if the intertemporal utility functions of the dynamic TU-game value present payoff more than the same amount of future payoff and one of two conditions are met: (i) payoffs are transferred according to the first transfer system, the solution is additive and some other specific conditions on the solution hold or (ii) payoffs are transferred according to the second transfer system, the dynamic game only consists of weakly superadditive TU-games, the solution is restrictively additive and another specific condition on the solution hold. In this special type of Nash equilibrium players transfer as much payoff as possible to the present. We show that this Nash equilibrium is Pareto optimal, under particular conditions.

The organization of Chapter 4 is as follows. Section 4.2 presents main definitions. Section 4.3 presents the strategic model and the main results. Section 4.4 is devoted to Pareto optimality. Section 4.5 concludes.

\subsection{Preliminaries}

A TU-game consists of a pair $(N, v)$ where $N:=\{1, \ldots, n\}$ denotes the set of players and $v: 2^{N} \rightarrow \mathbb{R}$ is a characteristic function which assigns to each coalition $S \subseteq N$ the worth $v(S)$ and $v(\varnothing)=0$. In the remainder we omit $N$ from notation and simply write $v$. The set of all TU-games with player set $N$ is denoted by $\mathscr{G}^{N}$. By $\mathscr{G}_{+}^{N}$ we denote the set of all TU-games with $v(S) \geq 0$ for all $S \subseteq N$. A game $v \in \mathscr{G}^{N}$ is additive if $v(S \cup T)=v(S)+v(T)$ for all disjoint $S, T \subseteq N$. Hence, the additive game $v \in \mathscr{G}^{N}$ is generated by the vector $(\nu(1), \ldots, v(n)) \in \mathbb{R}^{N}$. A game $v \in \mathscr{G}^{N}$ is weakly superadditive in case $v(S) \geq \sum_{i \in S} v(i)$ for all $S \subseteq N$. The zerogame is denoted by $z \in \mathscr{G}^{N}$.

Definition 1. Let $T \in \mathbb{N}$. A dynamic $T U$-game $\Gamma$ is a pair $(\mathbf{v}, \mathbf{u})$ where

(i) $\mathbf{v}=\left(v^{1}, \ldots, v^{T}\right) \in\left(\mathscr{G}_{+}^{N}\right)^{T}$.

(ii) $\mathbf{u}=\left(u_{1}, \ldots, u_{n}\right)$ where, for each $i \in N, u_{i}: \mathbb{R}^{T} \rightarrow \mathbb{R}$.

In a dynamic TU-game we restrict ourselves to nonnegative TU-games in order to have a natural lower bound to payoff transfers between periods, to be introduced later. Under certain circumstances we will even add the restriction that TU-games have to be weakly superadditive. 
The function $u_{i}$ is an intertemporal utility function which assigns to every payoff stream $\mathbf{x} \in \mathbb{R}^{T}$ of player $i$ utility level $u_{i}(\mathbf{x})$. If for all $\mathbf{x}, \mathbf{y} \in \mathbb{R}^{T}$ with $\mathbf{x}>\mathbf{y}^{3}$ it holds that $u_{i}(\mathbf{x}) \geq u_{i}(\mathbf{y})$, then $u_{i}$ is increasing. If in this situation $u_{i}(\mathbf{x})>u_{i}(\mathbf{y})$, then $u_{i}$ is strictly increasing. The function $u_{i}$ is quasi-concave if for all $\mathbf{x}, \mathbf{y} \in \mathbb{R}^{T}$ and $\lambda \in(0,1)$ it holds that $u_{i}(\lambda \mathbf{x}+(1-\lambda) \mathbf{y}) \geq \min \left\{u_{i}(\mathbf{x}), u_{i}(\mathbf{y})\right\}$.

In order to define a special class of intertemporal utility functions, the vector $\mathbf{e}^{t} \in \mathbb{R}^{T}$ is defined which has a 1 for the element on place $t$ and zeros for all other elements.

Definition 2. The class $V$ [class $\bar{V}$ ] contains intertemporal utility functions $u$ : $\mathbb{R}^{T} \rightarrow \mathbb{R}$ with the property that for all $\mathbf{x}, \mathbf{y} \in \mathbb{R}^{T}$, all $t, t^{\prime} \in\{1, \ldots, T\}$ and $\epsilon>0$ it holds that if $t<t^{\prime}$ and $\mathbf{x}=\mathbf{y}+\epsilon \mathbf{e}^{t}-\epsilon \mathbf{e}^{t^{\prime}}$, then $u(\mathbf{x}) \geq u(\mathbf{y})[u(\mathbf{x})>u(\mathbf{y})]$.

By definition, $\bar{V} \subset V$. Discounting a stream of payoffs is contained in the class $V$. The next lemma characterizes differentiable utility functions in $V$.

Lemma 7. For all utility functions $u: \mathbb{R}^{T} \rightarrow \mathbb{R}$ for which the partial derivatives exist and are continuous and $u_{t}^{\prime}(\mathbf{x}) \geq 0{ }^{4}$ for all $t$ and all $\mathbf{x} \in \mathbb{R}^{T}$, we have $u \in V$ if and only if $u_{t}^{\prime}(\mathbf{x}) \geq u_{t^{\prime}}^{\prime}(\mathbf{x})$ for every $\mathbf{x} \in \mathbb{R}^{T}$ and all $t<t^{\prime}$.

Proof. Let $t<t^{\prime}$ and $\mathbf{y}_{\eta}:=\mathbf{y}+\eta \mathbf{e}^{t}-\eta \mathbf{e}^{t^{\prime}}$ for all $\mathbf{y} \in \mathbb{R}^{T}$ and $\eta>0$. Let $\epsilon>0$ and define the function $f:[0, \epsilon] \rightarrow \mathbb{R}$ by $f: \eta \mapsto u\left(\mathbf{y}_{\eta}\right)$. Then, using the chain rule,

$$
\begin{aligned}
u\left(\mathbf{y}_{\epsilon}\right)-u\left(\mathbf{y}_{0}\right) & =f(\epsilon)-f(0) \\
& =\int_{0}^{\epsilon} f^{\prime}(\eta) d \eta=\int_{0}^{\epsilon}\left[\frac{\partial u\left(\mathbf{y}_{\eta}\right)}{\partial \eta}\right] d \eta \\
& =\int_{0}^{\epsilon}\left[\sum_{s=1}^{T} u_{s}^{\prime}\left(\mathbf{y}_{\eta}\right) \cdot \frac{\partial y_{\eta}^{s}}{\partial \eta}\right] d \eta \\
& =\int_{0}^{\epsilon}\left[u_{t}^{\prime}\left(\mathbf{y}_{\eta}\right) \cdot 1+u_{t^{\prime}}^{\prime}\left(\mathbf{y}_{\eta}\right) \cdot-1\right] d \eta .
\end{aligned}
$$

(i) To prove the 'only if' part, assume $u \in V$ and let $t<t^{\prime}$. Suppose contrary to what has to be proven that $u_{t}^{\prime}(\mathbf{z})<u_{t^{\prime}}^{\prime}(\mathbf{z})$ for some $\mathbf{z} \in \mathbb{R}^{T}$. Define $\mathbf{B}_{\theta}(\mathbf{z})$ as the $\theta$-neighborhood of the vector $\mathbf{z}$ for $\theta>0$. Because of continuity of the partial derivatives, there exists a $\theta>0$ such that $u_{t}^{\prime}\left(\mathbf{z}^{\prime}\right)<u_{t^{\prime}}^{\prime}\left(\mathbf{z}^{\prime}\right)$ for all $\mathbf{z}^{\prime} \in \mathbf{B}_{\theta}(\mathbf{z})$. Choose

\footnotetext{
${ }^{3}$ The vector inequality $\mathbf{x}>\mathbf{y}$ is to be understood as $x^{t} \geq y^{t}$ for all $t \in\{1, \ldots, T\}$ and $x^{t}>y^{t}$ for at least one $t \in\{1, \ldots, T\}$.

${ }^{4}$ The notation $u_{t}^{\prime}(\mathbf{x})$ denotes the partial derivative of $u$ with respect to the $t$-th coordinate.
} 
$\mathbf{y} \in \mathbb{R}^{T}$ and $\epsilon>0$ in such a way that $\mathbf{y}_{\eta} \in \mathbf{B}_{\theta}(\mathbf{z})$ for every $\eta \in[0, \epsilon]$, then by (4.1) it follows that $u\left(\mathbf{y}_{\epsilon}\right)-u\left(\mathbf{y}_{0}\right)<0$, which is a contradiction to $u \in V$.

(ii) For the converse suppose that $u_{t}^{\prime}(\mathbf{x}) \geq u_{t^{\prime}}^{\prime}(\mathbf{x})$ for all $\mathbf{x} \in \mathbb{R}^{T}$. From (4.1) it then follows that $u\left(\mathbf{y}_{\epsilon}\right)-u\left(\mathbf{y}_{0}\right) \geq 0$, hence $u \in V$.

A value $\psi: \mathscr{G}^{N} \rightarrow \mathbb{R}^{N}$ assigns to a TU-game $v \in \mathscr{G}^{N}$ the vector $\psi(\nu)$. The value $\psi$ is additive if for all $v, w \in \mathscr{G}^{N}$ it holds that $\psi(v+w)=\psi(v)+\psi(w)$. The value $\psi$ is restrictively additive if for all $\nu, w \in \mathscr{G}^{N}$ where $w$ is an additive game generated by $\overline{\mathbf{w}}=(w(1), \ldots, w(n)) \in \mathbb{R}^{N}$ it holds that $\psi(v+w)=\psi(v)+\overline{\mathbf{w}}$. Hence, restricted additivity is a combination of the above mentioned weaker form of additivity and the condition that $\psi(w)=\overline{\mathbf{w}}$ for all additive games $w$ generated by $\overline{\mathbf{w}}=(w(1), \ldots, w(n))$. For every $i \in N, \psi_{i}$ is concave if for all $v, w \in \mathscr{G}^{N}$ and $\lambda \in(0,1)$ it holds that $\psi_{i}(\lambda v+(1-\lambda) w) \geq \lambda \psi_{i}(\nu)+(1-\lambda) \psi_{i}(w)$. For $i \in T \subseteq N, \psi_{i}$ is increasing in the worth of coalition $T$ if $\psi_{i}(v) \geq \psi_{i}\left(\nu^{\prime}\right)$ for all $v, v^{\prime} \in \mathscr{G}^{N}$ with $v(T)>v^{\prime}(T)$ and $v(S)=v^{\prime}(S)$ for all $S \neq T$. If in this situation $\psi_{i}(v)>\psi_{i}\left(v^{\prime}\right)$, then $\psi_{i}$ is strictly increasing in the worth of coalition $T$. The function $\psi_{i}$ is [strictly] decreasing in the worth of coalition $T$ if $-\psi_{i}$ is [strictly] increasing in the worth of coalition $T$.

In order to describe two transfer mechanisms later on, we define games $1_{T}, 2_{T} \in \mathscr{G}_{+}^{N}$ for $T \subset N$ as follows. The game $1_{T}$ has worth $1_{T}(S)=1$ if $S=T$ and $1_{T}(S)=0$ otherwise and the game $2_{T}$ has worths $2_{T}(S)=1$ if $T \subseteq S$ and $2_{T}(S)=0$ otherwise. Note that the game $2_{T}$ is the usual unanimity game on $T$.

The game $2_{i}{ }^{5}$ for $i \in N$ is an additive game generated by the vector $\overline{\mathbf{e}}_{i} \in$ $\mathbb{R}^{N}$ which has a 1 for the element on place $i$ and zeros for all other elements. In case $\psi$ is restrictively additive and $\psi_{i}(z)=0$ for every $i \in N$ it follows that $\psi\left(\alpha 2_{i}\right)=\alpha \overline{\mathbf{e}}_{i}$ for every $i \in N$ and all $\alpha \in \mathbb{R}$. Thus, $\psi_{i}\left(\alpha 2_{i}\right)=\alpha$ for all $i \in N$ and $\psi_{j}\left(\alpha 2_{i}\right)=0$ for all $j \in N \backslash i$ and $\alpha \in \mathbb{R}$. In the next two lemmas we look at the relation between $\psi_{i}$ and the game $1_{i}$. The results will be used for later analysis.

\section{Lemma 8.}

(i) Let $\psi$ be additive and let $\psi_{i}(z)=0$ for all $i \in N$, then $\psi_{i}\left(\alpha 1_{i}\right)=\alpha \psi_{i}\left(1_{i}\right)$ for every $i \in N$ and all $\alpha \in \mathbb{Q}$.

(ii) If, additionally to (i), $\psi_{i}$ is continuous in $v(i)$ for all $i \in N$ and all $v \in \mathscr{G}^{N}$, then $\psi_{i}\left(\alpha 1_{i}\right)=\alpha \psi_{i}\left(1_{i}\right)$ for every $i \in N$ and all $\alpha \in \mathbb{R}$.

\footnotetext{
${ }^{5}$ A coalition is written without curly braces, e.g. $i j$ is written instead of $\{i, j\}$ for $i, j \in N$.
} 
Proof. (i) Let $i \in N$ and write $\psi_{i}\left(1_{i}\right)=r$. By additivity of $\psi$ and $\psi_{i}(z)=0$ we have $\psi_{i}\left(-1_{i}\right)+\psi_{i}\left(1_{i}\right)=\psi_{i}\left(-1_{i}+1_{i}\right)=\psi_{i}(z)=0$. Hence, $\psi_{i}\left(-1_{i}\right)=-\psi_{i}\left(1_{i}\right)=-r$. By additivity it then holds that $\psi_{i}\left(k 1_{i}\right)=k \psi_{i}\left(1_{i}\right)=k r$ for $k \in \mathbb{N}$ and in a similar way $\psi_{i}\left(-k 1_{i}\right)=k \psi_{i}\left(-1_{i}\right)=-k r$. For $\frac{p}{q} \in \mathbb{Q}, \psi_{i}\left(\frac{p}{q} 1_{i}\right)=\frac{1}{q} q \psi_{i}\left(\frac{p}{q} 1_{i}\right)=\frac{1}{q} \psi_{i}\left(\frac{p}{q} q 1_{i}\right)=$ $\frac{1}{q} \psi_{i}\left(p 1_{i}\right)=\frac{1}{q} p \psi_{i}\left(1_{i}\right)=\frac{p}{q} r$. Thus, $\psi_{i}\left(\alpha 1_{i}\right)=\alpha \psi_{i}\left(1_{i}\right)$ for all $\alpha \in \mathbb{Q}$.

(ii) let $\psi_{i}$ be continuous in $v(i)$ for all $i \in N$ and all $v \in \mathscr{G}^{N}$, which implies $\psi_{i}\left(\alpha 1_{i}\right)$ is continuous in $\alpha$. Combining this with part (i) it follows that $\psi_{i}\left(\alpha 1_{i}\right)=$ $\alpha \psi_{i}\left(1_{i}\right)$ for all $\alpha \in \mathbb{R}$.

Instead of continuity in Lemma 8(ii), also monotonicity can be used to show that $\psi_{i}\left(\alpha 1_{i}\right)=\alpha \psi_{i}\left(1_{i}\right)$ for all $i \in N$ and all $\alpha \in \mathbb{R}$.

Lemma 9. Let $_{i}(z)=0$ for all $i \in N$. Let $\psi$ be additive and let $\psi_{i}$ be increasing in $v(i)$ for all $i \in N$ and all $v \in \mathscr{G}^{N}$. Then $\psi_{i}\left(\alpha 1_{i}\right)=\alpha \psi_{i}\left(1_{i}\right)$ for every $i \in N$ and all $\alpha \in \mathbb{R}$.

Proof. For $\alpha \in \mathbb{R}$, there exist $\frac{p}{q}, \frac{p^{\prime}}{q^{\prime}} \in \mathbb{Q}$ with $p, q, p^{\prime}, q^{\prime} \in \mathbb{Z}$ such that $\frac{p}{q}<\alpha<\frac{p^{\prime}}{q^{\prime}}$. Since $\psi_{i}$ is increasing in $v(i)$ for all $i \in N$ and $v \in \mathscr{G}^{N}$, it follows that $\psi_{i}\left(\alpha 1_{i}\right)$ is increasing in $\alpha$. Since $\psi_{i}(z)=0$, as a result $\psi_{i}\left(1_{i}\right) \geq 0$. From Lemma 8(i) and because $\frac{p}{q}<\alpha<\frac{p^{\prime}}{q^{\prime}}$ it follows that $\psi_{i}\left(\frac{p}{q} 1_{i}\right)=\frac{p}{q} \psi_{i}\left(1_{i}\right) \leq \psi_{i}\left(\alpha 1_{i}\right) \leq \frac{p^{\prime}}{q^{\prime}} \psi_{i}\left(1_{i}\right)=$ $\psi_{i}\left(\frac{p^{\prime}}{q^{\prime}} 1_{i}\right)$. The fractions $\frac{p}{q}, \frac{p^{\prime}}{q^{\prime}}$ can be chosen very close to $\alpha$, such that $\frac{p}{q}$ approaches $\alpha$ from below and $\frac{p^{\prime}}{q^{\prime}}$ approaches $\alpha$ from above. Thus, $\psi_{i}\left(\alpha 1_{i}\right)=$ $\alpha \psi_{i}\left(1_{i}\right)$ for all $\alpha \in \mathbb{R}$.

In the next section a solution $\psi$ is applied to dynamic TU-games. This means that $\psi$ is applied to every TU-game within the dynamic TU-game separately. Individual intertemporal transfers are introduced which influence the exogenously determined dynamic TU-game. The strategies resulting from individual intertemporal transfers are modeled in a noncooperative game.

\subsection{Individual Intertemporal Transfers}

We now assume players can make individual transfers between TU-games within a dynamic TU-game, by which they can affect their intertemporal payoffs. If a player makes an individual transfer he transfers payoff over time: this payoff transfer affects certain worths in time which can have an affect on the intertemporal payoffs of the players. No discounting is involved with these transfers, although the intertemporal utility function with which players evaluate 
their payoffs can be discounted utility. One interpretation is that each player possesses a quantity of a good which does not loose its quality over time and can be stored. The good can be transferred over time and maintain its quality, however the utility which players receive from its consumption can change, in particular decrease, over time.

We look at two different transfer systems. Under the first transfer system $(k=1)$, a player's transfer changes only his individual worths. In other words, by using a transfer, a player can redistribute his individual worths over time and thereby his stream of payoffs. Hence, a transfer by player $i$ of size $\alpha$, such that $v^{t}(i)+\alpha \geq 0$, from game $v^{t}$ to game $v^{t+1}$ results in games $v^{t}-\alpha 1_{i}$ and $v^{t+1}+\alpha 1_{i}$ were the game $1_{i}$ has worth $1_{i}(S)=1$ if $S=i$ and $1_{i}(S)=0$ otherwise. Players can evaluate transfers as follows. The utility to a player before transfer $\alpha$ is applied is equal to the intertemporal utility the player receives from the payoff stream resulting from applying solution concept $\psi$ to TU-games $v^{t}$ and $v^{t+1}$. The utility to a player after transfer $\alpha$ is applied is equal to the intertemporal utility the player receives from a payoff stream resulting from applying the same solution concept $\psi$ to TU-games $v^{t}-\alpha 1_{i}$ and $v^{t+1}+\alpha 1_{i}$. We have the following definition.

Definition 3. Under the first transfer system, we associate with each $\Gamma_{1}=(\mathbf{v}, \mathbf{u})$ and $\psi$ the noncooperative game $G_{1}\left(\Gamma_{1}, \psi\right)=\left(\mathscr{S}_{1}, \ldots, \mathscr{S}_{n} ; \tilde{u}_{1}, \ldots, \tilde{u}_{n}\right)$. For every $i \in N$ we define

(i) strategy set $\mathscr{S}_{i}=\left\{\alpha_{i} \in \mathbb{R}^{T} \mid v^{t}(i)+\alpha_{i}^{t} \geq 0 \forall t \in\{1, \ldots, T\}\right.$ and $\left.\sum_{t=1}^{T} \alpha_{i}^{t}=0\right\}$.

(ii) utility function $\tilde{u}_{i}: \mathscr{S}_{N}{ }^{6} \rightarrow \mathbb{R}$, where

$$
\tilde{u}_{i}\left(\alpha_{1}, \ldots, \alpha_{n}\right)=u_{i}\left(\psi_{i}\left(v^{1}+\sum_{j \in N} \alpha_{j}^{1} 1_{j}\right), \ldots, \psi_{i}\left(v^{T}+\sum_{j \in N} \alpha_{j}^{T} 1_{j}\right)\right) .
$$

If payoffs are transferred according to the second transfer system $(k=2)$, a player's transfer does not only change his individual worths, but also the worths of all coalitions that contain him. We assume that an individual transfer ceteris paribus changes all worths of coalitions which contain the individual by an amount equal to the individual transfer. A transfer of size $\alpha$ by player $i$ from game $v^{t}$ to game $v^{t+1}$ results in games $v^{t}-\alpha 2_{i}$ and $v^{t}+\alpha 2_{i}$ were the game $2_{i}$ has worth $2_{i}(S)=1$ if $i \subseteq S$ and $2_{i}(S)=0$ otherwise. The utility to a player before

${ }^{6} \mathscr{S}_{T}=\times_{i \in T} \mathscr{S}_{i}$ for all $T \subseteq N$ 
transfer $\alpha$ is applied is equal to the intertemporal utility the player receives from the payoff stream resulting from applying solution concept $\psi$ to TU-games $v^{t}$ and $v^{t+1}$. The utility to a player after transfer $\alpha$ is applied is equal to the intertemporal utility the player receives from a payoff stream resulting from applying the same solution concept $\psi$ to TU-games $v^{t}-\alpha 2_{i}$ and $v^{t+1}+\alpha 2_{i}$. This implies that within a TU-game, the change of a coalition's worth is equal to the sum of changes of the relevant individual worths.

To ensure that the worths of all coalitions remain nonnegative under the second transfer system, only dynamic TU-games with weakly superadditive TU-games are considered. By imposing weak superadditivity, the worth of a coalition consists of the individual worths of the players contained in the coalition and a nonnegative worth generated by a nonnegative synergy which is a result of players working together. In other words, the worth of a coalition is build up by a transferable part - the individual worths of the relevant players - and a nontransferable part - namely the worth resulting from a nonnegative synergy. This idea supports the second transfer system. We have the following definition.

Definition 4. Under the second transfer system, we associate with each $\Gamma_{2}=$ $(\mathbf{v}, \mathbf{u})$ with $v^{t} \in \mathscr{G}_{+}^{N}$ weakly superadditive for all $t \in\{1, \ldots, T\}$ and $\psi$ the noncooperative game $G_{2}\left(\Gamma_{2}, \psi\right)=\left(\mathscr{S}_{1}, \ldots, \mathscr{S}_{n} ; \tilde{u}_{1}, \ldots, \tilde{u}_{n}\right)$. For every $i \in N$ we define

(i) strategy set $\mathscr{S}_{i}=\left\{\alpha_{i} \in \mathbb{R}^{T} \mid v^{t}(i)+\alpha_{i}^{t} \geq 0 \forall t \in\{1, \ldots, T\}\right.$ and $\left.\sum_{t=1}^{T} \alpha_{i}^{t}=0\right\}$.

(ii) utility function $\tilde{u}_{i}: \mathscr{S}_{N} \rightarrow \mathbb{R}$, where

$$
\tilde{u}_{i}\left(\alpha_{1}, \ldots, \alpha_{n}\right)=u_{i}\left(\psi_{i}\left(v^{1}+\sum_{j \in N} \alpha_{j}^{1} 2_{j}\right), \ldots, \psi_{i}\left(v^{T}+\sum_{j \in N} \alpha_{j}^{T} 2_{j}\right)\right) .
$$

In this chapter we are interested in Nash equilibria of $G_{k}\left(\Gamma_{k}, \psi\right)$ for transfer system $k \in\{1,2\}$. A Nash equilibrium is a strategy profile $\left(\alpha_{1}^{*}, \ldots, \alpha_{n}^{*}\right) \in \mathscr{S}_{N}$ such that for each $i \in N$ it holds that $\tilde{u}_{i}\left(\alpha_{i}, \alpha_{N \backslash i}^{*}\right) \leq \tilde{u}_{i}\left(\alpha_{i}^{*}, \alpha_{N \backslash i}^{*}\right)$ for all $\alpha_{i} \in \mathscr{S}_{i}$. However, a Nash equilibrium does not have to exist as Example 12 later shows.

We first consider weakly and strictly dominant strategies. A strategy $\alpha_{i}^{*} \in \mathscr{S}_{i}$ of player $i$ is weakly dominant if $\tilde{u}_{i}\left(\alpha_{i}^{*}, \alpha_{N \backslash i}\right) \geq \tilde{u}_{i}\left(\alpha_{i}, \alpha_{N \backslash i}\right)$ for all $\alpha_{i} \in \mathscr{S}_{i}$ and all $\alpha_{N \backslash i} \in \mathscr{S}_{N \backslash i}$. If $\tilde{u}_{i}\left(\alpha_{i}^{*}, \alpha_{N \backslash i}\right)>\tilde{u}_{i}\left(\alpha_{i}, \alpha_{N \backslash i}\right)$ for all $\alpha_{i} \in \mathscr{S}_{i}, \alpha_{i} \neq \alpha_{i}^{*}$ and all $\alpha_{N \backslash i} \in \mathscr{S}_{N \backslash i}$, then $\alpha_{i}^{*} \in \mathscr{S}_{i}$ is player $i$ 's strictly dominant strategy. Observe that a combination of weakly dominant strategies is a Nash equilibrium. If these strategies are strictly dominant, then this Nash equilibrium is unique. 
In the next theorem we consider dominant strategies in $G_{1}\left(\Gamma_{1}, \psi\right)$. We show that the strategy to transfer as much payoff as possible to the first time period is a weakly dominant strategy for player $i$.

Theorem 2. Let $i \in N$, let $\psi_{i}(z)=0$ and let $\psi$ be additive.

(i) If $u_{i} \in V$ and if $\psi_{i}$ is increasing in $v(i)$ for all $v \in \mathscr{G}^{N}$, then $\left(\sum_{t=2}^{T} v^{t}(i),-v^{2}(i), \ldots,-v^{T}(i)\right)$ is a weakly dominant strategy for playeri in $G_{1}\left(\Gamma_{1}, \psi\right)$.

(ii) If $u_{i} \in \bar{V}$ and if $\psi_{i}$ is strictly increasing in $v(i)$ for all $v \in \mathscr{G}^{N}$, then $\left(\sum_{t=2}^{T} v^{t}(i),-v^{2}(i), \ldots,-v^{T}(i)\right)$ is player $i$ 's strictly dominant strategy in $G_{1}\left(\Gamma_{1}, \psi\right)$.

Proof. (i) Let $\psi_{i}$ be increasing in $v(i)$ for all $v \in \mathscr{G}^{N}$. To determine player $i$ 's dominant strategies, player $i$ is fixed and the strategies of all other players $N \backslash i$ are considered to be given. Without loss of generality it is assumed that the game $v^{t} \in \mathscr{G}_{+}^{N}$ for every $t$ includes the transfers resulting from all strategies of players $N \backslash i$. By additivity of $\psi$ we can assume without loss of generality that $v^{t}=z$ for all $t$. Because $\psi_{i}$ is increasing in $v(i)$ for all $v \in \mathscr{G}^{N}$ and $\psi_{i}(z)=0$, it follows that $\psi_{i}\left(1_{i}\right) \geq 0$. From Lemma 9 it follows that $u_{i}\left(\psi_{i}\left(v^{1}+\right.\right.$ $\left.\left.\alpha_{i}^{1} 1_{i}\right), \ldots, \psi_{i}\left(v^{T}+\alpha_{i}^{T} 1_{i}\right)\right)=u_{i}\left(\alpha_{i}^{1} \psi_{i}\left(1_{i}\right), \ldots, \alpha_{i}^{T} \psi_{i}\left(1_{i}\right)\right)$ for all $\alpha_{i} \in \mathscr{S}_{i}$.

Take two arbitrary strategies $\beta, \gamma \in \mathscr{S}_{i}$ with $\beta^{t}-\gamma^{t} \leq 0$ for all $t \neq 1$. Then,

$$
\begin{aligned}
u_{i}\left(\beta^{1} \psi_{i}\left(1_{i}\right), \ldots, \beta^{T} \psi_{i}\left(1_{i}\right)\right) & =u_{i}\left(\psi_{i}\left(1_{i}\right)\left(\beta^{1}, \ldots, \beta^{T}\right)\right) \\
& \geq u_{i}\left(\psi_{i}\left(1_{i}\right)\left(\beta^{1}, \ldots, \beta^{T-1}+\left(\beta^{T}-\gamma^{T}\right), \gamma^{T}\right)\right) \\
& \vdots \\
& \geq u_{i}\left(\psi_{i}\left(1_{i}\right)\left(\beta^{1}+\sum_{t=2}^{T}\left(\beta^{t}-\gamma^{t}\right), \ldots, \gamma^{T}\right)\right) \\
& =u_{i}\left(\psi_{i}\left(1_{i}\right)\left(\gamma^{1}, \ldots, \gamma^{T}\right)\right) .
\end{aligned}
$$

The first equality follows from scalar multiplication. The first inequality follows from $u_{i} \in V$ and because $\left(\beta^{T}-\gamma^{T}\right) \psi_{i}\left(1_{i}\right) \leq 0$. The dots represent the second till the penultimate inequality. The second inequality follows from $u_{i} \in V$ and because $\left(\beta^{T-1}-\gamma^{T-1}\right) \psi_{i}\left(1_{i}\right) \leq 0$, etc. The equality at the end follows from $\sum_{t=1}^{T} \beta^{t}=\sum_{t=1}^{T} \gamma^{t}=0$. Because $\beta, \gamma \in \mathscr{S}_{i}$ it holds that $\beta^{t}, \gamma^{t} \geq-v^{t}(i)$ for all $t$. Choose $\left(\beta^{1}, \beta^{2}, \ldots, \beta^{T}\right)=\left(\sum_{t=2}^{T} v^{t}(i),-v^{2}(i), \ldots,-v^{T}(i)\right)$, then (4.2) holds for all 
$\gamma \in \mathscr{S}_{i}$ because $\beta^{t}-\gamma^{t} \leq 0$ for all $t \neq 1$. As a result $\left(\sum_{t=2}^{T} v^{t}(i),-v^{2}(i), \ldots,-v^{T}(i)\right)$ is a weakly dominant strategy for player $i$ in $G_{1}\left(\Gamma_{1}, \psi\right)$.

(ii) Let $\psi_{i}$ be strictly increasing in $v(i)$ for all $v \in \mathscr{G}^{N}$, then $\psi_{i}\left(1_{i}\right)>0$ since $\psi_{i}(z)=0$. The remainder of the proof of part (ii) follows the proof of part (i), however we take two arbitrary strategies $\beta, \gamma \in \mathscr{S}_{i}$ with $\beta^{t}-\gamma^{t} \leq 0$ for all $t \neq 1$ and $\beta^{t}-\gamma^{t}<0$ for at least one $t \neq 1$. Because $u_{i} \in \bar{V}$ it then follows that at least one of the inequality signs $(\geq)$ in (4.2) should be replaced by a strict inequality $\operatorname{sign}(>)$. As a result $\left(\sum_{t=2}^{T} v^{t}(i),-v^{2}(i), \ldots,-v^{T}(i)\right)$ is player $i$ 's strictly dominant strategy in $G_{1}\left(\Gamma_{1}, \psi\right)$.

The next theorem shows when the condition $\psi_{i}$ is increasing in the individual worth of player $i$ is a necessary condition.

Theorem 3. Let $\psi$ be additive. Let $i \in N, u_{i} \in V$ and let $\psi_{i}$ be continuous in $v(i)$ for all $v \in \mathcal{G}^{N}$. Let $\left(\sum_{t=2}^{T} v^{t}(i),-v^{2}(i), \ldots,-v^{T}(i)\right)$ be a weakly dominant strategy of player $i$ for all $\Gamma_{1}=(\mathbf{v}, \mathbf{u})$ in $G_{1}\left(\Gamma_{1}, \psi\right)$. Then $\psi_{i}$ is increasing in $v(i)$ for all $v \in \mathscr{G}_{+}^{N}$.

Proof. Fix $i \in N$. Assume, contrary to what we want to show, that $\psi_{i}$ is not increasing in $w(i)$ for all $w \in \mathscr{G}_{+}^{N}$. By definition this is similar to assuming that $\psi_{i}$ is strictly decreasing in $w(i)$ for all $w \in \mathscr{G}_{+}^{N}$. Hence, suppose there exist $v, v^{\prime} \in$ $\mathscr{G}_{+}^{N}$ with $v(i)>v^{\prime}(i), v(S)=v^{\prime}(S)$ for all $S \neq\{i\}$ and $\psi_{i}(\nu)<\psi_{i}\left(\nu^{\prime}\right)$. Then,

$$
0<\psi_{i}\left(v^{\prime}\right)-\psi_{i}(v)=\psi_{i}\left(v^{\prime}-v\right)=\psi_{i}\left(\left(v^{\prime}(i)-v(i)\right) 1_{i}\right)=\left(v^{\prime}(i)-v(i)\right) \psi_{i}\left(1_{i}\right) .
$$

The first equality follows by additivity of $\psi$. The second equality follows by definition. The last equality follows from Lemma 8(i) and (ii). As a result $\psi_{i}\left(1_{i}\right)<0$, by $v(i)>v^{\prime}(i)$. Thus, for every $w, w^{\prime} \in \mathscr{G}_{+}^{N}$ with $w(i)>w^{\prime}(i)$ and $w(S)=w^{\prime}(S)=$ $v(S)\left(=v^{\prime}(S)\right)$ for all $S \neq\{i\}$, we have

$$
\psi_{i}\left(w^{\prime}\right)-\psi_{i}(w)=\psi_{i}\left(w^{\prime}-w\right)=\psi_{i}\left(\left(w^{\prime}(i)-w(i)\right) 1_{i}\right)=\left(w^{\prime}(i)-w(i)\right) \psi_{i}\left(1_{i}\right)>0 .
$$

The first equality follows from additivity of $\psi$. The second equality follows by definition. The third equality results from Lemma 8(i) and (ii). Finally, the inequality follows from the fact that $\psi_{i}\left(1_{i}\right)<0$ and $w(i)>w^{\prime}(i)$.

Take $w, w^{\prime}$ with $w(i)=1, w^{\prime}(i)=0$ and $w(S)=w^{\prime}(S)=v(S)\left(=v^{\prime}(S)\right)$ for every $S \neq\{i\}$. Consider the sequence of TU-games $\left(w, w^{\prime}, z, \ldots, z\right) \in\left(\mathscr{G}_{+}^{N}\right)^{T}$. In this case $\left(w^{\prime}(i),-w^{\prime}(i), 0, \ldots, 0\right)=(0,0, \ldots, 0) \in \mathscr{S}_{i}$ is a weakly dominant strategy 
of player $i$. Then

$$
\begin{aligned}
& u_{i}\left(\psi_{i}(w), \psi_{i}\left(w^{\prime}\right), \psi_{i}(z), \ldots, \psi_{i}(z)\right) \\
& \quad \geq u_{i}\left(\psi_{i}\left(w+\left(w^{\prime}(i)-w(i)\right) 1_{i}\right), \psi_{i}\left(w^{\prime}-\left(w^{\prime}(i)-w(i)\right) 1_{i}\right), \ldots, \psi_{i}(z)\right) \\
& \quad=u_{i}\left(\psi_{i}\left(w^{\prime}\right), \psi_{i}(w), \psi_{i}(z), \ldots, \psi_{i}(z)\right),
\end{aligned}
$$

which is true only if $\left(w^{\prime}(i)-w(i)\right) \psi_{i}\left(1_{i}\right) \leq 0$. However, this contradicts our assumption that $\psi_{i}$ is decreasing in $w(i)$ for all $w \in \mathscr{G}_{+}^{N}$, since in that case $w^{\prime}(i)-w(i)=-1$ and $\psi_{i}\left(1_{i}\right)<0$. However, since we assumed the opposite $0 \geq\left(w^{\prime}(i)-w(i)\right) \psi_{i}\left(1_{i}\right)=\psi_{i}\left(\left(w^{\prime}(i)-w(i)\right) 1_{i}\right)=\psi_{i}\left(w^{\prime}-w\right)=\psi_{i}\left(w^{\prime}\right)-\psi_{i}(w)$. The first equality follows from Lemma 8(i) and (ii). The second equality follows by definition. The last equality follows by additivity of $\psi_{i}$. Thus, $\psi_{i}$ is increasing in $w(i)$ for all $w \in \mathscr{G}_{+}^{N}$.

In the following theorem we consider dominant strategies in $G_{2}\left(\Gamma_{2}, \psi\right)$

Theorem 4. Let $i \in N$, let $\psi_{i}(z)=0$ and let $\psi$ be restrictively additive.

(i) If $u_{i} \in V$, then $\left(\sum_{t=2}^{T} v^{t}(i),-v^{2}(i), \ldots,-v^{T}(i)\right)$ is a weakly dominant strategy for player $i$ in $G_{2}\left(\Gamma_{2}, \psi\right)$.

(ii) If $u_{i} \in \bar{V}$, then $\left(\sum_{t=2}^{T} v^{t}(i),-v^{2}(i), \ldots,-v^{T}(i)\right)$ is player $i$ 's strictly dominant strategy in $G_{2}\left(\Gamma_{2}, \psi\right)$.

Proof. (i) Fix player $i$. For all $\alpha \in \mathscr{S}_{N}$ it holds that $\psi_{i}\left(v^{t}+\sum_{j \in N} \alpha_{j}^{t} 2_{j}\right)=\psi_{i}\left(v^{t}\right)+$ $\alpha_{i}^{t}$ for all $j \in N$ and all $t$, because $\psi$ is restrictively additive and $\psi_{i}(z)=0$. Take two arbitrary strategies $\beta, \gamma \in \mathscr{S}_{i}$ with $\beta^{t}-\gamma^{t} \leq 0$ for $t \neq 1$. Then it follows that

$$
\begin{aligned}
& u_{i}\left(\psi_{i}\left(v^{1}\right)+\beta^{1}, \ldots, \psi_{i}\left(v^{T}\right)+\beta^{T}\right) \\
& \quad \geq u_{i}\left(\psi_{i}\left(v^{1}\right)+\beta^{1}, \ldots, \psi_{i}\left(v^{T-1}\right)+\beta^{T-1}+\beta^{T}-\gamma^{T}, \psi_{i}\left(v^{T}\right)+\gamma^{T}\right) \\
& \quad \vdots \\
& \quad \geq u_{i}\left(\psi_{i}\left(v^{1}\right)+\beta^{1}+\sum_{t=2}^{T}\left(\beta^{t}-\gamma^{t}\right), \psi_{i}\left(v^{2}\right)+\gamma^{2}, \ldots, \psi_{i}\left(v^{T}\right)+\gamma^{T}\right) \\
& \quad=u_{i}\left(\psi_{i}\left(v^{1}\right)+\gamma^{1}, \ldots, \psi_{i}\left(v^{T}\right)+\gamma^{T}\right) .
\end{aligned}
$$

The first inequality follows from $u_{i} \in V$ and since $\beta^{T}-\gamma^{T} \leq 0$. The vertical dots represent the second till the penultimate inequality. The second inequality follows from $u_{i} \in V$ and because $\beta^{T-1}-\gamma^{T-1} \leq 0$, etc. The last inequality follows from $u_{i} \in V$ and $\beta^{2}-\gamma^{2} \leq 0$. Finally, the equality follows because 
$\sum_{t=1}^{T} \beta^{t}=\sum_{t=1}^{T} \gamma^{t}=0$. Since $\beta, \gamma \in \mathscr{S}_{i}$ and $v^{t} \in \mathscr{G}_{+}^{N}$ is weakly superadditive for all $t$, it follows that $\beta^{t}, \gamma^{t} \geq-v^{t}(i)$ for all $i \in N$ and all $t$. Hence, if $\left(\beta^{1}, \beta^{2}, \ldots, \beta^{T}\right)$ is chosen equal to $\left(\sum_{t=2}^{T} v^{t}(i),-v^{2}(i), \ldots,-v^{T}(i)\right)$, then for all $\gamma \in \mathscr{S}_{i}$ it follows that (4.3) holds, since $\beta^{t}-\gamma^{t} \leq 0$ for $t \neq 1$. Thus $\left(\sum_{t=2}^{T} v^{t}(i),-v^{2}(i), \ldots,-v^{T}(i)\right)$ is a weakly dominant strategy for player $i$ in $G_{2}\left(\Gamma_{2}, \psi\right)$.

(ii) The proof of part (ii) follows the line of proof of part (i), however take $\beta^{t}-\gamma^{t} \leq 0$ for all $t \neq 1$ and $\beta^{t}-\gamma^{t}<0$ for at least one $t \neq 1$. Because $u_{i} \in \bar{V}$ it then follows that at least one of the inequality signs $(\geq)$ in (4.3) should be replaced by a strict inequality sign $(>)$. In a similar way as in part (i) we then find that $\left(\sum_{t=2}^{T} v^{t}(i),-v^{2}(i), \ldots,-v^{T}(i)\right)$ is player $i$ 's strictly dominant strategy in $G_{2}\left(\Gamma_{2}, \psi\right)$.

Obviously, if the conditions in Theorem 2 apply to all players $i \in N$, then there is at least one Nash equilibrium in dominant strategies. The same is true for the case that all players satisfy the conditions mentioned in Theorem 4 . In the resulting equilibrium all players transfer as much payoff as possible to the first time period. If for some (or all) players receiving payoff in the first time period is valued less than receiving the payoff in another time period, then a Nash equilibrium in dominant strategies is obtained in which every player transfers as much payoff as possible to the time period in which he values receiving payoff most.

In Theorem 4 a restrictively additive solution is considered. The nucleolus and prenucleolus are restrictively additive. Specifically, they are covariant (Peleg and Sudhölter [30]). The nucleolus is a one-point payoff distribution which is defined for games in $\mathscr{G}^{N}$ with a non-empty imputation set. The imputation set contains all payoffs which are efficient and individually rational for all players. If the core of a game is nonempty then the nucleolus is in the core. In case the core is nonempty, the nucleolus and prenucleolus coincide. The prenucleolus is also defined if the imputation set is empty; it always exists.

Both solutions are defined as the solution of a minimization problem, where excesses of all nonempty coalitions of a game are lexicographically minimized. The excess of coalition $S$ in game $v \in \mathscr{G}^{N}$ given payoff vector $(x(1), \ldots, x(n)) \in$ $\mathbb{R}^{N}$ is defined as $v(S)-\sum_{i \in S} x(i)$ for all $v \in \mathscr{G}^{N}$. The nucleolus as well as the prenucleolus satisfy the efficiency property, i.e. $\sum_{i \in N} x(i)=v(N)$. Additionally, the nucleolus satisfies the property of individual rationality for all players 
which means that $x(i) \geq v(i)$ for all $i \in N .^{7}$

If the (pre)nucleolus is applied to the dynamic TU-game $\Gamma_{2}=(\mathbf{v}, \mathbf{u})$ where $u_{i} \in V$ for player $i \in N$, then transferring as much payoff as possible to the first time period is a weakly dominant strategy of player $i$ in case payoffs are transferred according to the second transfer system. However, as the following example illustrates, this implication does not have to hold in case the first transfer system is used. In particular it is shown that transferring as much as possible to $t=1$ is not at all beneficial for player 1 .

Example 9. Consider the dynamic TU-game $\Gamma_{1}=\left(v^{1}, v^{2} ; u_{1}, u_{2}, u_{3}\right)$, the nucleolus $\psi$ and the first transfer system. The game $v^{1}$ is defined as follows: $v^{1}(1)=0$; $v^{1}(2)=2 ; v^{1}(3)=3 ; v^{1}(12)=4 ; v^{1}(13)=v^{1}(23)=5$ and $v^{1}(N)=7$, and the game $v^{2}$ has $v^{2}(1)=1 ; v^{2}(2)=v^{2}(3)=0 ; v^{2}(12)=v^{2}(13)=v^{2}(23)=2$ and $v^{2}(N)=4$. The functions $u_{1}, u_{2}$ and $u_{3}$ are respectively player 1, player 2 and player 3's intertemporal utility functions. Furthermore $\psi\left(v^{1}\right)=(2,2,3)$ and $\psi\left(v^{2}\right)=\left(\frac{3}{2}, \frac{5}{4}, \frac{5}{4}\right)$. Suppose that player 1 transfers $\alpha=(1,-1)$, which means that he transfers the maximum amount of payoff possible to his first time period. Then the games $v^{1}+\alpha^{1} 1_{1}$ and $v^{2}+\alpha^{2} 1_{1}$ are obtained and accordingly $\psi\left(v^{1}+1 \cdot 1_{1}\right)=(2,2,3)$ and $\psi\left(v^{2}-1 \cdot 1_{1}\right)=\left(\frac{4}{3}, \frac{4}{3}, \frac{4}{3}\right)$. Thus, player 1 receives payoff 2 in the first period and payoff $\frac{3}{2}$ in the second, before any transfer is made. After he makes the transfer $\alpha=(1,-1)$, he receives a payoff equal to 2 in the first period and only a payoff of $\frac{4}{3}$ in the second period. Under any reasonable utility function, transferring $(1,-1)$ is clearly not beneficial for player 1 .

Since the cores of the TU-games $v^{1}$ and $v^{2}$ in example 9 are nonempty before as well as after player 1 has made a transfer, the prenucleolus and nucleolus coincide. Therefore this example also applies to the prenucleolus. In example 10, the first transfer system is applied to the dynamic TU-game $\Gamma_{1}=(\mathbf{v}, \mathbf{u})$ over which the nucleolus is calculated to show that an extreme strategy profile (i.e a strategy profile where all payoff is transferred to either the first time period or the last time period) does not have to be a Nash equilibrium. As in example 9 the cores for the TU-games $v^{1}$ and $v^{2}$ before as well as after payoffs are transferred are nonempty and therefore the nucleolus and prenucleolus coincide.

Example 10. The dynamic TU-game $\Gamma_{1}=\left(v^{1}, v^{2} ; u_{1}, u_{2}, u_{3}\right)$ is considered, the nucleolus $\psi$ and the first transfer system. The function $u_{1}$ is a constant discounted intertemporal utility function with discount factor $0 \leq \delta \leq 1$. The game

\footnotetext{
${ }^{7}$ See Peleg and Sudhölter [30] for more information about the nucleolus and prenucleolus.
} 
$v^{1}$ is described by $v^{1}(1)=v^{1}(2)=v^{1}(3)=0$ and $v^{1}(12)=v^{1}(13)=v^{1}(23)=\frac{2}{3}$ and $v^{1}(N)=3$. The game $v^{2}$ is defined as $v^{2}(1)=2$ and $v^{2}(S)=v^{1}(S)$ for all $S \neq\{1\}$. Clearly only player 1 can make transfers between the two time periods, which are denoted by $\alpha \in \mathbb{R}^{2}$ where $\alpha^{1}+\alpha^{2}=0$ and $\alpha^{2} \geq-2$. Utility function $u_{1}\left(\psi\left(v^{1}+\alpha^{1} 1_{1}\right), \psi\left(v^{2}+\alpha^{2} 1_{1}\right)\right)=\psi\left(v^{1}+\alpha^{1} 1_{1}\right)+\delta \psi\left(v^{2}+\alpha^{2} 1_{1}\right)$ is contained in the class $V$ for $\delta \leq 1$. If $\frac{3}{4}<\delta \leq 1$, then none of the two extreme transfers $\alpha^{1}=0$ and $\alpha^{1}=2$ give player 1 the highest possible utility, see Table 4.1.

Table 4.1: Computation of nucleoli for different transfers.

\begin{tabular}{rrr}
\hline$\alpha^{1}$ & $\psi\left(v^{1}+\alpha^{1} 1_{1}\right)$ & $\psi\left(v^{2}+\alpha^{2} 1_{1}\right)$ \\
\hline 0 & $(1,1,1)$ & $\left(2 \frac{1}{6}, \frac{5}{12}, \frac{5}{12}\right)$ \\
1 & $\left(1 \frac{2}{3}, \frac{2}{3}, \frac{2}{3}\right)$ & $\left(1 \frac{2}{3}, \frac{2}{3}, \frac{2}{3}\right)$ \\
2 & $\left(2 \frac{1}{6}, \frac{5}{12}, \frac{5}{12}\right)$ & $(1,1,1)$ \\
\hline
\end{tabular}

Debreu [15] proves existence of a Nash equilibrium in a noncooperative game with finitely many players, whose strategy sets are convex and compact subsets of a Euclidian space and whose utility functions are continuous and quasi-concave. To apply Debreu's theorem to $G_{k}\left(\Gamma_{k}, \psi\right)$ for $k \in\{1,2\}$ extra conditions are necessary in order to prove existence.

The value $\psi$ is transfer-1-concave, if for all $i \in N$ and all $v, w, w^{\prime} \in \mathscr{G}^{N}$

$$
\psi_{i}\left(\lambda(\nu+w)+(1-\lambda)\left(\nu+w^{\prime}\right)\right) \geq \lambda \psi_{i}(\nu+w)+(1-\lambda) \psi_{i}\left(\nu+w^{\prime}\right)
$$

where $w, w^{\prime}$ are generated by $(w(1), \ldots, w(n))$ and $\left(w^{\prime}(1), \ldots, w^{\prime}(n)\right)$ contained in $\mathbb{R}^{N}$, namely $w=\sum_{j \in N} w(j) \mathbf{1}_{j}$ and $w^{\prime}=\sum_{j \in N} w^{\prime}(j) \mathbf{1}_{j}$.

The value $\psi$ is transfer-2-concave, if for all $i \in N$ and all $v, w, w^{\prime} \in \mathscr{G}^{N}$

$$
\psi_{i}\left(\lambda(\nu+w)+(1-\lambda)\left(\nu+w^{\prime}\right)\right) \geq \lambda \psi_{i}(\nu+w)+(1-\lambda) \psi_{i}\left(\nu+w^{\prime}\right)
$$

where $w, w^{\prime}$ are additive, generated by $(w(1), \ldots, w(n))$ and $\left(w^{\prime}(1), \ldots, w^{\prime}(n)\right)$ contained in $R^{N}$, which means that $w=\sum_{j \in N} w(j) 2_{j}$ and $w^{\prime}=\sum_{j \in N} w^{\prime}(j) 2_{j}$.

Lemma 10. Let $k \in\{1,2\}$, let $i \in N$, let $u_{i}$ be an increasing and quasi-concave function and let $\psi$ be transfer- $k$-concave. Then $\tilde{u}_{i}$ is quasi-concave. 
Proof. (i) Let $k=1$. This means that for all $\beta, \gamma \in \mathscr{S}_{N}$ and $\lambda \in(0,1)$ it holds that $\psi_{i}\left(\lambda\left(v^{t}+\sum_{j \in N} \beta_{j}^{t} 1_{j}\right)+(1-\lambda)\left(v^{t}+\sum_{j \in N} \gamma_{j}^{t} 1_{j}\right)\right) \geq \lambda \psi_{i}\left(v^{t}+\sum_{j \in N} \beta_{j}^{t} 1_{j}\right)+(1-$ $\lambda) \psi_{i}\left(\nu^{t}+\sum_{j \in N} \gamma_{j}^{t} 1_{j}\right)$ for all $t$ and all $i \in N$.

Then,

$$
\begin{aligned}
\tilde{u}_{i}(\lambda \beta & +(1-\lambda) \gamma)=u_{i}\left(\psi_{i}\left(v^{t}+\sum_{j \in N}\left[\lambda \beta_{j}^{t}+(1-\lambda) \gamma_{j}^{t}\right] 1_{j}\right) \forall t \in\{1, \ldots, T\}\right)= \\
& =u_{i}\left(\psi_{i}\left(\lambda\left[v^{t}+\sum_{j \in N} \beta_{j}^{t} 1_{j}\right]+(1-\lambda)\left[v^{t}+\sum_{j \in N} \gamma_{j}^{t} 1_{j}\right]\right) \forall t \in\{1, \ldots, T\}\right) \\
& \geq u_{i}\left(\left[\lambda \psi_{i}\left(v^{t}+\sum_{j \in N} \beta_{j}^{t} 1_{j}\right)+(1-\lambda) \psi_{i}\left(v^{t}+\sum_{j \in N} \gamma_{j}^{t} 1_{j}\right)\right] \forall t \in\{1, \ldots, T\}\right) \\
& \geq \min \left\{u_{i}\left(\psi_{i}\left(v^{t}+\sum_{j \in N} \beta_{j}^{t} 1_{j}\right) \forall t \in\{1, \ldots, T\}\right), u_{i}\left(\psi_{i}\left(v^{t}+\sum_{j \in N} \gamma_{j}^{t} 1_{j}\right) \forall t \in\{1, \ldots, T\}\right)\right\} \\
& =\min \left\{\tilde{u}_{i}(\beta), \tilde{u}_{i}(\gamma)\right\} .
\end{aligned}
$$

The first equality follows from the definition of $\tilde{u}_{i}$. The first inequality follows from transfer-1-concavity of $\psi$ and because $u_{i}$ is an increasing function. The second inequality follows from quasi-concavity of $u_{i}$. The last equality follows from the definition of $\tilde{u}_{i}$.

(ii) Let $k=2$. Then $1_{j}$ in part (i) is replaced by $2_{j}$. In an analogous way as part (i) it follows that $\tilde{u}_{i}$ is quasi-concave.

Lemma 10 is used in Theorem 5 below to prove existence of a Nash equilibrium.

Theorem 5. Let $k \in\{1,2\}$, let $u_{i}$ be continuous, increasing and quasi-concave for all $i \in N$, let $\psi$ be continuous and transfer- $k$-concave. Then a Nash equilibrium exists in $G_{k}\left(\Gamma_{k}, \psi\right)$.

Proof. From continuity of $\psi$ and continuity of $u_{i}$ it follows that $\tilde{u}_{i}$ is a continuous function for all $i \in N$. Since the set $\mathscr{S}_{i}$ is compact for all $i \in N$ it follows that $\mathscr{S}_{N}$ is compact. A convex combination of strategies in $\mathscr{S}_{N}$ is contained in $\mathscr{S}_{N}$ and therefore $\mathscr{S}_{N}$ is convex. From Lemma 10 it follows that $\tilde{u}_{i}$ is quasi-concave for all $i \in N$. We can apply Debreu's existence Theorem [15] from which it follows that a Nash Equilibrium exists in $G_{k}\left(\Gamma_{k}, \psi\right)$ for $k \in\{1,2\}$.

One of the conditions in Lemma 10 and Theorem 5 is transfer- $k$-concavity of the solution $\psi$. For $k \in\{1,2\}, \psi$ is transfer- $k$-concave in case the solution $\psi$ is additive, $\psi_{i}$ is increasing in $v(i)$ for all $i \in N$ and all $v \in \mathscr{G}^{N}$ and $\psi_{i}(z)=0$ for all $i \in N$. Additionally, $\psi$ is transfer-2-concave if $\psi$ is restrictively additive and 
$\psi_{i}(z)=0$ for all $i \in N$. Example 11 illustrates that the nucleolus, a restrictively additive solution, is not transfer-1-concave.

Example 11. Consider the game $v$ where $v(1)=0, v(2)=1, v(3)=0$ and $v(12)=$ $1, v(13)=2, v(23)=1$ and $v(N)=6$. The first transfer system is used and only transfers of player 1 are considered. The nucleolus $\psi\left(v+\alpha 1_{1}\right)$ can be computed for different values $\alpha$ and $\psi\left(v+0 \cdot 1_{1}\right)=\psi(v)=\left(\frac{7}{4}, \frac{5}{2}, \frac{7}{4}\right), \psi\left(v+\frac{1}{2} \cdot 1_{1}\right)=\left(2, \frac{5}{2}, \frac{3}{2}\right)$ and $\psi\left(v+1 \cdot 1_{1}\right)=\left(\frac{7}{3}, \frac{7}{3}, \frac{4}{3}\right)$ are obtained.

Take $\lambda=\frac{1}{2}$ and strategies $x=0$ and $y=1$ for player 1 , then

$$
\psi_{1}\left(\lambda\left(\nu+x 1_{1}\right)+(1-\lambda)\left(v+y 1_{1}\right)\right)=\psi_{1}\left(\nu+\frac{1}{2} \cdot 1_{1}\right)=2 .
$$

However,

$$
\lambda \psi_{1}\left(\nu+x 1_{1}\right)+(1-\lambda) \psi_{1}\left(v+y 1_{1}\right)=\frac{1}{2}\left(\frac{7}{4}+\frac{7}{3}\right)=\frac{49}{24} .
$$

Since $2 \ngtr \frac{49}{24}$, it follows that the nucleolus is not transfer-1-concave.

In Example 11 the cores of the games $v+\alpha 1_{i}$ for $\alpha \in\left\{0, \frac{1}{2}, 1\right\}$ are nonempty and therefore the prenucleolus and nucleolus coincide. This means that both solutions are not transfer-1-concave and thus Theorem 5 cannot be used. In Example 12 we consider the dynamic game $\Gamma_{1}=(\mathbf{v}, \mathbf{u})$ where the intertemporal utility function $u_{i}$ is continuous and contained in class $V$ for all $i \in N$. Payoffs are transferred according to the first transfer system and the solution which is used is restrictively additive. In the example a Nash equilibrium does not exist. This is consistent with the fact that the nucleolus is not transfer-1-concave which thereby causes at least one of the utility functions, $\tilde{u}_{i}$ for $i \in N$, of the associated noncooperative game to be not quasi-concave.

Example 12. Consider the dynamic TU-game $\Gamma_{1}=\left(v^{1}, v^{2} ; u_{1}, u_{2}, u_{3}\right)$, the nucleolus $\psi$ and transfer system $k=1$. The game $v^{1}$ is described as $v^{1}(1)=v^{1}(2)=$ $v^{1}(3)=0, v^{1}(12)=v^{1}(23)=1, v^{1}(13)=2$ and $v^{1}(N)=6$ and $v^{2}$ has worths $v^{2}(1)=v^{2}(2)=1, v^{2}(S)=v^{1}(S)$ for all $S \neq\{1\},\{2\}$. Clearly only players 1 and 2 can transfer payoffs between periods 1 and 2, player 3 is just a strategic dummy player. The vectors $\alpha=\left(\alpha^{1}, \alpha^{2}\right) \in \mathscr{S}_{1}$ and $\beta=\left(\beta^{1}, \beta^{2}\right) \in \mathscr{S}_{2}$ contain all possible strategies resulting from transferring payoffs of respectively player 1 and player 2. Moreover, $\alpha^{1}+\alpha^{2}=\beta^{1}+\beta^{2}=1$ and $\alpha^{1}, \beta^{1} \in[0,1]$.

The intertemporal utility functions $u_{1}$ and $u_{2}$ are constant discounted utility functions with a discount factor equal to one and contained in $V$. By definition it holds that $\tilde{u}_{i}(\alpha, \beta)$ in of $G_{1}\left(\Gamma_{1}, \psi\right)$ is equal to $u_{i}\left(\psi\left(v^{1}+\alpha^{1} 1_{1}+\beta^{1} 1_{2}\right), \psi\left(v^{2}+\right.\right.$ $\left.\left.\alpha^{2} 1_{1}+\beta^{2} 1_{2}\right)\right)$ for $i \in\{1,2\}$. 
Table 4.2 shows for every transfer $\beta$ of player 2 , player 1's utility $\tilde{u}_{1}(\alpha, \beta)$. Table 4.3 shows for every possible transfer $\alpha$ of player 1, player 2's utility.

Table 4.2: Player 1's utility function $\tilde{u}_{1}(\alpha, \beta)$.

\begin{tabular}{ll}
\hline value of $\beta^{1}$ & $\tilde{u}_{1}(\alpha, \beta)$ \\
\hline$\beta^{1}=1$ if $\alpha^{1} \in\left[0, \frac{1}{2}\right]$ & $4 \frac{1}{3}-\frac{1}{6} \alpha^{1}+\frac{1}{12} \beta^{1}$ \\
$\beta^{1}=1$ if $\alpha^{1} \in\left[\frac{1}{2}, 1\right]$ & $4 \frac{1}{3}$ \\
$\beta^{1} \in(0,1)$ if $\alpha^{1} \in\left[0, \frac{1}{2} \beta^{1}\right]$ & $4 \frac{1}{3}-\frac{1}{6} \alpha^{1}+\frac{1}{12} \beta^{1}$ \\
$\beta^{1} \in(0,1)$ if $\alpha^{1} \in\left[\frac{1}{2} \beta^{1}, \frac{1}{2} \beta^{1}+\frac{1}{2}\right]$ & $4 \frac{1}{3}$ \\
$\beta^{1} \in(0,1)$ if $\alpha^{1} \in\left[\frac{1}{2} \beta^{1}+\frac{1}{2}, 1\right]$ & $4 \frac{1}{4}+\frac{1}{6} \alpha^{1}-\frac{1}{12} \beta^{1}$ \\
$\beta^{1}=0$ if $\alpha^{1} \in\left[0, \frac{1}{2}\right]$ & $4 \frac{1}{3}$ \\
$\beta^{1}=0$ if $\alpha^{1} \in\left[\frac{1}{2}, 1\right]$ & $4 \frac{1}{4}+\frac{1}{6} \alpha^{1}-\frac{1}{12} \beta^{1}$ \\
\hline
\end{tabular}

Table 4.3: Player 2's utility function $\tilde{u}_{2}(\alpha, \beta)$.

\begin{tabular}{ll}
\hline value of $\alpha^{1}$ & $\tilde{u}_{2}(\alpha, \beta)$ \\
\hline$\alpha^{1} \in\left[0, \frac{1}{2}\right]$ if $\beta^{1} \in\left[0,2 \alpha^{1}\right]$ & $4 \frac{1}{3}$ \\
$\alpha^{1} \in\left[0, \frac{1}{2}\right]$ if $\beta^{1} \in\left[2 \alpha^{1}, 1\right]$ & $4 \frac{1}{3}+\frac{1}{3} \alpha^{1}-\frac{1}{6} \beta^{1}$ \\
$\alpha^{1} \in\left[\frac{1}{2}, 1\right]$ if $\beta^{1} \in\left[0,2 \alpha^{1}-1\right]$ & $4 \frac{1}{2}-\frac{1}{3} \alpha^{1}+\frac{1}{6} \beta^{1}$ \\
$\alpha^{1} \in\left[\frac{1}{2}, 1\right]$ if $\beta^{1} \in\left[2 \alpha^{1}-1,1\right]$ & $4 \frac{1}{3}$ \\
\hline
\end{tabular}

The according reaction functions of player 1 and 2 are given in Table 4.4 and displayed in Figure 4.1. As can be seen, there is no overlap between best response sets of players 1 and 2. Hence, no Nash equilibrium exists.

Table 4.4: Player 1's and 2's reaction functions.

\begin{tabular}{|c|c|c|c|}
\hline \multicolumn{2}{|c|}{ Reaction function player 1} & \multicolumn{2}{|c|}{ Reaction function player 2} \\
\hline value of $\beta^{1}$ & best response $\alpha^{1 *}$ & value of $\alpha^{1}$ & best response $\beta^{1}$ * \\
\hline $\begin{array}{l}\beta^{1} \in\left[0, \frac{1}{2}\right] \\
\beta^{1} \in\left[\frac{1}{2}, 1\right]\end{array}$ & $\begin{array}{l}\alpha^{1 *}=1 \\
\alpha^{1 *}=0\end{array}$ & $\begin{array}{l}\alpha^{1} \in\left[0, \frac{1}{2}\right] \\
\alpha^{1} \in\left[\frac{1}{2}, 1\right]\end{array}$ & $\begin{array}{l}\beta^{1 *} \in\left[0,2 \alpha^{1}\right] \\
\beta^{1 *} \in\left[2 \alpha^{1}-1,1\right]\end{array}$ \\
\hline
\end{tabular}




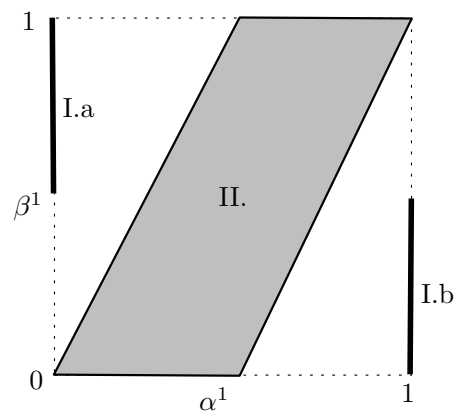

Figure 4.1: Best response sets of players 1 and 2. Player 1's best response set consists of parts I.a and I.b, player 2's best response set consists of part II.

\subsection{Pareto Optimality}

The notion of Nash equilibrium has a number of limitations. A drawback of Nash equilibrium is that it is not immune to coalitional deviations. As a result Pareto optimality of a Nash equilibrium is not guaranteed. In this section Nash equilibria in weakly dominant strategies are considered where players transfer as much payoff as possible to time period 1. In the next theorem we show, given particular conditions considered before, which equilibria are weakly and strongly Pareto optimal. A strategy profile $\alpha^{*} \in \mathscr{S}_{N}$ is weakly Pareto optimal if there exists no strategy profile $\alpha \in \mathscr{S}_{N}$ such that $\tilde{u}_{i}(\alpha)>\tilde{u}_{i}\left(\alpha^{*}\right)$ for all $i \in N$. A strategy profile $\alpha^{*} \in \mathscr{S}_{N}$ is strongly Pareto optimal if there exists no strategy profile $\alpha \in \mathscr{S}_{N}$ such that $\tilde{u}_{i}(\alpha) \geq \tilde{u}_{i}\left(\alpha^{*}\right)$ for all $i \in N$ with at least one strict inequality.

Theorem 6. Let $\psi$ be restrictively additive and $\psi_{i}(z)=0$ for all $i \in N$.

(i) If $u_{i} \in V$ for all $i \in N$, then strategy profile $\left(\sum_{t=2}^{T} v^{t}(i),-v^{2}(i), \ldots,-v^{T}(i)\right)_{i \in N}$ is weakly Pareto optimal in $G_{2}\left(\Gamma_{2}, \psi\right)$.

(ii) If $u_{i} \in \bar{V}$ for all $i \in N$, then strategy profile $\left(\sum_{t=2}^{T} v^{t}(i),-v^{2}(i), \ldots,-v^{T}(i)\right)_{i \in N}$ is strongly Pareto optimal in $G_{2}\left(\Gamma_{2}, \psi\right)$.

Proof. (i) Let $u_{i} \in V$ for all $i \in N$. Since $\psi$ is restrictively additive and $\psi_{i}(z)=$ 0 and $u_{i} \in V$ for all $i \in N$, it follows from Theorem 4(i) that strategy profile $\left(\sum_{t=2}^{T} v^{t}(i),-v^{2}(i), \ldots,-v^{T}(i)\right)_{i \in N}$ is a Nash equilibrium in $G_{2}\left(\Gamma_{2}, \psi\right)$. Moreover, because $u_{i} \in V$ for all $i \in N$, this Nash equilibrium is weakly Pareto optimal. 
This follows since strategy $\left(\sum_{t=2}^{T} v^{t}(i),-v^{2}(i), \ldots,-v^{T}(i)\right)$ generates for every player $i \in N$ his maximum utility, which does not depend on other players' strategies.

(ii) Let $u_{i} \in \bar{V}$ for all $i \in N$. By Theorem 4(ii) it follows that strategy profile $\left(\sum_{t=2}^{T} v^{t}(i),-v^{2}(i), \ldots,-v^{T}(i)\right)_{i \in N}$ is a unique Nash equilibrium in $G_{2}\left(\Gamma_{2}, \psi\right)$. Because $u_{i} \in \bar{V}$ for all $i \in N$, this Nash equilibrium is strongly Pareto optimal. This follows since strategy $\left(\sum_{t=2}^{T} v^{t}(i),-v^{2}(i), \ldots,-v^{T}(i)\right)$ generates for every player $i \in N$ his strictly highest level of utility independent of other players strategies.

The nucleolus as well as the Shapley value are restrictively additive and satisfy the conditions of Theorem 6 . In case we consider noncooperative game $G_{1}\left(\Gamma_{1}, \psi\right)$, it is not clear whether equilibria where 'as much as possible is transferred to the first time period' are Pareto optimal. Another interesting point to consider is whether the above mentioned Nash equilibria are strong Nash equilibria. A strong Nash equilibrium is immune to all coalitional deviations and is defined as a strategy combination $\alpha^{*} \in \mathscr{S}_{N}$, such that for all coalitions $M \subseteq N$ and all $\alpha_{M} \in \mathscr{S}_{M}$ there exists a player $i \in M$ such that $\tilde{u}_{i}\left(\alpha_{M}, \alpha_{N \backslash M}^{*}\right)<\tilde{u}_{i}\left(\alpha^{*}\right)$. With respect to strong Nash equilibria we did not find a general result.

\subsection{Concluding Remarks}

In this chapter it is assumed that players can only make individual intertemporal transfers. Kranich et al. [24] mention briefly the influence of an aggregate transfer, which they define as a transfer of payoffs of a coalition of players, $S$ with $|S| \geq 2$, which increases the worth of $S$ at time $t, v^{t}(S)$, and decreases the worth of $S$ at time $t^{\prime}, v^{t^{\prime}}(S)$. Further research could look at the role of aggregate transfers in dynamic games. Another limitation of the model is its impossibility to transfer more payoff than the worth of the individual coalition. It is only possible for an individual to borrow from his own future. However, in real life situations an individual can take out a mortgage and go bankrupt. Other research could look at a possible extension of the model developed by Filar and Petrosjan [16] to include transfers. 



\section{5}

\section{Non-Cooperative Solutions for Claims Problems}

\subsection{Introduction}

In a claims problem - also called bankruptcy or rationing problem - a finite number of players have a claim on an estate, and the sum of these claims exceeds the estate. The question is how to distribute the estate among the players, based on their claims. Some applications of claims problems are the distribution of an inheritance among a number of heirs, liquidation of the assets of a bankrupt firm to its creditors or sharing the cost of a public facility. There is a substantial literature on this problem, starting with O'Neill [29] and Aumann and Maschler [5]. Most of this literature considers claims problems from a cooperative, axiomatic point of view. See Thomson [35] for a survey on this topic.

In this chapter ${ }^{1}$ we take a noncooperative approach to claims problems. Every player has a claim given by the claims problem, which he can split up in finitely many parts, i.e. subclaims. The estate $E$ is represented by the interval $[0, E]$, on which players can place these subclaims. Each subinterval of $[0, E]$ is distributed among the players who put claims on it, proportionally with respect to the number of claims. We assume that players know the size of the estate and each others' claims. Moreover, players' preferences are homogenous over the whole estate. This means that a player does not prefer one subinterval over another on the estate $[0, E]$. Furthermore, every partition of the estate is possible, i.e. the estate can be divided in any possible way.

The described model was already proposed by O'Neill [29] for restricted

\footnotetext{
${ }^{1}$ This chapter is based on Atlamaz, Berden, Peters and Vermeulen [4].
} 
claims problems where claims do not exceed the estate and claim height, i.e. the height of subclaims placed on intervals, equal to 1 . We extend O'Neill's model by allowing each player to put more than one claim on the same subinterval. Obviously, we cannot but allow for multiple claims, if a player's claim exceeds the estate. A model where multiple claims are possible is the following variation on Hotelling (cf. [20]). Think of the interval $[0, E]$ as a continuum of uniformly distributed consumers, and of the claimants as firms who provide services to these consumers, with firm $i$ providing total service $c_{i}$. In this case, each part of the consumer continuum is distributed proportionally with respect to the subclaims - called investments - of the firms. This interpretation naturally allows for competitive investments by different firms in one and the same consumer segment, thus, for multiple claims.

We characterize all Nash equilibria of our game and the associated payoff distributions for claims problems in which individual claims do not exceed the estate. The set of Nash equilibria resulting from O'Neill's model [29] is contained in our larger set of Nash equilibria. In the extra equilibria players claim particular parts of the estate twice. Only players with a large enough claim can claim parts of the estate twice. Roughly, these equilibria are advantageous for larger claimants. Similar to O'Neill we find that in the two player case the characterization is equal to the extended Ibn Ezra rule (O'Neill [29]) better known as the minimal overlap rule. This rule ensures that the part of the estate claimed by exactly one player is maximal, and given this, the part of the estate claimed by two players is maximal, and so on.

In general we observe that claims problems may fail to have Nash equilibria. We show that Nash equilibrium payoffs converge to the proportional distribution of the estate with respect to the claims as these claims become large.

In our basic model we assume, like O'Neill [29], that the claim height is equal to 1 . We investigate a variation on this model were claim height is varied. More specific, we look at what happens if we allow smaller claim heights and thus 'finer' claims. Not surprisingly, Nash equilibrium distributions converge to the proportional distribution as the claim height becomes smaller. In variation 4 we drop the assumption that players split their claims into finitely many parts. We assume that a strategy of a player is a (continuous) nonnegative function on the estate $[0, E]$ with total integral equal to $c_{i}$. We show that the Nash equilibrium distribution again equals the proportional distribution. We show that this is also the case under more restrictive strategies. 
Besides O'Neill [29] there are other articles which deal with strategic models imposed on claims problems. The model described in Atlamaz [3] is similar to our basic model. However, his analysis is complementary to ours. He does not give a full characterization of Nash equilibria and the associated payoff distributions for restricted claims problems and from his analysis this characterization cannot easily be deducted. Instead, he characterizes Nash equilibria of strategy profiles with exactly two claims difference between minimally and maximally claimed intervals. Chun [12] defines a game where players propose division rules. The non-cooperative solution of this game of rules converges to the outcome associated with the constrained equal awards rule. Dagan et al. [14] construct a consistency based noncooperative model that supports monotonic consistent cooperative solution concepts. Corchón and Herrero [13] define a game where players propose awards vectors that are bounded by claims. García-Jurado et al. [17] define an elementary game which has a unique payoff vector in Nash equilibrium which is associated with any acceptable bankruptcy rule.

Chapter 5 is organized as follows. In Section 5.2 the basic model is explained. Section 5.3 derives general conditions for Nash equilibria. In Section 5.4 we consider restricted claims problems and characterize Nash equilibria. In Section 5.5 we look at general (unrestricted) claims problems. A number of variation on our basic model are discussed in Section 5.6. Finally, Section 5.7 concludes.

\subsection{Preliminaries}

A claims problem is a pair $(E, \mathbf{c})$, where $E \in \mathbb{R}_{+}=\{x \in \mathbb{R} \mid x \geq 0\}$ is the amount to be divided between all players in player set $N:=\{1, \ldots, n\}$ with $n \geq 2$ and the vector $\mathbf{c}=\left(c_{1}, \ldots, c_{n}\right) \in \mathbb{R}_{+}^{N}$ with $\sum_{i=1}^{n} c_{i} \geq E$ contains the claims of all players $i \in N$.

Definition 5. A strategy of player $i$ on $(E, \mathbf{c})$ is a triple $\left(\mathbf{x}_{i}, \alpha_{i}, m_{i}\right)$ with

(i) $m_{i} \in \mathbb{N}$.

(ii) $\mathbf{x}_{i}=\left(x_{i}(0), \ldots, x_{i}\left(m_{i}\right)\right) \in \mathbb{R}^{m_{i}+1}$ with $0=x_{i}(0)<x_{i}(1)<\ldots<x_{i}\left(m_{i}\right)=E$.

(iii) $\alpha_{i}=\left(\alpha_{i}(1), \ldots, \alpha_{i}\left(m_{i}\right)\right) \in(\mathbb{N} \cup\{0\})^{m_{i}}$ such that

$$
c_{i}=\sum_{t=1}^{m_{i}} \alpha_{i}(t)\left(x_{i}(t)-x_{i}(t-1)\right) \text {. }
$$


In other words, $\mathbf{x}_{i}$ is player $i$ 's partition of the interval $[0, E]$ into $m_{i}$ subintervals and the vector $\alpha_{i}$ specifies the number of claims he puts on each subinterval such that the total amount claimed equals his claim $c_{i}$. The strategy set is the set of strategies.

A strategy profile consists of a strategy from the strategy set of every player. The payoff vector, $\left(y_{1}, \ldots, y_{n}\right) \in \mathbb{R}^{N}$, is defined over the common refinement $\mathbf{x}$ of all individual player partitions $\mathbf{x}_{i}$. Each interval generated by the common refinement is distributed among the players proportionally with respect to their claims. Given strategy profile $\left(\mathbf{x}, \alpha_{i}, m\right)_{i \in N}$ let $M=\{1, \ldots, m\}$ and let $\alpha_{N}(t)=\sum_{i \in N} \alpha_{i}(t)$ and $\alpha_{N \backslash i}(t)=\sum_{j \in N \backslash i} \alpha_{j}(t)$ for each $t \in M{ }^{2}$ Player $i$ 's payoff is defined as

$$
y_{i}=\sum_{t \in M: \alpha_{N}(t) \neq 0} \frac{\alpha_{i}(t)}{\alpha_{N}(t)}(x(t)-x(t-1))
$$

Clearly, total payoff $\sum_{i \in N} y_{i} \leq E$ for all strategy profiles. Strategy profiles where part of $[0, E]$ is unclaimed, have a total payoff which is less than the estate $E$. In Nash equilibrium there is no part of $[0, E]$ unclaimed. To see this, suppose that there is an unclaimed part in equilibrium. Since $\sum_{i \in N} c_{i} \geq E$ there exists another part of $[0, E]$ claimed at least twice. But then a player with claims in this part can gain more payoff by moving (part of) his claim to the unclaimed part.

Calculating the payoff is straightforward in case every player $i \in N$ has a strategy where his individual partition $\mathbf{x}_{i}$ is equal to the common partition $\mathbf{x}$. In general players' individual partitions $\mathbf{x}_{1}$ till $\mathbf{x}_{n}$ in a strategy profile will not be similar to the common partition $\mathbf{x}$. However, to calculate each player's payoff we need the common partition and each player's claims on every interval generated by the common partition. Hence, although in a strategy profile each player has his own individual partition, each player has an equivalent strategy containing the common refinement of all individual's partitions. Formally, $\left(\mathbf{x}, \beta_{i}, m\right)_{i \in N}$ is equivalent to $\left(\mathbf{x}_{i}, \alpha_{i}, m_{i}\right)_{i \in N}$, where $\mathbf{x}$ is the common refinement of partitions $\mathbf{x}_{i}$ for all $i \in N$ and $\beta_{i}(t)=\alpha_{i}\left(t_{i}\right)$ for all $t \in\{1, \ldots, m\}$ and all $t_{i} \in\left\{1, \ldots, m_{i}\right\}$ with $[x(t-1), x(t)] \subseteq\left[x_{i}\left(t_{i}-1\right), x_{i}\left(t_{i}\right)\right]$ for all $i \in N$. It is without loss of generality to assume in the remainder of this chapter that the strategies of all players have the same partition. The next example illustrates the introduced concepts.

\footnotetext{
${ }^{2}$ A coalition is written without curly braces, e.g. $\mathrm{i} j$ is written instead of $\{i, j\}$ for $i, j \in N$.
} 
Example 13. Consider claims problem $\left(E\right.$,c) with $E=10, n=2$ and $\mathbf{c}=\left(c_{1}, c_{2}\right)=$ $(5,8)$. Suppose player 1's strategy $\left(\mathbf{x}_{1}, \alpha_{1}, m_{1}\right)$ with $\mathbf{x}_{1}=(0,5,10), \alpha_{1}=(1,0)$ and $m_{1}=2$. The partition $\mathbf{x}_{1}$ divides the interval $[0, E]=[0,10]$ into $m_{1}=2$ subintervals, namely $[0,5]$ and $[5,10]$ on which player 1 puts respectively 1 and 0 claims. In total player 1 places $1 \cdot(5-0)+0 \cdot(10-5)=5$ which is exactly the size of his claim $c_{1}$ in the claims problem. Suppose player 2's strategy $\left(\mathbf{x}_{2}, \alpha_{2}, m_{2}\right)$ with $\mathbf{x}_{2}=(0,2,6,10), \alpha_{2}=(2,1,0)$ and $m_{2}=3$. The partition $\mathbf{x}_{2}$ creates $m_{2}=3$ subintervals, $[0,2],[2,6]$ and $[6,10]$ on which player 2 puts respectively 2,1 and 0 claims. In total player 2 places $2 \cdot(2-0)+1 \cdot(6-2)+0 \cdot(10-6)=8$, which is exactly $c_{2}$. As can be verified, the common refinement of partitions $\mathbf{x}_{1}$ and $\mathbf{x}_{2}$ generates subintervals $[0,2],[2,5],[5,6]$ and $[6,10]$ and is denoted by $\mathbf{x}=(0,2,5,6,10)$. It is easy to see that player 1 has respectively $1,1,0$ and 0 claims on the subintervals generated by $\mathbf{x}$, and his strategy can equivalently be written as $(\mathbf{x},(1,1,0,0), 4)$. Player 2 has respectively $2,1,1$ and 0 claims on the subintervals generated by $\mathbf{x}$, similar to strategy $(\mathbf{x},(2,1,1,0), 4)$. Player 1's payoff equals $y_{1}=\frac{1}{1+2} \cdot(2-0)+\frac{1}{1+1} \cdot(5-2)+\frac{0}{0+1} \cdot(6-5)=2 \frac{1}{6}$. In the same way we find that player 2's payoff equals $y_{2}=3 \frac{5}{6}$.

A strategy profile is a Nash equilibrium if no player can increase his payoff by reshuffling his claims. Intuitively, this means that the marginal utilities on the intervals in the strategy profile should be 'more or less' equal: complete equality is generally not possible since the claims are integers. For simplicity, we will just define Nash equilibrium strategy profiles in terms of marginal utilities or, more specifically, marginal gains and losses. A useful tool in this analysis is the marginal gain table of which a part is shown in the Appendix. The element in row number $r$ and column number $c$ contains the outcome of the function $u: \mathbb{N} \times \mathbb{N} \rightarrow \mathbb{R}$ which is defined for $r \geq 1$ and $c \geq 0$ as

$$
u(r, c)= \begin{cases}1 & \text { if } c=0 \text { and } r=1, \\ \frac{r}{r+c}-\frac{r-1}{r+c-1} & \text { otherwise. }\end{cases}
$$

The element $u(r, c)$ represents player $i$ 's marginal gain incurred on an interval of length 1 from claiming it $r$ instead of $r-1$ times, given that it is claimed $c$ times by all other players.

Theorem 7. Strategy profile $\left(\mathbf{x}, \alpha_{i}, m\right)_{i \in N}$ is a Nash equilibrium for claims problem $(E, \mathbf{c})$ if and only if $\alpha_{N}(t) \geq 1$ for all $t \in M$ and the following condition is satisfied: for all $t, t^{\prime} \in M, t \neq t^{\prime}$ and all $i \in N$, if $\alpha_{i}(t) \geq 1$ and $\alpha_{N}(t)>1$, then

$$
\frac{\alpha_{i}(t)}{\alpha_{N}(t)}-\frac{\alpha_{i}(t)-1}{\alpha_{N}(t)-1} \geq \frac{\alpha_{i}\left(t^{\prime}\right)+1}{\alpha_{N}\left(t^{\prime}\right)+1}-\frac{\alpha_{i}\left(t^{\prime}\right)}{\alpha_{N}\left(t^{\prime}\right)}
$$


Inequality (5.2) says that the loss incurred by taking away a claim (or part of a claim) from some interval $t$ should be at least as big as the gain obtained from placing that claim on some other interval $t^{\prime}$. The marginal gain table can be used to check condition (5.2); the left hand side of (5.2) is $u\left(\alpha_{i}(t), \alpha_{N \backslash i}(t)\right)$, the right hand side is $u\left(\alpha_{i}\left(t^{\prime}\right)+1, \alpha_{N \backslash i}\left(t^{\prime}\right)\right)$. A particular consequence of inequality (5.2) is that, if in equilibrium $\alpha_{i}(t)=\alpha_{N}(t)$ for some $i \in N$ and $t \in M$, then $\alpha_{i}(t)=\alpha_{N}(t)=1$, because there is always a $t^{\prime} \in M$ with $\alpha_{i}\left(t^{\prime}\right)<\alpha_{N}\left(t^{\prime}\right)$. This means that if a player is the only one to claim a certain part of the estate, then he claims this part only once.

The proof of Theorem 7 is given in the Appendix. In the Appendix we use the term 'elementary change'. We define an elementary change of $\alpha_{i}$ as follows: there exist $t, t^{\prime} \in M$ for which $\alpha_{i}^{\prime}(t)=\alpha_{i}(t)-1, \alpha_{i}^{\prime}\left(t^{\prime}\right)=\alpha_{i}\left(t^{\prime}\right)+1$ and $\alpha_{i}^{\prime}(s)=\alpha_{i}(s)$ for all $s \neq t, t^{\prime}$. Hence, an elementary change only considers a shift of one claim from (part of) interval $t$ to (part of) interval $t^{\prime}$.

A restricted claims problem is a claims problem $(E, \mathbf{c})$ with $c_{i} \leq E$ for all $i \in N$. O'Neill [29] considered restricted claims problems and a noncooperative claims game in which each part can be claimed at most once by the same player. We will see that allowing players to claim parts more than once leads to a larger set of Nash equilibria and a larger set of according payoff vectors. Of course, for general claim problems it is not avoidable to allow multiple claims.

\subsection{Some general results}

In Lemma 11- 14 strategy profile $\left(\mathbf{x}, \alpha_{i}, m\right)_{i \in N}$ is a Nash equilibrium for claims problem $(E, \mathbf{c})$. Let $M=\{1, \ldots, m\}$ and denote $\alpha_{\max }=\max _{s \in M} \alpha_{N}(s)$ and $\alpha_{\min }=$ $\min _{s \in M} \alpha_{N}(s)$. We derive results, which have independent interest and will be used for later analysis.

The first lemma says that if a player has at least one claim on interval $t$, then he has to have at least one claim on all intervals with total amount of claims less than or equal to the total amount of claims on interval $t$ minus two claims. It will become clear from Lemma 16 that the situation described in Lemma 11 does never occur in case Nash equilibria in restricted claims problems are considered.

Lemma 11. Let $i \in N, t, t^{\prime} \in M, \alpha_{i}(t) \geq 1$ and $\alpha_{N}(t)-\alpha_{N}\left(t^{\prime}\right) \geq 2$, then $\alpha_{i}\left(t^{\prime}\right)>0$. 
Proof. Suppose, contrary to what we want to prove, that $\alpha_{i}\left(t^{\prime}\right)=0$. Then

$$
\frac{\alpha_{i}\left(t^{\prime}\right)+1}{\alpha_{N}\left(t^{\prime}\right)+1}=\frac{1}{\alpha_{N}\left(t^{\prime}\right)+1}>\frac{1}{\alpha_{N}(t)} \geq \frac{\alpha_{i}(t)}{\alpha_{N}(t)}-\frac{\alpha_{i}(t)-1}{\alpha_{N}(t)-1}
$$

where the strict inequality follows from $\alpha_{N}\left(t^{\prime}\right) \leq \alpha_{N}(t)-2$. This contradicts condition (5.2) in Theorem 7. Thus, $\alpha_{i}\left(t^{\prime}\right)>0$.

The next lemma says that if a player has at least two claims on interval $t$, then he should put at least one claim on each interval $t^{\prime}$ with a total amount of claims that does not exceed the total amount of claims on interval $t$.

Lemma 12. Let $i \in N, t, t^{\prime} \in M, \alpha_{i}(t) \geq 2$ and $\alpha_{N}\left(t^{\prime}\right) \leq \alpha_{N}(t)$, then $\alpha_{i}\left(t^{\prime}\right)>0$.

Proof. Suppose contrary to what we want to prove that $\alpha_{i}\left(t^{\prime}\right)=0$. Then

$$
\begin{aligned}
\frac{\alpha_{i}\left(t^{\prime}\right)+1}{\alpha_{N}\left(t^{\prime}\right)+1}-\frac{\alpha_{i}\left(t^{\prime}\right)}{\alpha_{N}\left(t^{\prime}\right)} & =\frac{1}{\alpha_{N}\left(t^{\prime}\right)+1} \quad \geq \quad \frac{1}{\alpha_{N}(t)+1} \\
& >\frac{2}{\alpha_{N}(t)}-\frac{1}{\alpha_{N}(t)-1}=\frac{\alpha_{N}(t)-2}{\alpha_{N}(t)\left(\alpha_{N}(t)-1\right)} \\
& \geq \frac{\alpha_{N}(t)-\alpha_{i}(t)}{\alpha_{N}(t)\left(\alpha_{N}(t)-1\right)}=\frac{\alpha_{i}(t)}{\alpha_{N}(t)}-\frac{\alpha_{i}(t)-1}{\alpha_{N}(t)-1},
\end{aligned}
$$

where the strict inequality follows from $\frac{1}{\alpha_{N}(t)}<\frac{1}{2} \cdot \frac{1}{\alpha_{N}(t)-1}+\frac{1}{2} \cdot \frac{1}{\alpha_{N}(t)+1} \cdot 3$ This contradicts condition (5.2) in Theorem 7. Thus, $\alpha_{i}\left(t^{\prime}\right)>0$.

In the following lemma it is shown that maximally claimed intervals contain roughly at most the squared amount of claims of minimally claimed intervals.

Lemma 13. $\alpha_{\max } \leq \alpha_{\min }\left(\alpha_{\min }+1\right)$.

Proof. Let $t, t^{\prime} \in M$ and $\alpha_{N}(t)=\alpha_{\max }, \alpha_{i}(t) \geq 1$ and $\alpha_{N}\left(t^{\prime}\right)=\alpha_{\min }$. Because $\sum_{i \in N} c_{i} \geq E$ and $\alpha_{N}(s) \geq 1$ for all $s \in M$, there are two cases to consider. If $\alpha_{\max }=$ 1 , then we are done. If $\alpha_{\max }>1$, then from condition (5.2) in Theorem 7 it follows that

$$
\frac{\alpha_{\max }-\alpha_{i}(t)}{\alpha_{\max }\left(\alpha_{\max }-1\right)}=\frac{\alpha_{i}(t)}{\alpha_{N}(t)}-\frac{\alpha_{i}(t)-1}{\alpha_{N}(t)-1} \geq \frac{\alpha_{i}\left(t^{\prime}\right)+1}{\alpha_{N}\left(t^{\prime}\right)+1}-\frac{\alpha_{i}\left(t^{\prime}\right)}{\alpha_{N}\left(t^{\prime}\right)}=\frac{\alpha_{\min }-\alpha_{i}\left(t^{\prime}\right)}{\alpha_{\min }\left(\alpha_{\min }+1\right)} .
$$

In Nash equilibrium $\alpha_{\min }-\alpha_{i}\left(t^{\prime}\right) \geq 1$. To see this, suppose $\alpha_{\min }-\alpha_{i}\left(t^{\prime}\right)=0$, then as was noted before $\alpha_{\min }=\alpha_{i}\left(t^{\prime}\right)=1$. In case $\alpha_{\max }>2$, there exists a player $j \in N \backslash i$ who can improve by shifting one claim from $\alpha_{\max }$ onto $\alpha_{\min }$.

\footnotetext{
${ }^{3}$ By strict convexity of the function $x \rightarrow \frac{1}{x}$
} 
In case $\alpha_{\max }=2$, a player $j \in N \backslash i$ does not gain nor loose from shifting claims from $\alpha_{\max }$ onto $\alpha_{\min }$. Then, since $\alpha_{i}(t) \geq 1$, it follows that

$$
\frac{1}{\alpha_{\max }} \geq \frac{\alpha_{\max }-1}{\alpha_{\max }\left(\alpha_{\max }-1\right)} \geq \frac{\alpha_{\max }-\alpha_{i}(t)}{\alpha_{\max }\left(\alpha_{\max }-1\right)} \geq \frac{\alpha_{\min }-\alpha_{i}\left(t^{\prime}\right)}{\alpha_{\min }\left(\alpha_{\min }+1\right)} \geq \frac{1}{\alpha_{\min }\left(\alpha_{\min }+1\right)} .
$$

Thus, we have shown that $\alpha_{\max } \leq \alpha_{\min }\left(\alpha_{\min }+1\right)$.

The next lemma shows that if each player claims the estate at least once, then the difference in claims between the maximally claimed interval and the minimally claimed interval is less than or equal to two claims.

Lemma 14. If $\alpha_{i}(t) \geq 1$ for all $i \in N$ and all $t \in M$, then $\alpha_{\max }-\alpha_{\min } \leq 2$.

Proof. Let $\alpha_{i}(s) \geq 1$ for all $i \in N$ and $s \in M$. From condition (5.2) in Theorem 7 it follows that for all $i \in N$, all $t, t^{\prime} \in M, \alpha_{i}(t) \geq 1$ and $\alpha_{N}(t)>1$ we have

$$
\frac{\alpha_{N}(t)-\alpha_{i}(t)}{\alpha_{N}(t)\left(\alpha_{N}(t)-1\right)}=\frac{\alpha_{i}(t)}{\alpha_{N}(t)}-\frac{\alpha_{i}(t)-1}{\alpha_{N}(t)-1} \geq \frac{\alpha_{i}\left(t^{\prime}\right)}{\alpha_{N}\left(t^{\prime}\right)+1}-\frac{\alpha_{i}\left(t^{\prime}\right)}{\alpha_{N}\left(t^{\prime}\right)}=\frac{\alpha_{N}\left(t^{\prime}\right)-\alpha_{i}\left(t^{\prime}\right)}{\alpha_{N}\left(t^{\prime}\right)\left(\alpha_{N}\left(t^{\prime}\right)+1\right)} .
$$

Because this holds for all $i \in N$, it is also true that

$$
\sum_{i \in N} \frac{\alpha_{N}(t)-\alpha_{i}(t)}{\alpha_{N}(t)\left(\alpha_{N}(t)-1\right)} \geq \sum_{i \in N} \frac{\alpha_{N}\left(t^{\prime}\right)-\alpha_{i}\left(t^{\prime}\right)}{\alpha_{N}\left(t^{\prime}\right)\left(\alpha_{N}\left(t^{\prime}\right)+1\right)} .
$$

Since $\alpha_{i}(s) \geq 1$ for all $i \in N$ and all $s \in M$ it follows that

$$
\frac{1}{\alpha_{N}(t)-1}=\frac{n \cdot \alpha_{N}(t)-\alpha_{N}(t)}{\alpha_{N}(t)\left(\alpha_{N}(t)-1\right)} \geq \frac{n \cdot \alpha_{N}\left(t^{\prime}\right)-\alpha_{N}\left(t^{\prime}\right)}{\alpha_{N}\left(t^{\prime}\right)\left(\alpha_{N}\left(t^{\prime}\right)+1\right)}=\frac{1}{\alpha_{N}\left(t^{\prime}\right)+1} .
$$

Hence, $\alpha_{N}(t)-\alpha_{N}\left(t^{\prime}\right) \leq 2$. The result holds for arbitrary $t, t^{\prime} \in M$.

In Lemma 15 it is shown that independent of other players' strategies an individual can always play a strategy such that he receives a payoff which is at least equal to the division according to the proportional rule minus an error term. Given claims problem $(E, \mathbf{c})$, the proportional rule distributes the estate $E$ proportional to the claims $\mathbf{c}$ and assigns the payoff vector $E \mathbf{c} / \sum_{i \in N} c_{i}$.

Lemma 15. Let $(E, \mathbf{c})$ be a claims problem. Let $\left(\mathbf{x}, \alpha_{j}, m\right)_{j \in N \backslash i}$ be any strategy profile of players $N \backslash i$. Player $i$ can play a strategy against $\left(\mathbf{x}, \alpha_{j}, m\right)_{j \in N \backslash i}$ which generates him a payoff of at least $\left(c_{i} / \sum_{j \in N} c_{j}-1 / \min _{s \in M} \alpha_{N \backslash i}(s)\right) E$.

Proof. Consider any strategy profile restricted to $N \backslash i$, namely $\left(\mathbf{x}, \alpha_{j}, m\right)_{j \in N \backslash i}$. Let $M=\{1, \ldots, m\}$. Assume that $\sum_{j \in N \backslash i} \alpha_{j}(t)>0$ for all $t \in M{ }^{4}$

\footnotetext{
${ }^{4}$ If this assumption is not satisfied, then player $i$ can even do better by putting a claim of very small height on the non-claimed part(s) of the interval $[0, E]$.
} 
Take $s_{i}=c_{i} / \sum_{j \in N \backslash i} c_{j}$ and define for all $t \in M^{5}$

$$
n_{i}(t)=\left(s_{i} \cdot \alpha_{N \backslash i}(t)-\left\lfloor s_{i} \cdot \alpha_{N \backslash i}(t)\right\rfloor\right)(x(t)-x(t-1)) .
$$

We will show that player i can guarantee a payoff of at least $E c_{i} / \sum_{j \in N} c_{j}-$ $E / \min _{s \in M} \alpha_{N \backslash i}(s)$ by claiming for all $t \in M$

$$
\begin{aligned}
& \left\lfloor s_{i} \cdot \alpha_{N \backslash i}(t)\right\rfloor+1 \text { times interval }\left[x(t-1), x(t-1)+n_{i}(t)\right], \text { and } \\
& \left\lfloor s_{i} \cdot \alpha_{N \backslash i}(t)\right\rfloor \text { times interval }\left[x(t-1)+n_{i}(t), x(t)\right] .
\end{aligned}
$$

This way player $i$ places in total

$$
\begin{aligned}
\sum_{t \in M}\left(\left\lfloor s_{i} \cdot \alpha_{N \backslash i}(t)\right\rfloor+1\right) n_{i}(t)+\sum_{t \in M}\left\lfloor s_{i} \cdot \alpha_{N \backslash i}(t)\right\rfloor\left(x(t)-x(t-1)-n_{i}(t)\right) \\
=\sum_{t \in M}\left\lfloor s_{i} \cdot \alpha_{N \backslash i}(t)\right\rfloor(x(t)-x(t-1))+\sum_{t \in M} n_{i}(t) \\
=\sum_{t \in M} s_{i} \cdot \alpha_{N \backslash i}(t)(x(t)-x(t-1))=s_{i}\left(\sum_{j \in N} c_{j}-c_{i}\right)=c_{i},
\end{aligned}
$$

and according to (5.1) he receives payoff

$$
\begin{aligned}
y_{i}=\sum_{t \in M} \frac{\left\lfloor s_{i} \cdot \alpha_{N \backslash i}(t)\right\rfloor+1}{\left\lfloor\left(c_{i} / \sum_{j \in N \backslash i} c_{j}\right) \cdot \alpha_{N \backslash i}(t)\right\rfloor+1+\alpha_{N \backslash i}(t)} n_{i}(t) \\
\quad+\sum_{t \in M} \frac{\left\lfloor s_{i} \cdot \alpha_{N \backslash i}(t)\right\rfloor}{\left\lfloor s_{i} \cdot \alpha_{N \backslash i}(t)\right\rfloor+\alpha_{N \backslash i}(t)}\left(x(t)-x(t-1)-n_{i}(t)\right) .
\end{aligned}
$$

Define $\underline{G}_{i}$ and $\bar{G}_{i}$ as follows,

$$
\begin{aligned}
\underline{G}_{i} & =\sum_{t \in M} \frac{\left\lfloor s_{i} \cdot \alpha_{N \backslash i}(t)\right\rfloor}{\left\lfloor s_{i} \cdot \alpha_{N \backslash i}(t)\right\rfloor+\alpha_{N \backslash i}(t)}(x(t)-x(t-1)) \\
\bar{G}_{i} & =\sum_{t \in M} \frac{\left\lfloor s_{i} \cdot \alpha_{N \backslash i}(t)\right\rfloor+1}{\left\lfloor s_{i} \cdot \alpha_{N \backslash i}(t)\right\rfloor+1+\alpha_{N \backslash i}(t)}(x(t)-x(t-1)) .
\end{aligned}
$$

It holds that $\underline{G}_{i} \leq y_{i}<\bar{G}_{i}$. The first inequality follows from $n_{i}(t) \geq 0$. The second inequality follows from $n_{i}(t)<x(t)-x(t-1)$.

Suppose it were possible for player $i$ to claim $\alpha_{i}(t)=s_{i} \cdot \alpha_{N \backslash i}(t)$ times interval $[x(t-1), x(t)]$ for all $t \in M$. As can easily be verified, he then claims in total $c_{i}$ and according to (5.1) receives $y_{i}^{*}=E c_{i} / \sum_{j \in N} c_{j}$.

It holds that $\underline{G}_{i} \leq y_{i}^{*}<\bar{G}_{i}$. The first inequality follows from $\left\lfloor s_{i} \cdot \alpha_{N \backslash i}(t)\right\rfloor \leq$ $s_{i} \cdot \alpha_{N \backslash i}(t)$. The second inequality follows from $s_{i} \cdot \alpha_{N \backslash i}(t)<\left\lfloor s_{i} \cdot \alpha_{N \backslash i}(t)\right\rfloor+1$.

\footnotetext{
${ }^{5} \mathrm{By}\lfloor x\rfloor$ we denote the entier of $x$ and is defined as follows $\lfloor x\rfloor:=\max \{z \in \mathbb{Z} \mid z \leq x\}$.
} 
Moreover,

$$
\begin{aligned}
\bar{G}_{i} & <\underline{G}_{i}+\sum_{t \in M} \frac{1}{\left\lfloor s_{i} \cdot \alpha_{N \backslash i}(t)\right\rfloor+1+\alpha_{N \backslash i}(t)}(x(t)-x(t-1)) \\
& <\underline{G}_{i}+\frac{1}{\min _{s \in M} \alpha_{N \backslash i}(s)} E .
\end{aligned}
$$

As a result $\underline{G}_{i} \leq y_{i}, y_{i}^{*}<\bar{G}_{i}<\underline{G}_{i}+E / \min _{s \in M} \alpha_{N \backslash i}(s)$, which means that

$$
\left|y_{i}-y_{i}^{*}\right|=\left|y_{i}-\frac{c_{i}}{\sum_{j \in N} c_{j}} E\right|<\frac{1}{\min _{s \in M} \alpha_{N \backslash i}(s)} E .
$$

Player $i$ can play a strategy against $\left(\mathbf{x}, \alpha_{j}, m\right)_{j \in N \backslash i}$ such that he receives at least

$$
\left(\frac{c_{i}}{\sum_{j \in N} c_{j}}-\frac{1}{\min _{s \in M} \alpha_{N \backslash i}(s)}\right) E
$$

\subsection{Restricted claims problems}

We first derive some additional results for Nash equilibria in restricted claims problems, and next use these to characterize all Nash equilibria in such problems.

In Lemma 16-19 strategy profile $\left(\mathbf{x}, \alpha_{i}, m\right)_{i \in N}$ is a Nash equilibrium for the restricted claims problem $(E, \mathbf{c})$.

The first lemma shows that each player has at most one claim in the maximally claimed intervals.

Lemma 16. $\alpha_{i}(t) \leq 1$ for all $i \in N$ and all $t \in M$ with $\alpha_{N}(t)=\alpha_{\max }$.

Proof. Suppose, contrary to what we wish to prove, that $\alpha_{i}(t) \geq 2$ for some $i \in N$ and $t \in M$ with $\alpha_{N}(t)=\alpha_{\max }$. Since $c_{i} \leq E$, there must be a $t^{\prime} \in M$ with $\alpha_{i}\left(t^{\prime}\right)=$ 0 , but this contradicts Lemma 12 .

The next lemma says that the differences in total numbers of claims between intervals can be at most 1 .

Lemma 17. $\alpha_{\max }-\alpha_{\min } \leq 1$. 
Proof. Suppose, contrary to what we want to show, that there are $t, t^{\prime} \in M$ with $\alpha_{N}(t)-\alpha_{N}\left(t^{\prime}\right)>1, \alpha_{N}(t)=\alpha_{\max }, \alpha_{N}\left(t^{\prime}\right)=\alpha_{\min }$. Assume $\alpha_{N}(t)-\alpha_{N}\left(t^{\prime}\right)=2$. Lemma 16 implies that there are exactly $\alpha_{N}(t)$ different players with a claim on $t$. By Lemma 11 all these players have at least one claim on $t^{\prime}$, but this is clearly impossible.

In Lemma 18 it is shown that each player has at most two claims on each interval.

Lemma 18. $\alpha_{i}(t) \leq 2$ for all $i \in N$ and all $t \in M$.

Proof. Let $i \in N$. In view of Lemmas 16 and 17 it is sufficient to prove $\alpha_{i}(t) \leq 2$ for $t \in M$ with $\alpha_{N}(t)=\alpha_{\max }-1$. Suppose, to the contrary, that $\alpha_{i}(t) \geq 3$ for such a $t$, and thus $\alpha_{\max } \geq 4$. Since $c_{i} \leq E$ and by Lemma 12, there is $t \in M$ with $\alpha_{i}\left(t^{\prime}\right)=0$ and $\alpha_{N}\left(t^{\prime}\right)=\alpha_{\text {max }}$. By using condition (5.2) in Theorem 7 we obtain

$$
\frac{\left(\alpha_{\max }-1\right)-\alpha_{i}(t)}{\left(\alpha_{\max }-1\right)\left(\alpha_{\max }-2\right)} \geq \frac{1}{\alpha_{\max }+1}
$$

hence

$$
\frac{\alpha_{\max }-4}{\left(\alpha_{\max }-1\right)\left(\alpha_{\max }-2\right)} \geq \frac{1}{\alpha_{\max }+1} .
$$

It is easy to check that the latter inequality cannot hold.

Finally, Lemma 19 shows that if an interval is claimed twice by a player, then there must be an interval with at least five claims on it. Consequently, by Lemma 16, the number of players is at least five as well.

Lemma 19. Let $i \in N$ and $\alpha_{i}(t)=2$ for some $t \in M$ with $\alpha_{N}(t)=\alpha_{\max }-1$. Then $\alpha_{\max } \geq 5$.

Proof. As in the proof of Lemma 18, there is a $t^{\prime} \in M$ with $\alpha_{i}\left(t^{\prime}\right)=0$ and $\alpha_{N}\left(t^{\prime}\right)=$ $\alpha_{\max }$. By condition (5.2) in Theorem 7,

$$
\frac{2}{\alpha_{\max }-1}-\frac{1}{\alpha_{\max }-2} \geq \frac{1}{\alpha_{\max }+1} .
$$

which implies $\alpha_{\max } \geq 5$.

Lemmas 12 and 16-19 provide a number of necessary conditions for a strategy profile $\left(\mathbf{x}, \alpha_{i}, m\right)_{i \in N}$ to be a Nash equilibrium. By Lemma 16 in particular it follows that there is a $K \in \mathbb{N}$ such that on each interval there are $K$ or $K+1$ 
claims. Let $R \in \mathbb{R}$ be the total part of $E$ that is claimed $K+1$ times. Then $0 \leq R<E$ and we must have

$$
\sum_{i \in N} c_{i}=K(E-R)+(K+1) R=K \cdot E+R .
$$

This means that $K$ and $R$ are uniquely determined by $E$ and $\sum_{i \in N} c_{i}$. The main result of this section is the following theorem, which says that the necessary conditions provided by the mentioned lemmas are also sufficient.

Theorem 8. Let $(E, \mathbf{c})$ be a restricted claims problem, and let $K \in \mathbb{N}$ and $R \in \mathbb{R}$ with $0 \leq R<E$ be the unique numbers satisfying $\sum_{i \in N} c_{i}=K \cdot E+R$. Then $a$ strategy profile $\left(\mathbf{x}, \alpha_{i}, m\right)_{i \in N}$ for $(E, \mathbf{c})$ is a Nash equilibrium if and only if the following four conditions are satisfied:

(A1) For all $t \in M, \alpha_{N}(t) \in\{K, K+1\}$.

(A2) For all $t \in M$ and $i \in N$, if $\alpha_{N}(t)=K+1$ then $\alpha_{i}(t) \leq 1$.

(A3) For all $t \in M$ and $i \in N$, if $\alpha_{N}(t)=K$ then $\alpha_{i}(t) \leq 2$.

(A4) For all $t \in M$ and $i \in N$, if $\alpha_{i}(t)=2$ then $\alpha_{i}\left(t^{\prime}\right) \geq 1$ for all $t^{\prime} \in M$ with $\alpha_{N}\left(t^{\prime}\right)=K$, and $K \geq 4$.

Proof. Necessity follows from Lemmas 12 till 19. For sufficiency, it is convenient to consider Tables 5.1 and 5.2 associated with a claims profile satisfying (A1)-(A4).

Table 5.1: Marginal losses incurred by removing a claim from interval $t$.

\begin{tabular}{cccc} 
Case & $\alpha_{N}(t)$ & $\alpha_{i}(t)$ & marginal loss \\
\hline (i) & $K+1$ & 1 & $\frac{1}{K+1}$ \\
(ii) & $K$ & 1 & $\frac{1}{K}$ \\
(iii) & $K$ & 2 & $\frac{2}{K}-\frac{1}{K-1}$ \\
\hline
\end{tabular}

It can easily be checked that condition (5.2) in Theorem 7 is satisfied for each shift of a claim from one of the cases (i)-(iii) to one of the cases (a)-(e). Note that a shift from (iii) to (a) cannot occur by condition (A4). 
Table 5.2: Marginal gain incurred by adding a claim to interval $t^{\prime}$.

Case $\alpha_{N}\left(t^{\prime}\right) \quad \alpha_{i}\left(t^{\prime}\right) \quad$ marginal gain

\begin{tabular}{cccc}
\hline (a) & $K$ & 0 & $\frac{1}{K+1}$ \\
(b) & $K$ & 1 & $\frac{2}{K+1}-\frac{1}{K}$ \\
(c) & $K$ & 2 & $\frac{3}{K+1}-\frac{2}{K}$ \\
(d) & $K+1$ & 0 & $\frac{1}{K+2}$ \\
(e) & $K+1$ & 1 & $\frac{2}{K+2}-\frac{1}{K+1}$ \\
\hline
\end{tabular}

Theorem 8 gives a complete characterization of the Nash equilibrium strategy profiles for restricted claims problems. We conclude this section with a description of the associated payoff vectors. Let $r_{i}$ denote the part of player $i$ 's total claim $c_{i}$ invested in intervals with $K+1$ number of claims. Obviously, $r_{i} \leq c_{i}$ and by (A2) in Theorem $8, r_{i} \leq R$. Hence, $r_{i} \leq \min \left\{c_{i}, R\right\}$. On the other hand, by (A3), we have $c_{i}-r_{i} \leq 2(E-R)$ : the part invested in intervals with number of claims equal to $K$ cannot exceed two times the remaining part $E-R$ of the estate. Hence, $r_{i} \geq \max \left\{c_{i}-2(E-R), 0\right\}$. Additionally, the $r_{i}$ should sum up to $(K+1) R$. Summarizing, each Nash equilibrium corresponds to a vector $\left(r_{1}, \ldots, r_{n}\right)$ which satisfies both parts in (5.3), namely

$$
\begin{gathered}
\sum_{i \in N} r_{i}=(K+1) R \\
\max \left\{c_{i}-2(E-R), 0\right\} \leq r_{i} \leq \min \left\{c_{i}, R\right\} \text { for all } i \in N .
\end{gathered}
$$

Conversely, each such vector gives rise to a Nash equilibrium. Although there are many Nash equilibria associated with the same vector $\left(r_{1}, \ldots, r_{n}\right)-$ because there is quite some freedom in choosing the intervals and distributing the claims - all these Nash equilibria have the same payoff, which is

$$
y_{i}=\frac{r_{i}}{K+1}+\frac{c_{i}-r_{i}}{K} .
$$

To see that payoff is actually as is described by (5.4), consider two cases. (i) If $c_{i}-r_{i} \leq E-R$, then it is easy to see that player $i$ 's payoff is as given in (5.4). (ii) In case $E-R<c_{i}-r_{i} \leq 2(E-R)$, player $i$ 's payoff equals $\frac{r_{i}}{K+1}+\frac{E-R}{K}+\frac{c_{i}-r_{i}-(E-R)}{K}=$ $\frac{r_{i}}{K+1}+\frac{c_{i}-r_{i}}{K}$. Both parts of equation (5.3) and (5.4) imply that the set of payoff vectors attainable by a Nash equilibrium is determined by linear inequalities. 
These equations are also convenient to check if a certain payoff, e.g. the payoff prescribed by some well known rule, can be obtained in an equilibrium.

Example 14. Consider claims problem $(E, \mathbf{c})$ with $E=6, n=3$ and $\mathbf{c}=(1,2,5)$. The so-called proportional rule distributes the estate proportional to the claims and assigns the payoff vector $y=\left(\frac{6}{8}, \frac{12}{8}, \frac{30}{8}\right)$. For this problem, $K=1$ and $R=2$. From condition (5.3) we then find $r_{1}+r_{2}+r_{3}=4$ and $0 \leq r_{1} \leq 1,0 \leq r_{2} \leq 2$ and $0 \leq r_{2} \leq 2$. Hence, in a Nash equilibrium player 1's payoff is in $\left[\frac{1}{2}, 1\right]$, player 2's payoff is in $\left[1, \frac{3}{2}\right]$ and player 3's payoff is in $\left[4, \frac{9}{2}\right]$. Since $\frac{30}{8}<4$, it follows that the proportional payoff vector is not an equilibrium payoff vector.

Remark. If $n=2$ then it follows from the results above that the only choice for $\left(r_{1}, r_{2}\right)$ is $r_{1}=r_{2}=R$. The according unique equilibrium payoffs are $y_{i}=\frac{1}{2}(E+$ $c_{i}-c_{j}$ ) for $i=1,2$ and $j \neq i$. These payoffs coincide with the payoffs of the socalled minimal overlap rule. See Alcalde et al. [1] for a recent characterization of this rule.

We conclude this section with an example which indicates that Nash equilibria in which some players have double claims on part of the estate tend to favor 'big' players.

Example 15. Consider claims problem $(E, \mathbf{c})$ with $E=1, n=17, c_{1}=c_{2}=9$ and $c_{3}=\ldots=c_{17}=2$. Then $K=4$ and $R=8$. From the point of view of player 1 and 2 , the highest payoff generated by a Nash equilibrium is one where both players claim part $E-R$ of the estate double. Consider for example Nash equilibrium $\left(\mathbf{x}, \alpha_{i}, m\right)_{i \in N}$ with $m=7$ and $\mathbf{x}=(0,2,4,5,6,7,8,10), \alpha_{1}=\alpha_{2}=(1,1,1,0,0,0,2)$, $\alpha_{3}=\ldots=\alpha_{5}=(1,0,0,0,0,0,0), \alpha_{6}=\ldots=\alpha_{8}=(0,1,0,0,0,0,0), \alpha_{9}=\ldots=\alpha_{11}=$ $(0,0,1,1,0,0,0), \alpha_{12}=\ldots=\alpha_{14}=(0,0,0,0,1,1,0), \alpha_{15}=(0,0,0,1,1,0,0), \alpha_{16}=$ $(0,0,0,0,1,1,0)$ and $\alpha_{17}=(0,0,0,1,0,1,0)$. Then players 1 and 2 each have a payoff equal to $\frac{1}{5} \cdot 5+\frac{2}{4} \cdot 2=2$ and the other players each receive $\frac{2}{5}$. In a Nash equilibrium where players 1 and 2 do not have double claims, they receive at most $\frac{1}{5} \cdot 7+\frac{1}{4} \cdot 2=\frac{19}{10}$.

\subsection{General claims problems}

If a claims problem represents a liquidation of assets of a bankrupt firm among its creditors, it is likely that a creditor's claim exceeds the amount of liquidated 
assets. Because of this reason we consider in this section claims problems without restrictions on the claims (not all $c_{i}$ are smaller than or equal to $E$ ). Although a Nash equilibrium for restricted claims problems is always guaranteed, it may fail to exist in the more general class of claims problems.

Example 16. Consider claims problem $(E, \mathbf{c})$ with $E=1, n=2$ and $\mathbf{c}=\left(1 \frac{1}{10}, \frac{1}{2}\right)$. Assume without loss of generality that player 2 puts claims on the interval $[0, d]$ with $d \leq \frac{1}{2}$. Obviously, to obtain a Nash equilibrium, player 1 should claim $[d, 1]$ exactly once. Suppose there were a part of $[0, d]$ claimed more than once by player 2, say without loss of generality $[0, e]$ with $e \leq d$. Player 2's marginal loss on $[0, e]$ is at most $\frac{1}{6}$, attained if player 1 has one claim on $[0, e]$ and player 2 has two claims on $[0, e]$ : then 2's marginal loss is $\frac{2}{3}-\frac{1}{2}$. His marginal gain by adding a claim on $[d, 1]$ would be $\frac{1}{2}$, hence this would not be part of a Nash equilibrium. Therefore, we have $d=\frac{1}{2}$, and player 2 claims $\left[0, \frac{1}{2}\right]$ exactly once. Next, we may assume without loss of generality that player 1 claims $\left[0, \frac{1}{10}\right]$ twice and $\left[\frac{1}{10}, 1\right]$ once: this is, essentially, the unique best reply. But then, player 2's marginal loss by removing a claim from $\left[0 ; \frac{1}{10}\right]$ would be $\frac{1}{3}$, while his marginal gain by adding a claim on $\left[\frac{1}{2}, 1\right]$ would be $\frac{1}{2}$. Hence, there is no Nash equilibrium.

In Proposition 4 we study claims problems where each player's claim contains an equal multiple of the estate. We show that in this class of claims problems the existence of a Nash equilibrium is guaranteed.

Proposition 4. Let $L \in \mathbb{N}$ and let $(E, \mathbf{c})$ be a claims problem with $c_{i}=L \cdot E+d_{i}$ and $0<d_{i} \leq E$ for every $i \in N$. Let $\left(\mathbf{x}, \alpha_{i}, m\right)_{i \in N}$ be a Nash equilibrium for restricted claims problem $(E, \mathbf{d})$. Then strategy profile $\left(\mathbf{x}, \beta_{i}, m\right)_{i \in N}$ with $\beta_{i}(t)=L+\alpha_{i}(t)$ for all $i \in N$ and $t \in M$ is a Nash equilibrium for $(E, \mathbf{c})$.

Proof. It is clear that Theorem 8 applies to Nash equilibrium $\left(\mathbf{x}, \alpha_{i}, m\right)_{i \in N}$ for restricted claims problem $(E, \mathbf{d})$. This gives rise to the following marginal loss and gain tables for strategy profile $\left(\mathbf{x}, \beta_{i}, m\right)_{i \in N}$, where - as in Theorem $8-K$ is the total claim on minimally claimed intervals in $\left(\mathbf{x}, \alpha_{i}, m\right)_{i \in N}$ and $K+1$ the total claim on maximally claimed intervals. In view of the characterization in Theorem 8 there are five possible cases to consider with respect to marginal losses and with respect to marginal gains which are given in Tables 5.3 and 5.4.

It can be verified that the marginal losses are ordered (iv) $>$ (v) $>$ (ii) $>$ (i) $>$ (iii) and the marginal gains are ordered (a) $>$ (d) $>$ (b) $>$ (e) $>$ (c). Clearly, it is sufficient to only check that the smallest marginal loss is at least as big as the largest 
Table 5.3: Marginal loss incurred by removing a claim from interval $t$.

\begin{tabular}{cccl} 
Case & $\beta_{N}(t)$ & $\beta_{i}(t)$ & marginal loss \\
\hline (i) & $n L+K+1$ & $L+1$ & $\frac{L+1}{n L+K+1}-\frac{L}{n L+K}$ \\
(ii) & $n L+K$ & $L+1$ & $\frac{L+1}{n L+K}-\frac{L}{n L+K-1}$ \\
(iii) & $n L+K$ & $L+2$ & $\frac{L+2}{n L+K}-\frac{L+1}{n L+K-1}$ \\
(iv) & $n L+K$ & $L$ & $\frac{L}{n L+K}-\frac{L-1}{n L+K-1}$ \\
(v) & $n L+K+1$ & $L$ & $\frac{L}{n L+K+1}-\frac{L-1}{n L+K}$ \\
\hline
\end{tabular}

Table 5.4: Marginal gain incurred by adding a claim to interval $t^{\prime}$.

\begin{tabular}{cccc}
\hline Case & $\beta_{N}\left(t^{\prime}\right)$ & $\beta_{i}\left(t^{\prime}\right)$ & marginal gain \\
\hline (a) & $n L+K$ & $L$ & $\frac{L+1}{n L+K+1}-\frac{L}{n L+K}$ \\
(b) & $n L+K$ & $L+1$ & $\frac{L+2}{n L+K+1}-\frac{L+1}{n L+K}$ \\
(c) & $n L+K$ & $L+2$ & $\frac{L+3}{n L+K+1}-\frac{L+2}{n L+K}$ \\
(d) & $n L+K+1$ & $L$ & $\frac{L+1}{n L+K+2}-\frac{L}{n L+K+1}$ \\
(e) & $n L+K+1$ & $L+1$ & $\frac{L+2}{n L+K+2}-\frac{L+1}{n L+K+1}$ \\
\hline
\end{tabular}

marginal gain. By Theorem 8 condition (A4) it follows that a shift of a claim from interval (iii) onto (a) and from (iv) onto (c) cannot occur. As a result we compare the marginal loss in case (i) with the marginal gain in case (a) and we compare the marginal loss in case (iii) with the marginal gain in case (d). From both tables it follows that the marginal loss in (i) is equal to the gain in (a). From (A4) it follows that a shift of one claim from (iii) to (d) can only occur in case $K \geq 4$ and thus $n \geq 5$. Using these facts it can be shown that the marginal loss in case (iii) is at least as big as the marginal gain in (d).

In a Nash equilibrium in Proposition 4, each player claims $L$ times the whole estate. The remainders of the claims are distributed over $[0, E]$ such as to form an equilibrium of the kind in Theorem 8.

The next example shows that there may be equilibria different from the ones described in Proposition 4.

Example 17. Consider claims problem $(E, \mathbf{c})$ with $E=4, n=4$ and $\mathbf{c}=(6,5,5,5)$. An equilibrium of the type of Proposition 4 is $\left(\mathbf{x}, \alpha_{i}, m\right)_{i \in N}$ with $m=4, \mathbf{x}=$ 
$(0,1,2,4), \alpha_{1}=(1,1,2), \alpha_{2}=(1,2,1), \alpha_{3}=\alpha_{4}=(2,1,1)$. Another equilibrium is $\left(\mathbf{x}, \alpha_{i}, m\right)_{i \in N}$ with $m=2, \mathbf{x}=(0,3,4), \alpha_{1}=(2,0), \alpha_{2}=\alpha_{3}=\alpha_{4}=(1,2)$. In this strategy profile, player 1 puts no claims on the interval $[3,4]$.

\subsubsection{Asymptotic result}

We conclude this section by showing that - not unexpectedly - the distributions resulting from Nash equilibria converge to the proportional distribution if the claims tend to infinity. ${ }^{6}$ In Lemmas $20-22$ and Theorem 9 we consider a sequence of claims problems $\left(E, \mathbf{c}^{k}\right)_{k=1}^{\infty}$ with $\mathbf{c}^{k}=\left(c_{1}^{k}, \ldots, c_{n}^{k}\right)$ for $k \in \mathbb{N}$. We assume that $\lim _{k \rightarrow \infty} c_{i}^{k}=\infty$ for all $i \in N$ and that $\mathbf{f}=\left(f_{1}, \ldots, f_{n}\right)$ exists such that $\lim _{k \rightarrow \infty} \mathbf{c}^{k} / \sum_{i \in N} c_{i}^{k}=\mathbf{f}$. If $f_{i}=1$ for some $i \in N$, then necessarily $f_{j}=0$ for $j \in N \backslash i$ and it is easy to see that player $i$ receives everything as $k \rightarrow \infty$, which is in accordance to the proportional distribution. Hence, we only consider the case $f_{i}<1$ for all $i \in N$.

We consider a sequence of strategy profiles $\left(\left(\mathbf{x}^{k}, \alpha_{i}^{k}, m^{k}\right)_{i \in N}\right)_{k=1}^{\infty}$ such that strategy profile $\left(\mathbf{x}^{k}, \alpha_{i}^{k}, m^{k}\right)_{i \in N}$ is a Nash equilibrium of $\left(E, \mathbf{c}^{k}\right)$ with payoff vector $y^{k}$ for each $k \in \mathbb{N}$ defined by (5.1). Let $M^{k}=\left\{1, \ldots, m^{k}\right\}$ for all $k \in \mathbb{N}$. For each $k \in \mathbb{N}$ we denote $\alpha_{N, \text { max }}^{k}=\max _{s^{k} \in M^{k}} \alpha_{N}^{k}\left(s^{k}\right)$ and $\alpha_{N, \min }^{k}=\min _{s^{k} \in M^{k}} \alpha_{N}^{k}\left(s^{k}\right)$ and $\alpha_{N \backslash i, \min }^{k}=\min _{s^{k} \in M^{k}} \alpha_{N \backslash i}^{k}\left(s^{k}\right)$ for all $i \in N$.

The next lemma shows that the minimum amount of claims on an interval converges to infinity as $k \rightarrow \infty$.

Lemma 20. $\lim _{k \rightarrow \infty} \alpha_{N, \min }^{k}=\infty$.

Proof. Obviously $\sum_{i \in N} c_{i}^{k} \leq \alpha_{N, \max }^{k} \cdot E$. Since $\lim _{k \rightarrow \infty} c_{i}^{k}=\infty$ for all $i \in N$, it follows that $\lim _{k \rightarrow \infty} \alpha_{N \text {,max }}^{k}=\infty$ and by Lemma 13 that $\lim _{k \rightarrow \infty} \alpha_{N, \text { min }}^{k}=\infty$.

Lemma 21. Let $\epsilon>0$. There exists an $s^{k} \in M^{k}$ for each $k \in \mathbb{N}$ such that for large $k$ and for all $i \in N$ it holds that $\frac{\alpha_{i}^{k}\left(s^{k}\right)}{\alpha_{N}^{k}\left(s^{k}\right)}<f_{i}+\epsilon$.

Proof. Let $\epsilon>0$. Take $i \in N$. Suppose, to the contrary, that for large $k$ we have

$$
\frac{\alpha_{i}^{k}\left(t^{k}\right)}{\alpha_{N}^{k}\left(t^{k}\right)}>f_{i}+\epsilon
$$

\footnotetext{
${ }^{6}$ A more direct proof is given in Atlamaz et al. [4] using one of the results in Atlamaz [3].
} 
for all $t^{k} \in M^{k}$. Hence, $\alpha_{i}^{k}\left(t^{k}\right)>\left(f_{i}+\epsilon\right) \alpha_{N}^{k}\left(t^{k}\right)$ for large $k$. Then, for large $k$,

$$
\begin{aligned}
\sum_{t^{k} \in M^{k}} \alpha_{i}^{k}\left(t^{k}\right)\left(x^{k}\left(t^{k}\right)-x^{k}\left(t^{k}-1\right)\right) & >\sum_{t^{k} \in M^{k}}\left(f_{i}+\epsilon\right) \alpha_{N}^{k}\left(t^{k}\right)\left(x^{k}\left(t^{k}\right)-x^{k}\left(t^{k}-1\right)\right) \\
& =\left(f_{i}+\epsilon\right) \sum_{t^{k} \in M^{k}} \alpha_{N}^{k}\left(t^{k}\right)\left(x^{k}\left(t^{k}\right)-x^{k}\left(t^{k}-1\right)\right) \\
& =\left(f_{i}+\epsilon\right) \sum_{j \in N} c_{j}^{k}>c_{i}^{k},
\end{aligned}
$$

which is a contradiction.

The next lemma shows that the number of claims of players $N \backslash i$ in any interval converges to infinity as $k \rightarrow \infty$.

Lemma 22. $\lim _{k \rightarrow \infty} \alpha_{N \backslash i, \min }^{k}=\infty$ for all $i \in N$.

Proof. Take $i \in N$. For each $k \in \mathbb{N}$ take $t^{k} \in M^{k}$. Suppose that $\left(\alpha_{N \backslash i}^{k}\left(t^{k}\right)\right)_{k=1}^{\infty}$ does not converge to infinity as $k \rightarrow \infty$. Then, without loss of generality, ${ }^{7}$ we can assume that $\alpha_{N \backslash i}^{k}\left(t^{k}\right)$ is bounded and thus, since $\alpha_{N}^{k}\left(t^{k}\right) \geq \alpha_{N, \text { min }}^{k}$ and by Lemma $20, \lim _{k \rightarrow \infty} \frac{\alpha_{N i k}^{k}\left(t^{k}\right)}{\alpha_{N}^{k}\left(t^{k}\right)}=0$. Since $f_{i}<1$ and by Lemma 21 , there exists an $\epsilon>0$ and for each $k \in \mathbb{N}$ an $s^{k} \in M$ such that $\frac{\alpha_{i}^{k}\left(s^{k}\right)}{\alpha_{N}^{k}\left(s^{k}\right)}<1-\epsilon<f_{i}$ for large $k$. Equivalently $\frac{\alpha_{N \backslash i}^{k}\left(s^{k}\right)}{\alpha_{N}^{k}\left(s^{k}\right)} \geq \epsilon$ for large $k$. Hence, for large $k$ it holds that

$$
\frac{\alpha_{N \backslash i}^{k}\left(s^{k}\right)}{\alpha_{N}^{k}\left(s^{k}\right)}>\frac{\alpha_{N \backslash i}^{k}\left(t^{k}\right)}{\alpha_{N}^{k}\left(t^{k}\right)} .
$$

(i) Suppose $\alpha_{N}^{k}\left(t^{k}\right)-\alpha_{N}^{k}\left(s^{k}\right)>2$. Then, for large $k$ we have

$$
\begin{aligned}
\frac{\alpha_{i}^{k}\left(s^{k}\right)+1}{\alpha_{N}^{k}\left(s^{k}\right)+1}-\frac{\alpha_{i}^{k}\left(s^{k}\right)}{\alpha_{N}^{k}\left(s^{k}\right)} & =\frac{\alpha_{N \backslash i}^{k}\left(s^{k}\right)}{\alpha_{N}^{k}\left(s^{k}\right)} \cdot \frac{1}{\alpha_{N}^{k}\left(s^{k}\right)+1} \\
& >\frac{\alpha_{N \backslash i}^{k}\left(t^{k}\right)}{\alpha_{N}^{k}\left(t^{k}\right)} \cdot \frac{1}{\alpha_{N}^{k}\left(t^{k}\right)-1} \\
& =\frac{\alpha_{i}^{k}\left(t^{k}\right)}{\alpha_{N}^{k}\left(t^{k}\right)}-\frac{\alpha_{i}^{k}\left(t^{k}\right)-1}{\alpha_{N}^{k}\left(t^{k}\right)-1}
\end{aligned}
$$

which contradicts condition (5.2) in Theorem 7.

\footnotetext{
${ }^{7}$ If a sequence of non-negative numbers does not diverge to infinity, then the sequence has a bounded subsequence.
} 
(ii) Suppose $\alpha_{N}^{k}\left(t^{k}\right)-\alpha_{N}^{k}\left(s^{k}\right) \leq 2$. Since $\frac{\alpha_{N \backslash\left(s^{k}\right)}^{k}}{\alpha_{N}^{k}\left(s^{k}\right)} \geq \epsilon$ for large $k$, we can choose $j(k) \neq i$ for each $k \in \mathbb{N}$, such that $\frac{\alpha_{j}^{k}\left(s^{k}\right)}{\alpha_{N}^{k}\left(s^{k}\right)} \geq \frac{\epsilon}{n-1}$ for large $k$. Moreover, since $\alpha_{N \backslash i}^{k}\left(t^{k}\right)$ is bounded, $\alpha_{j(k)}^{k}\left(t^{k}\right)$ is bounded and thus $\lim _{k \rightarrow \infty} \frac{\alpha_{j(k)}^{k}\left(t^{k}\right)}{\alpha_{N}^{k}\left(t^{k}\right)}=0$. Thus, for large $k$ it holds that $\frac{\alpha_{j(k)}^{k}\left(s^{k}\right)}{\alpha_{N}^{k}\left(s^{k}\right)}>\frac{\alpha_{j(k)}^{k}\left(t^{k}\right)}{\alpha_{N}^{k}\left(t^{k}\right)}$. In fact, $\frac{\alpha_{j(k)}^{k}\left(t^{k}\right)}{\alpha_{N}^{k}\left(t^{k}\right)} \leq \frac{\epsilon}{n}<\frac{\epsilon}{n-1}$ for large $k$, and thus for large $k$

$$
\frac{\alpha_{j(k)}^{k}\left(s^{k}\right)}{\alpha_{N}^{k}\left(s^{k}\right)}-\frac{\alpha_{j(k)}^{k}\left(t^{k}\right)}{\alpha_{N}^{k}\left(t^{k}\right)}>\frac{\epsilon}{n^{2}} .
$$

Because $\alpha_{N}^{k}\left(s^{k}\right) \geq \alpha_{N, \min }^{k}$ and $\alpha_{N}^{k}\left(t^{k}\right) \geq \alpha_{N, \text { min }}^{k}$ and by Lemma 20 it follows that $\lim _{k \rightarrow \infty} \frac{\alpha_{N}^{k}\left(s^{k}\right)+1}{\alpha_{N}^{k}\left(s^{k}\right)-1}=1$ and $\lim _{k \rightarrow \infty} \frac{\alpha_{N}^{k}\left(t^{k}\right)-1}{\alpha_{N}^{k}\left(t^{k}\right)+1}=1$. Then, for large $k$ we have

$$
\begin{aligned}
\frac{\alpha_{j(k)}^{k}\left(t^{k}\right)+1}{\alpha_{N}^{k}\left(t^{k}\right)+1}-\frac{\alpha_{j(k)}^{k}\left(t^{k}\right)}{\alpha_{N}^{k}\left(t^{k}\right)} & =\frac{\alpha_{N \backslash j(k)}^{k}\left(t^{k}\right)}{\alpha_{N}^{k}\left(t^{k}\right)} \cdot \frac{1}{\alpha_{N}^{k}\left(t^{k}\right)+1} \\
& =\left(1-\frac{\alpha_{j(k)}^{k}\left(t^{k}\right)}{\alpha_{N}^{k}\left(t^{k}\right)}\right) \cdot \frac{\alpha_{N}^{k}\left(t^{k}\right)-1}{\alpha_{N}^{k}\left(t^{k}\right)+1} \cdot \frac{1}{\alpha_{N}^{k}\left(t^{k}\right)-1} \\
& >\left(1-\frac{\alpha_{j(k)}^{k}\left(s^{k}\right)}{\alpha_{N}^{k}\left(s^{k}\right)}\right) \cdot \frac{\alpha_{N}^{k}\left(s^{k}\right)+1}{\alpha_{N}^{k}\left(s^{k}\right)-1} \cdot \frac{1}{\alpha_{N}^{k}\left(s^{k}\right)+1} \\
& =\frac{\alpha_{N \backslash j(k)}^{k}\left(s^{k}\right)}{\alpha_{N}^{k}\left(s^{k}\right)} \cdot \frac{1}{\alpha_{N}^{k}\left(s^{k}\right)-1} \\
& =\frac{\alpha_{j(k)}^{k}\left(s^{k}\right)}{\alpha_{N}^{k}\left(s^{k}\right)}-\frac{\alpha_{j(k)}^{k}\left(s^{k}\right)-1}{\alpha_{N}^{k}\left(s^{k}\right)-1},
\end{aligned}
$$

which contradicts condition (5.2) in Theorem 7.

Hence, $\alpha_{N \backslash i}^{k}\left(t^{k}\right)$ is not bounded. This is true for arbitrary $t^{k}$.

Lemmas 15 and 22 are used in Theorem 9 to show that the payoff $y^{k}$, as defined by (5.1), in Nash equilibrium converges to the proportional distribution.

Theorem 9. $\lim _{k \rightarrow \infty} y_{i}^{k}=f_{i} \cdot$ E for all $i \in N$.

Proof. Take $i \in N$. From Lemma 22 it follows that $\lim _{k \rightarrow \infty} \alpha_{N \backslash i \text {,min }}^{k}=\infty$. From Lemma 15 it follows that player $i$ 's payoff $y_{i}^{k}$ in the limit equals at least

$$
\lim _{k \rightarrow \infty}\left(\frac{c_{i}^{k}}{\sum_{j \in N} c_{j}^{k}}-\frac{1}{\alpha_{N \backslash i, \min }^{k}}\right) E=\lim _{k \rightarrow \infty} \frac{c_{i}^{k}}{\sum_{j \in N} c_{j}^{k}} E=f_{i} \cdot E .
$$


Since this holds for all $i \in N$, the distribution must be the proportional rule.

Theorem 9 can be interpreted in a different way which is shown in section 5.6.1.

\subsection{Variations on the basic model}

In the model considered so far, in a claims problem $(E, \mathbf{c})$ a player $i$ can put any nonnegative integer claim on some part of the estate, as long as total claims do not exceed $c_{i}$. We can express this by saying that the claim height is equal to 1 and players put multiples of this claim height on parts of the estate. What would happen if we change this claim height to some number $0<\gamma \leq 1$. Furthermore, what happens if we assume that players can choose from a finite or infinite number of claim heights, or even vary claim heights over each point of the interval $[0, E]$ for an estate $E$. In this section we study these variations.

\subsubsection{Variation 1: Fixed claim height smaller than 1}

Suppose the claim height is $\gamma$ with $0<\gamma \leq 1$. For a claims problem $(E, \mathbf{c})$ and a strategy profile $\left(\mathbf{x}, \alpha_{i}, m\right)_{i \in N}$ we interpret $\alpha_{i}(t)$ now as the number of claims of height $\gamma$ put on interval $t \in M$ by player $i \in N$. To distinguish such a strategy profile from a strategy profile with claim height 1 we will write $\left(\mathbf{x}, \alpha_{i}, m ; \gamma\right)_{i \in N}$. The associated payoff distribution is still given by (5.1). The feasibility constraint becomes $c_{i}=\sum_{t \in M} \alpha_{i}(t) \gamma(x(t)-x(t-1))$ for all $i \in N$. The obvious condition for a Nash equilibrium similar to (5.2) is

$$
\frac{\alpha_{i}(t) \gamma}{\alpha_{N}(t) \gamma}-\frac{\alpha_{i}(t) \gamma-\gamma}{\alpha_{N}(t) \gamma-\gamma} \geq \frac{\alpha_{i}\left(t^{\prime}\right) \gamma+\gamma}{\alpha_{N}\left(t^{\prime}\right) \gamma+\gamma}-\frac{\alpha_{i}\left(t^{\prime}\right) \gamma}{\alpha_{N}\left(t^{\prime}\right) \gamma}
$$

but this inequality is of course equivalent to (5.2). Together with the feasibility condition this implies that strategy profile $\left(\mathbf{x}, \alpha_{i}, m ; \gamma\right)_{i \in N}$ is a Nash equilibrium for $(E, \mathbf{c})$ if and only if strategy profile $\left(\mathbf{x}, \alpha_{i}, m\right)_{i \in N}=\left(\mathbf{x}, \alpha_{i}, m ; 1\right)_{i \in N}$ is a Nash equilibrium for $(E, \mathbf{c} / \gamma)$. A consequence of these considerations and Theorem 9 is that equilibrium distributions converge to the proportional distribution as the claim height goes to zero. Formally, we have the following result.

Corollary 1. Let $1 \geq \gamma^{1}>\gamma^{2}>\ldots$ with $\lim _{k \in \infty} \gamma^{k}=0$. Let $\left(\mathbf{x}^{k}, \alpha_{i}^{k}, m^{k} ; \gamma^{k}\right)_{i \in N}$ be a Nash equilibrium for $\left(E, \mathbf{c}^{k}\right)$ for each $k \in \mathbb{N}$ with payoff distribution $y^{k}$. Then $\lim _{k \rightarrow \infty} y^{k}=\mathbf{f} \cdot E$. 


\subsubsection{Variation 2: Arbitrary claim height}

For a claims problem $(E, \mathbf{c})$ and a strategy profile $\left(\mathbf{x}, \alpha_{i}, m\right)_{i \in N}$, we now assume that $\alpha_{i}(t) \in \mathbb{R}_{+}$for all $i \in N$ and all $t \in M$. We denote such a strategy profile by $\left(\mathbf{x}, \alpha_{i}, m ; \mathbb{R}_{+}\right)_{i \in N}$. The payoff distribution is given by (5.1) and the feasibility constraint is still $c_{i}=\sum_{t=1}^{m} \alpha_{i}(t)(x(t)-x(t-1))$ for each player $i$. The condition similar to (5.2) in Theorem 7 for a Nash equilibrium is now

$$
\frac{\alpha_{i}(t)}{\alpha_{N}(t)}-\frac{\alpha_{i}(t)-\epsilon}{\alpha_{N}(t)-\epsilon} \geq \frac{\alpha_{i}\left(t^{\prime}\right)+\epsilon}{\alpha_{N}\left(t^{\prime}\right)+\epsilon}-\frac{\alpha_{i}\left(t^{\prime}\right)}{\alpha_{N}\left(t^{\prime}\right)}
$$

for each $i \in N, t \in M$ with $\alpha_{i}(t)>0, t^{\prime} \in M$, and $0<\epsilon<\alpha_{i}(t)$. This inequality can be rewritten to

$$
\frac{\alpha_{N}(t)-\alpha_{i}(t)}{\alpha_{N}(t)\left(\alpha_{N}(t)-\epsilon\right)} \geq \frac{\alpha_{N}\left(t^{\prime}\right)-\alpha_{i}\left(t^{\prime}\right)}{\alpha_{N}\left(t^{\prime}\right)\left(\alpha_{N}\left(t^{\prime}\right)+\epsilon\right)}
$$

and this is satisfied for each $\epsilon>0$ if and only if

$$
\frac{\alpha_{N \backslash i}(t)}{\left(\alpha_{N}(t)\right)^{2}} \geq \frac{\alpha_{N \backslash i}\left(t^{\prime}\right)}{\left(\alpha_{N}\left(t^{\prime}\right)\right)^{2}}
$$

Note that, if $\alpha_{i}\left(t^{\prime}\right)>0$ as well, then also the converse of this inequality has to hold, hence we have equality.

Consider any strategy profile $\left(\mathbf{x}, \alpha_{i}, m ; \mathbb{R}_{+}\right)_{i \in N}$ and a player $i \in N$. Given the strategy profile restricted to $N \backslash i$, namely $\left(\mathbf{x}, \alpha_{j}, m ; \mathbb{R}_{+}\right)_{j \in N \backslash i}$, and assuming ${ }^{8}$ that $\alpha_{N \backslash i}(t)>0$ for all $t \in M$, player $i$ can guarantee a payoff of $\left(c_{i} / \sum_{j \in N} c_{j}\right) E$ by claiming according to $\alpha_{i}^{\prime}$ defined by

$$
\alpha_{i}^{\prime}(t)=\frac{c_{i}}{\sum_{j \in N \backslash i} c_{j}} \alpha_{N \backslash i}(t)
$$

for all $t \in M$. This way, player $i$ obtains his proportional share of every inter$\mathrm{val}$, and thus of the estate $E$. Since this is true for every player $i$, every Nash equilibrium for $(E, \mathbf{c})$ must result in the proportional distribution. Moreover, it follows easily from (5.5) that $\left(\mathbf{x}, \alpha_{i}, 1 ; \mathbb{R}_{+}\right)_{i \in N}-$ thus, each player $i$ putting a claim of height $c_{i} / E$ on the whole interval $[0, E]-$ is a Nash equilibrium. We call this profile the uniform strategy profile. In fact, the uniform strategy profile

\footnotetext{
${ }^{8}$ If this assumption is not satisfied then player $i$ can even do better by putting a claim of very small height on the non-claimed part(s) of the interval $[0, E]$.
} 
is the unique ${ }^{9}$ Nash equilibrium among the strategy profiles $\left(\mathbf{x}, \alpha_{i}, m ; \mathbb{R}_{+}\right)_{i \in N}$. This can be seen as follows. Let $\left(\mathbf{x}, \alpha_{i}, m ; \mathbb{R}_{+}\right)_{i \in N}$ be a Nash equilibrium different from the uniform strategy profile. Clearly, we may assume $\alpha_{N}(t)>0$ for all $t \in M$. Now there must be an $i \in N$ and $t, t^{\prime} \in M$ such that $\alpha_{N \backslash i}(t)>$ $\sum_{j \in N \backslash i} c_{j} / E>\alpha_{N \backslash i}\left(t^{\prime}\right)$. This is so since against a total claim of $N \backslash i$, player $i$ 's unique best reply is to claim uniformly - this follows from (5.5). By claiming proportionally according to $\alpha_{i}^{\prime}$ as above, player $i$ obtains his proportional payoff $\left(c_{i} / \sum_{j \in N} c_{j}\right) E$. In that case, we have

$$
\frac{\alpha_{N \backslash i}(s)}{\left(\alpha_{N}(s)\right)^{2}}=\frac{1}{\alpha_{N \backslash i}(s)\left(1+c_{i} / \sum_{j \in N} c_{j}\right)^{2}}
$$

for $s=t, t^{\prime}$. Therefore, (5.5) is violated, implying that player $i$ can improve his payoff by transferring part of his claim from $t$ to $t^{\prime}$. This, in turn, implies that his equilibrium payoff is higher than proportional, a contradiction.

Summarizing, we have the following result.

Proposition 5. Among all strategy profiles $\left(\mathbf{x}, \alpha_{i}, m ; \mathbb{R}_{+}\right)_{i \in N}$ for claims problem $(E, \mathbf{c})$, the uniform strategy profile, resulting in the proportional payoff distribution $E c / \sum_{i \in N} c_{i}$, is the unique Nash equilibrium.

\subsubsection{Variation 3: Restricted arbitrary claim height}

A variation in-between variation 1 and variation 2 is the following. For a claims problem $(E, \mathbf{c})$, player $i$ first divides his claim, the rectangle with height 1 and width $c_{i}$, into smaller rectangles by choosing a finite number of cuts on the horizontal interval $\left[0, c_{i}\right]$ and a finite number of cuts on the vertical interval $[0,1]$. Next, a player puts these rectangular claims on the estate $[0, E]$. The placement of rectangular claims is restricted; it cannot be turned.

It can be shown that still any Nash equilibrium must result in the proportional distribution. However, the uniform strategy profile may not be feasible. It is the 'coarsest' division of claims for which it still holds that players receive the proportional outcome in equilibrium.

\footnotetext{
${ }^{9}$ In the sense of numbers of claims on each part of the estate.
} 


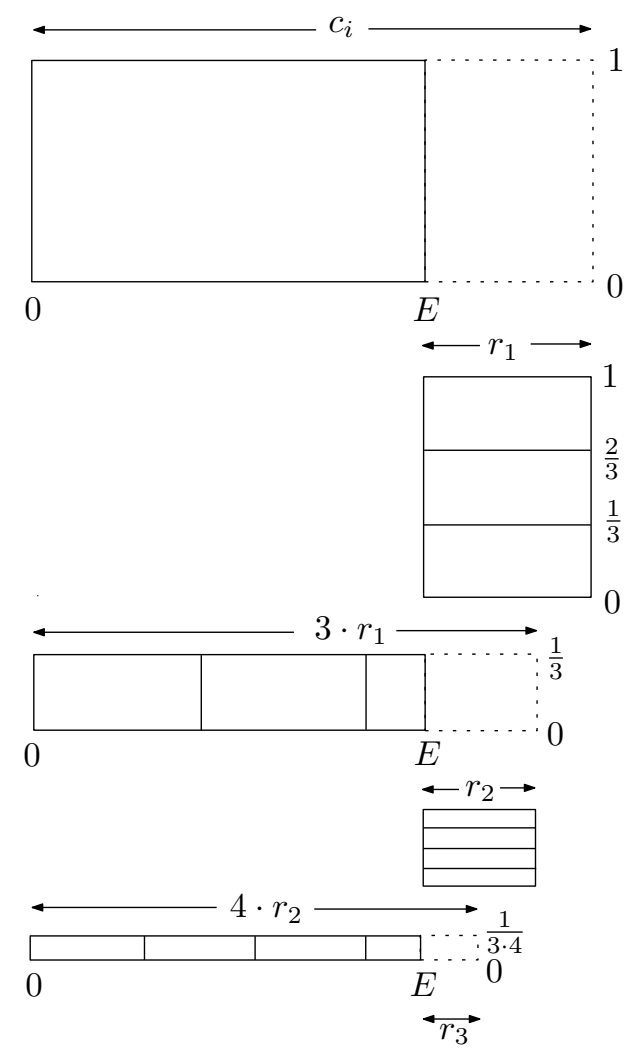

Figure 5.1: Specific claim division in model variation 3. Example of a recursive system with $L=1, k_{1}=3$ and $k_{2}=4$, etc. 
We first specify a construction which defines how a claim is placed on the estate.

Consider a claim of player $i$ with $L \cdot E \leq c_{i}<(L+1) E$ for $L \in \mathbb{N}_{0}$. Let player $i$ place the amount $L \cdot E$ uniformly on the estate. Define the remainder $r_{1} \equiv$ $c_{i}-L \cdot E$. If $r_{1}=0$, then the whole claim is placed and we are done. If $r_{1} \neq 0$, then there is a $k_{1} \in \mathbb{N}$ such that $\left(k_{1}-1\right) r_{1}<E \leq k_{1} \cdot r_{1}$. Player $i$ then divides the height of remainder $r_{1}$ which is equal to 1 in $k_{1}$ equal parts. As a result player $i$ has $k_{1}$ times part $r_{1}$ with height $1 / k_{1}$. Clearly, it is possible for player $i$ to fit part $\left(k_{1}-1\right) r_{1}$ with height $1 / k_{1}$ on interval $[0, E]$, however $k_{1} \cdot r_{1}$ with the same height does not completely fit to the interval; a part of size $r_{2}=k_{1} \cdot r_{1}-E$ with height $1 / k_{1}$ exceeds $[0, E]$. Of course if $r_{2}=0$, then the whole claim $c_{1}$ is placed and we are done, otherwise there is a $k_{2}$ such that $\left(k_{2}-1\right) r_{2} \leq E<k_{2} \cdot r_{2}$ and player $i$ divides the height of the remainder $r_{2}$ which is $1 / k_{1}$ in $k_{2}$ equal parts. Then he places $k_{2} \cdot r_{2}$ with height $1 /\left(k_{1} \cdot k_{2}\right)$ on $[0, E]$, which gives a remainder of $r_{3}=k_{2} \cdot r_{2}-E$. If $r_{3}=0$ then we are done, otherwise we apply the same trick as before. Etc. An example is given in Figure 5.1.

Define $r_{1} \equiv c_{i}-L \cdot E$. Let $t \in \mathbb{N}$ be fixed and suppose $r_{t}$ is known then ${ }^{10}$

$$
\begin{aligned}
k_{t} & =\left\lceil\frac{E}{r_{t}}\right\rceil \\
r_{t+1} & =k_{t} \cdot r_{t}-E .
\end{aligned}
$$

Some interesting results are derived in the next lemma about the defined recursive system.

Lemma 23. Consider a recursive system defined by both parts in equation (5.6) with $E, c_{i} \in \mathbb{N}$.

(i) Then $\lim _{t \rightarrow \infty} r_{t}=0$.

(ii) Moreover, if $\frac{c_{i}}{E} \in \mathbb{Q}$, then there exists a $t \in \mathbb{N}$ such that $r_{t}=0$.

Proof. Let $\epsilon>0$. (i) From both parts in (5.6) it follows that $r_{2}=k_{1} \cdot r_{1}-E<r_{1}$. We can extend this result and find that $r_{t+1}<r_{t}$ for all $t \in \mathbb{N}$, which means that $r_{t}$ converges. Suppose, to the contrary, that $\lim _{t \rightarrow \infty} r_{t}=a>0$. There exists a large $T \in \mathbb{N}$ with $r_{T}=a+\frac{\epsilon}{k}$ where $k=\left\lceil\frac{E}{a}\right\rceil \in \mathbb{N}$. Define $a^{\prime}=k \cdot a-E<a$. Next, there exists a unique $k_{T} \in \mathbb{N}$ with $k_{T}=\left\lceil\frac{E}{r_{T}}\right\rceil$. From (5.6b) it follows that

$$
r_{T+1}=k_{T} \cdot r_{T}-E \leq k\left(a+\frac{\epsilon}{k}\right)-E=a^{\prime}+\epsilon
$$

\footnotetext{
${ }^{10} \mathrm{By}\lceil x\rceil$ we denote the ceil of $x$ and is defined as follows $\lceil x\rceil:=\min \{z \in \mathbb{Z} \mid z \geq x\}$.
} 
The inequality follows because $k_{T} \leq k$. In order to see this consider $k=\left\lceil\frac{E}{a}\right\rceil$ and $k_{T}=\left\lceil\frac{E}{r_{T}}\right\rceil=\left\lceil\frac{E}{a+\delta}\right\rceil$. Clearly, since $r_{T}>a$ we have $\delta>0$ and as a result $\frac{E}{a+\delta}<\frac{E}{a}$, from which it follows that $k_{T} \leq k$. From (5.7) we can conclude that $r_{T+1}$ is close to $a^{\prime}<a$. Since this is true for arbitrary $a$, we have shown that $\lim _{t \rightarrow \infty} r_{t}=0$.

(ii) Let $\frac{c_{i}}{E} \in \mathbb{Q}$. From the definition of $r_{1}$ it follows that $\frac{r_{1}}{E}=\frac{c_{i}}{E}-L \in \mathbb{Q}$. Equation (5.6b) shows that $\frac{r_{t+1}}{E}=k_{t} \frac{r_{t}}{E}-1$ and because $\frac{r_{1}}{E} \in \mathbb{Q}$ it follows that $\frac{r_{t+1}}{E} \in \mathbb{Q}$ for all $t$. As a result $\frac{r_{t}}{E} \in \mathbb{Q}$ for all $t$. Thus we can write $\frac{r_{1}}{E}=\frac{m}{n}$ for $m, n \in \mathbb{Z}$ such that $\frac{m}{n} \in \mathbb{R}_{+}$. Because $r_{1}=c_{i}-L E<E$ we have $m<n$. By (5.6a) we obtain $k_{1}=\left\lceil\frac{n}{m}\right\rceil$ for $t=1$, which simply means that $k_{1}-1<\frac{n}{m} \leq k_{1}$ for $k_{1} \in \mathbb{N}$. By both parts in (5.6) it follows that $\frac{r_{2}}{E}=k_{1} \frac{m}{n}-1=\frac{k_{1} \cdot m-n}{n}<\frac{m}{n}=\frac{r_{1}}{E}$ for $t=2$. The inequality follows from the fact that $k_{1}-1<\frac{n}{m}$ and thus $k_{1} \cdot m-n<m$. Similarly we find $\frac{r_{t+1}}{E}<\frac{r_{t}}{E}$, hence $r_{t}$ converges. Because $\frac{r_{t}}{E}$ is of the form $\frac{k_{t-1} \cdot m-n}{n}$, it follows that there is a finite $t$ for which $r_{t}=0$.

Corollary 2 consists of two parts. The first part immediately follows from Lemma 23(ii) and shows that under special conditions on a player's claim and the estate, a player has a uniform strategy, i.e. a strategy where he puts a claim of height $c_{i}$ on the whole interval $[0, E]$. In the second part it is shown that the uniform strategy profile, is the unique Nash equilibrium.

\section{Corollary 2.}

(i) If $\frac{c_{i}}{E} \in \mathbb{Q}$ for $i \in N$, then a strategy exists where player $i$ divides his claim uniformly over the estate.

(ii) The strategy profile where all players have the strategy as described in (i) is the unique Nash equilibrium.

Proof. (i) Let $\frac{c_{i}}{E} \in \mathbb{Q}$ for $i \in N$. From Lemma 23(ii) it follows that there exists a $t \in \mathbb{N}$ such that $r_{t}=0$. Hence, the whole claim has been placed according to the recursive system described by both parts in equation (5.6), Which means that $c_{i}$ is uniformly divided over the estate.

(ii) Let $\frac{c_{i}}{E} \in \mathbb{Q}$ for all $i \in N$, it follows from (i) that all players then have a strategy where their claim is divided uniformly over the estate. From Proposition 5 it follows that this uniform strategy profile is the unique Nash equilibrium.

Next, we show that in variation 3 an individual can always play a strategy such that he receives a payoff which is at least equal to the proportional division minus an error term, independent of other players' strategies. 
Lemma 24. Let $\epsilon>0$. Given an arbitrary strategy profile of players $N \backslash i$, player $i$ can play a strategy which generates him a payoff of at least $E c_{i} / \sum_{j \in N} c_{j}-\epsilon$.

Proof. Let players $N \backslash i$ have an arbitrary strategy profile such that $[0, E]$ is divided in $m$ intervals by division $\mathbf{x}$. Let $M=\{1, \ldots, m\}$ and for all $l \in M$ let $h(l) \geq 0$ denote the total number of claims put on interval $[x(l-1), x(l)]$ by players $N \backslash i$. Furthermore, $\sum_{l \in M} h(l)(x(l)-x(l-1))=\sum_{j \in N \backslash i} c_{j}$. Take $s_{i}=c_{i} / \sum_{j \in N \backslash i} c_{j}$. If player $i$ puts $s_{i} \cdot h(l)$ claims on interval $[x(l-1), x(l)]$ for all $l \in M$, then he receives a part of the estate proportional to his claim. Moreover, he places a claim on interval $[x(l-1), x(l)]$ with surface $O(l):=s_{i} h(l)(x(l)-x(l-1))$ for every $l \in M$ and as can be verified $\sum_{l \in M} O(l)=c_{i}$.

Let $\delta>0$ and let

$$
\epsilon=\delta \frac{\sum_{j \in N \backslash i} c_{j}}{\sum_{j \in N} c_{j}} \sum_{l \in M} \frac{x(l)-x(l-1)}{h(l)} .
$$

Claiming $s_{i} \cdot h(l)-\delta$ on interval $[x(l-1), x(l)]$ for every $l \in M$ yields player $i$ payoff

$$
\begin{aligned}
\sum_{l \in M} & \frac{s_{i} \cdot h(l)-\delta}{\left(1+s_{i}\right) h(l)-\delta}(x(l)-x(l-1)) \\
& \geq \sum_{l \in M} \frac{s_{i} \cdot h(l)-\delta}{\left(1+s_{i}\right) h(l)}(x(l)-x(l-1)) \\
& =\sum_{l \in M} \frac{s_{i} h(l)}{\left(1+s_{i}\right) h(l)}(x(l)-x(l-1))-\sum_{l \in M} \frac{\delta}{\left(1+s_{i}\right) h(l)}(x(l)-x(l-1)) \\
& =\frac{c_{i}}{\sum_{j \in N} c_{j}} E-\epsilon
\end{aligned}
$$

Next, we define $P(l)=O(l) / c_{i}$ for all $l \in M$ and place $P(l) \cdot c_{i}$ according to the recursive system defined by both parts in (5.6) on interval $[x(l-1), x(l)]$ for all $l \in M$. This means that for every $l \in M$ there is an $L(l) \in \mathbb{N}_{0}$ such that $L(l)(x(l)-$ $x(l-1)) \leq c_{i}<(L(l)+1)(x(l)-x(l-1))$. For all $l \in M$ define $r_{1}(l) \equiv c_{i}-L(l)(x(l)-$ $x(l-1))$. Let $t \in \mathbb{N}$ be fixed and suppose $r_{t}(l)$ is known for all $l \in M$, then

$$
\begin{aligned}
k_{t}(l) & =\left\lceil\frac{x(l)-x(l-1)}{r_{t}(l)}\right\rceil \\
r_{t+1}(l) & =k_{t}(l) r_{t}(l)-(x(l)-x(l-1)) .
\end{aligned}
$$

Let $k_{0}(l)=1$ for all $l \in M$. From Lemma 23(i) it follows that $\lim _{t \rightarrow \infty} r_{t}(l)=0$ for all $l \in M$. As a result there exists a $t \in \mathbb{N}$ such that for all $l \in M$ we have

$$
r_{t}(l) \cdot P(l) \cdot \prod_{\nu=0}^{t-1} \frac{1}{k_{v}(l)}<\delta(x(l)-x(l-1))
$$


From this inequality it immediately follows that the amount claimed by player $i$ using the recursive system, which equals $O(l)-r_{t}(l) \cdot P(l) \cdot \prod_{v=0}^{t-1} \frac{1}{k_{v}(l)}$, is bigger than the amount necessary to obtain a payoff equal to $E c_{i} / \sum_{i \in N} c_{i}-\epsilon$. Player $i$ can play a strategy which generates him at least $E c_{i} / \sum_{i \in N} c_{i}-\epsilon$.

\subsubsection{Variation 4: Arbitrary claim height point-wise}

Unlike the model variations described before, this variation deals with an infinite number of intervals. We assume that a claims profile $(E, \mathbf{c})$ is any $n$-tuple of continuous functions $\alpha_{i}:[0, E] \rightarrow \mathbb{R}_{+}$such that $\int_{0}^{E} \alpha_{i}(t) d t=c_{i}$ for each $i \in N$. Denote $\alpha_{N}(t)=\sum_{i \in N} \alpha_{i}(t)$ for each $t \in[0, E]$, and, for each $i \in N$ and $t \in[0, E]$,

$$
\hat{\alpha}_{i}(t)= \begin{cases}\frac{\alpha_{i}(t)}{\alpha_{N}(t)} & \text { if } \alpha_{N}(t)>0, \\ 0 & \text { if } \alpha_{N}(t)=0 .\end{cases}
$$

Then the payoff distribution associated with strategy profile $\alpha=\left(\alpha_{1}, \ldots, \alpha_{n}\right)$ is for every $i \in N$ given by

$$
y_{i}=\int_{0}^{E} \hat{\alpha}_{i}(t) d t .
$$

Within this model the uniform strategy profile is the profile $u=\left(u_{1}, \ldots, u_{n}\right)$ defined by $u_{i}(t)=c_{i} / E$ for all $i \in N$ and $t \in[0, E]$. In this case, by arguments analogous to those in variation 2 we obtain the following result.

Proposition 6. Among all strategy profiles $\alpha=\left(\alpha_{1}, \ldots, \alpha_{n}\right)$ for claims problem $(E, \mathbf{c})$, the uniform strategy profile $u$, resulting in the proportional payoff distribution $E c / \sum_{i \in N} c_{i}$, is the unique Nash equilibrium.

\subsection{Conclusions and further research}

Extending an approach initiated by O'Neill [29], Chapter 5 offers non-cooperative solutions to claims problems. We observed in Example 14 that the proportional rule does not always pick equilibrium payoff distributions. ${ }^{11}$ This observation raises the question if there is an axiomatic, normative justification for the 'rule' found in this chapter, namely the correspondence that assigns the set of Nash equilibrium payoff distributions to each claims problem.

\footnotetext{
${ }^{11}$ In proposition 7 of Atlamaz [3] this is shown also for other well known bankruptcy rules.
} 
We defined our payoff such that every player receives from every point on the estate an amount proportional to his number of claims on that point. Therefore, it is not surprising that the payoff in Nash equilibrium tends towards the proportional rule. This raises the issue of what happens if another principle is used to define the payoffs in this game. For example, does the non-cooperative solution tend towards the weighted proportional rule if the weighted proportional rule is used on every point on the estate? To already elaborate a bit more on this topic, consider the constrained equal losses rule, which is defined as $C E L_{i}=\max \left\{c_{i}-\lambda, 0\right\}$ where $\lambda$ is such that $\sum_{i \in N} C E L_{i}=E$. If the division rule on every point on the estate is the constrained equal losses rule, then no Nash equilibrium exists if the sum of claims exceeds the estate. This follows from the fact that on every interval a player wins the interval simply if he has most claims on it. The interval is divided equally among players who have the same maximal claim on it. If a player does not have most claims, he can improve by moving his claim to another part, which can be done by all players. ${ }^{12}$

Furthermore, research could drop the implicit assumption of a homogeneous estate and consider claims problems where players preferences over the estate are not homogenous. Dropping this assumption leads to a model closely related to models of land division or, more generally, division of heterogeneous goods, see among others Berliant [9], Berliant and Ten Raa [10], Berliant et al. [11] and Legut et al. [26].

\subsection{Appendix}

Proof of Theorem 7. (i) Let strategy profile $\left(\mathbf{x}, \alpha_{i}, m\right)_{i \in N}$ be a Nash equilibrium. We have to show that in Nash equilibrium (A) $\alpha_{N}(t) \geq 1$ for all $t \in M$ and (B) condition (5.2) holds for all players.

(A) Assume, contrary to what we want to show, that there exists a $t \in M$ with $\alpha_{N}(t)=0$. Since $\sum_{i \in N} c_{i} \geq E$, there exists a $t^{\prime} \in M$ such that $\alpha_{N}\left(t^{\prime}\right) \geq 2$. Player $i$ 's marginal gain incurred from putting one claim on interval $t$ equals 1 , whereas his marginal loss incurred from claiming interval $t^{\prime}$ one time less is always less than 1 since $\alpha_{N}\left(t^{\prime}\right) \geq 2$. Hence, player $i$ can improve, which contradicts that $\left(\mathbf{x}, \alpha_{i}, m\right)_{i \in N}$ is a Nash equilibrium. Thus, $\alpha_{N}(t) \geq 1$ for all $t \in M$.

(B) Assume, to the contrary, that there is a player $i \in N$ for which there exist

\footnotetext{
${ }^{12}$ This different way of sharing is also mentioned in Atlamaz [3] Ch. 5.2.
} 
$t, t^{\prime} \in\{1, \ldots, m\}$ such that for $\alpha_{i}(t) \geq 1$ and $\alpha_{N}(t)>1$ it holds that

$$
\frac{\alpha_{i}(t)}{\alpha_{N}(t)}-\frac{\alpha_{i}(t)-1}{\alpha_{N}(t)-1}<\frac{\alpha_{i}\left(t^{\prime}\right)+1}{\alpha_{N}\left(t^{\prime}\right)+1}-\frac{\alpha_{i}\left(t^{\prime}\right)}{\alpha_{N}\left(t^{\prime}\right)}
$$

Inequality (5.9) says that the loss which player $i$ occurs by taking away a claim (or part of a claim) from some interval $t$ is smaller than his gain obtained from placing that claim on some other interval $t^{\prime}$. This means that player $i$ can improve, which contradicts that $\left(\mathbf{x}, \alpha_{i}, m\right)_{i \in N}$ is a Nash equilibrium.

(ii) Assume strategy profile $\left(\mathbf{x}, \alpha_{i}, m\right)_{i \in N}$ is no Nash equilibrium. Suppose player $i$ 's strategy $\left(\mathbf{x}, \alpha_{i}, m\right)$ is not a best response against $\left(\mathbf{x}, \alpha_{j}, m\right)_{j \in N \backslash i}$. Suppose player $i$ 's strategy $\left(\overline{\mathbf{x}}_{i}, \tilde{\alpha}_{i}, m_{i}\right)$ is a best response against $\left(\mathbf{x}, \alpha_{j}, m\right)_{j \in N \backslash i}$. Let $\overline{\mathbf{x}}$ with $\bar{m}$ intervals be the common refinement of players' partitions $\mathbf{x}$ and $\overline{\mathbf{x}}_{i}$ and let $\left(\bar{\alpha}_{i}\right)_{i \in N}$ be the according claims vector. Hence, strategy profile $\left(\left(\overline{\mathbf{x}}_{i}, \tilde{\alpha}_{i}, m_{i}\right)\right.$, $\left.\left(\mathbf{x}, \alpha_{j}, m\right)_{j \in N \backslash i}\right)$ is equivalent to strategy profile $\left(\overline{\mathbf{x}}, \bar{\alpha}_{i}, \bar{m}\right)_{i \in N}$.

To be able to compare strategy profiles $\left(\mathbf{x}, \alpha_{i}, m\right)_{i \in N}$ and $\left(\overline{\mathbf{x}}, \bar{\alpha}_{i}, \bar{m}\right)_{i \in N}$, we introduce $m^{\prime} \in \mathbb{N}_{0}$ with $m^{\prime} \geq \max \{m, \bar{m}\}, \mathbf{z} \in \mathbb{R}^{m^{\prime}+1}$ and $\beta, \bar{\beta} \in \mathbb{N}_{0}^{\left(N \times m^{\prime}\right)}$ with

$$
\beta_{i}(t)=\left\{\alpha_{i}(l) \mid[z(t-1), z(t)] \subseteq[x(l-1), x(l)], 1 \leq l \leq m\right\}
$$

and

$$
\bar{\beta}_{i}(t)=\left\{\bar{\alpha}_{i}(l) \mid[z(t-1), z(t)] \subseteq[\bar{x}(l-1), \bar{x}(l)], 1 \leq l \leq \bar{m}\right\}
$$

for every $t \in\left\{1, \ldots, m^{\prime}\right\}$ and every $i \in N$. Vector $\mathbf{z}$ contains all elements in $\overline{\mathbf{x}}$. Vector $\overline{\mathbf{x}}$ divides claim $c_{i}$ from left to right in little intervals. When $c_{i}$ is placed according to strategy profile $\left(\mathbf{x}, \alpha_{i}, m\right)_{i \in N}$ from left to right the division of $c_{i}$ caused by $\overline{\mathbf{x}}$ leaves extra partition points which are contained in vector $\mathbf{z}$. For all elements in $\mathbf{z}$ it holds that $0=z(0)<z(1)<\ldots<z\left(m^{\prime}\right)=E$. Partition $\mathbf{z}$ ensures that strategy profile $\left(\overline{\mathbf{x}}, \bar{\alpha}_{i}, \bar{m}\right)_{i \in N}$ can be obtained from strategy profile $\left(\mathbf{x}, \alpha_{i}, m\right)_{i \in N}$ by changing claims on equal length intervals in $\mathbf{z}$. Hence, we can compare strategy profile $\left(\mathbf{z}, \beta_{i}, m^{\prime}\right)_{i \in N}$ to strategy profile $\left(\mathbf{z}, \bar{\beta}_{i}, m^{\prime}\right)_{i \in N}$.

We choose the 'shortest path' to go from strategy profile $\left(\mathbf{z}, \beta_{i}, m^{\prime}\right)_{i \in N}$ to strategy profile $\left(\mathbf{z}, \bar{\beta}_{i}, m^{\prime}\right)_{i \in N}$. This means that we look at the minimum amount of elementary changes, starting with the elementary change of $\beta_{i}$, which are necessary to obtain $\bar{\beta}_{i}$. This means that on an interval $t$ with $\beta_{i}(t)>\bar{\beta}_{i}(t)$ only claims have been taken away one by one in order to obtain best response claim $\bar{\beta}_{i}(t)$ from $\beta_{i}(t)$. There has been no shift of claims involving $t$ where a claim 
has been added. If $p \geq 1$ elementary changes are applied which all change the number of claims on interval $t$ in order to obtain $\bar{\beta}_{i}(t)$ from $\beta_{i}(t)$, then starting with an elementary change of $\beta_{i}$, it follows that $\bar{\beta}_{i}(t)=\beta_{i}(t)-p$. Similarly, for an interval $t$ where $\beta_{i}(t)<\bar{\beta}_{i}(t)$ only claims have been put on $t$ one by one in order to obtain best response claim $\bar{\beta}_{i}(t)$ from $\beta_{i}(t)$. Hence, if player $i$ starts with $\beta_{i}(t)$ claims on interval $t$ and has to apply $q \geq 1$ elementary changes which all change the number of claims on interval $t$ in order to obtain $\bar{\beta}_{i}(t)$, then $\bar{\beta}_{i}(t)=\beta_{i}(t)+q$. In case $\beta_{i}(t)=\bar{\beta}_{i}(t)$ on an interval $t$, then interval $t$ was never involved in a reshuffling of claims, which means that no elementary changes have been applied.

By design, player $i$ 's payoff of strategy profile $\left(\mathbf{z}, \beta_{i}, m^{\prime}\right)_{i \in N}$ is lower than his payoff resulting from strategy profile $\left(\mathbf{z}, \bar{\beta}_{i}, m^{\prime}\right)_{i \in N}$, as can be verified by (5.1). Furthermore, to obtain strategy profile $\left(\mathbf{z}, \bar{\beta}_{i}, m^{\prime}\right)_{i \in N}$ from strategy profile $\left(\mathbf{z}, \beta_{i}, m^{\prime}\right)_{i \in N}$ a minimum amount of elementary changes are applied by player $i$, starting with an elementary change of $\beta_{i}$ and ending with an elementary change that leads immediately to player $i$ 's best response strategy $\left(\mathbf{z}, \bar{\beta}_{i}, m^{\prime}\right)$ given $\left(\mathbf{z}, \beta_{j}, m^{\prime}\right)_{j \in N \backslash i}$ of players $N \backslash i$. This last elementary change either (A) is an improvement for player $i$ or (B) it does not affect player $i$ 's payoff at all. Let $s, s^{\prime} \in\left\{1, \ldots, m^{\prime}\right\}$.

(A) If the final elementary change is an improvement for player $i$, then

$$
\frac{\beta_{i}(s)-p}{\beta_{N}(s)-p}-\frac{\beta_{i}(s)-p-1}{\beta_{N}(s)-p-1}<\frac{\beta_{i}\left(s^{\prime}\right)+q+1}{\beta_{N}\left(s^{\prime}\right)+q+1}-\frac{\beta_{i}\left(s^{\prime}\right)+q}{\beta_{N}\left(s^{\prime}\right)+q}
$$

for $s, s^{\prime} \in\left\{1, \ldots, m^{\prime}\right\}$ and some $p, q \geq 0$. Inequality (5.11) says that the loss which player $i$ occurs by taking away a claim from interval $s$ and as a result claiming it $\beta_{i}(s)-p-1=\bar{\beta}_{i}(s)$ times, is smaller than his gain obtained from placing that claim on interval $s^{\prime}$ and as a result claiming it $\beta_{i}\left(s^{\prime}\right)+q+1=\bar{\beta}_{i}\left(s^{\prime}\right)$ times.

(B) In case player $i$ 's payoff remains the same, we do not implement this elementary change and look at the penultimate elementary change. This elementary change is again either an improvement (case (A)) or player $i$ 's payoff is not affected by it (case (B)). Since player $i$ 's payoff of $\left(\mathbf{z}, \beta_{i}, m^{\prime}\right)_{i \in N}$ is lower than his payoff of $\left(\mathbf{z}, \bar{\beta}_{i}, m^{\prime}\right)_{i \in N}$, there is eventually an improvement as described in case (A), this does not lead to player $i$ 's best response strategy $\left(\overline{\mathbf{x}}_{i}, \bar{\alpha}_{i}, m_{i}\right)$. However, the resulting strategy yields player $i$ the same payoff as his best response strategy.

From case (A) and (B) it follows that player $i$ 's final implemented elementary change in order to reach his best response strategy or a strategy that yields 
the same payoff as his best response strategy, is always an improvement for him.

Suppose the final elementary change on intervals $s$ and $s^{\prime}$ is applied first, thereby it becomes the first elementary change of $\beta_{i}$. By (5.11) and decreasing marginal utility of player $i$ 's payoff function, we have for $p, q \geq 0$ that

$$
\begin{aligned}
\frac{\beta_{i}(s)}{\beta_{N}(s)}-\frac{\beta_{i}(s)-1}{\beta_{N}(s)-1} & \leq \frac{\beta_{i}(s)-p}{\beta_{N}(s)-p}-\frac{\beta_{i}(s)-p-1}{\beta_{N}(s)-p-1} \\
& <\frac{\beta_{i}\left(s^{\prime}\right)+q+1}{\beta_{N}\left(s^{\prime}\right)+q+1}-\frac{\beta_{i}\left(s^{\prime}\right)+q}{\beta_{N}\left(s^{\prime}\right)+q} \\
& \leq \frac{\beta_{i}\left(s^{\prime}\right)+1}{\beta_{N}\left(s^{\prime}\right)+1}-\frac{\beta_{i}\left(s^{\prime}\right)}{\beta_{N}\left(s^{\prime}\right)} .
\end{aligned}
$$

By (5.10), there exist $t, t^{\prime} \in\{1, \ldots, m\}$ such that $[z(s-1), z(s)] \subseteq[x(t-1), x(t)]$ and $\left[z\left(s^{\prime}-1\right), z\left(s^{\prime}\right)\right] \subseteq\left[x\left(t^{\prime}-1\right), x\left(t^{\prime}\right)\right]$ and moreover, $\beta_{i}(s)=\alpha_{i}(t)$ and $\beta_{i}\left(s^{\prime}\right)=$ $\alpha_{i}\left(t^{\prime}\right)$. Then, from (5.12), it directly follows that

$\frac{\alpha_{i}(t)}{\alpha_{N}(t)}-\frac{\alpha_{i}(t)-1}{\alpha_{N}(t)-1}=\frac{\beta_{i}(s)}{\beta_{N}(s)}-\frac{\beta_{i}(s)-1}{\beta_{N}(s)-1}<\frac{\beta_{i}\left(s^{\prime}\right)+1}{\beta_{N}\left(s^{\prime}\right)+1}-\frac{\beta_{i}\left(s^{\prime}\right)}{\beta_{N}\left(s^{\prime}\right)}=\frac{\beta_{i}\left(t^{\prime}\right)+1}{\beta_{N}\left(t^{\prime}\right)+1}-\frac{\beta_{i}\left(t^{\prime}\right)}{\beta_{N}\left(t^{\prime}\right)}$

and thus, player $i$ can improve. 
Table 5.5: Part of the marginal gain table.

The element in row $r$ and column $c$ is the value $M(r, c)$ which represents player $i$ 's marginal gain incurred on an interval of length 1 from claiming it $r$ instead of $r-1$ times, given that it is claimed $c$ times by all other players. Similarly, $M(r, c)$ represents player $i$ 's marginal loss incurred on an interval of length 1 from claiming it $r-1$ instead of $r$ times, given that it is claimed $c$ times by all other players.

\begin{tabular}{c|cccccccccc} 
& 0 & 1 & 2 & 3 & 4 & 5 & 6 & 7 & 8 & 9 \\
\hline 1 & 1 & $\frac{1}{2}$ & $\frac{1}{3}$ & $\frac{1}{4}$ & $\frac{1}{5}$ & $\frac{1}{6}$ & $\frac{1}{7}$ & $\frac{1}{8}$ & $\frac{1}{9}$ & $\frac{1}{10}$ \\
2 & 0 & $\frac{1}{6}$ & $\frac{1}{6}$ & $\frac{3}{20}$ & $\frac{2}{15}$ & $\frac{5}{42}$ & $\frac{3}{28}$ & $\frac{7}{72}$ & $\frac{4}{45}$ & $\frac{9}{110}$ \\
3 & 0 & $\frac{1}{12}$ & $\frac{1}{10}$ & $\frac{1}{10}$ & $\frac{2}{21}$ & $\frac{5}{56}$ & $\frac{1}{12}$ & $\frac{7}{90}$ & $\frac{4}{55}$ & $\frac{3}{44}$ \\
4 & 0 & $\frac{1}{20}$ & $\frac{1}{15}$ & $\frac{1}{14}$ & $\frac{1}{14}$ & $\frac{5}{72}$ & $\frac{1}{15}$ & $\frac{7}{110}$ & $\frac{2}{33}$ & $\frac{3}{52}$ \\
5 & 0 & $\frac{1}{30}$ & $\frac{1}{21}$ & $\frac{3}{56}$ & $\frac{1}{18}$ & $\frac{1}{18}$ & $\frac{3}{55}$ & $\frac{7}{132}$ & $\frac{2}{39}$ & $\frac{9}{182}$ \\
6 & 0 & $\frac{1}{42}$ & $\frac{1}{28}$ & $\frac{1}{24}$ & $\frac{2}{45}$ & $\frac{1}{22}$ & $\frac{1}{22}$ & $\frac{7}{156}$ & $\frac{4}{91}$ & $\frac{3}{70}$ \\
7 & 0 & $\frac{1}{56}$ & $\frac{1}{36}$ & $\frac{1}{30}$ & $\frac{2}{55}$ & $\frac{5}{132}$ & $\frac{1}{26}$ & $\frac{1}{26}$ & $\frac{4}{105}$ & $\frac{3}{80}$ \\
8 & 0 & $\frac{1}{72}$ & $\frac{1}{45}$ & $\frac{3}{110}$ & $\frac{1}{33}$ & $\frac{5}{156}$ & $\frac{3}{91}$ & $\frac{1}{30}$ & $\frac{1}{30}$ & $\frac{9}{272}$ \\
9 & 0 & $\frac{1}{90}$ & $\frac{1}{55}$ & $\frac{1}{44}$ & $\frac{1}{39}$ & $\frac{5}{182}$ & $\frac{1}{35}$ & $\frac{7}{240}$ & $\frac{1}{34}$ & $\frac{1}{34}$ \\
10 & 0 & $\frac{1}{110}$ & $\frac{1}{66}$ & $\frac{1}{52}$ & $\frac{2}{91}$ & $\frac{1}{42}$ & $\frac{1}{40}$ & $\frac{7}{272}$ & $\frac{4}{153}$ & $\frac{1}{38}$
\end{tabular}




\section{Nederlandse Samenvatting (Summary in Dutch)}

Zoals de titel "Risico Aversie en Evenwichten, Inter-temporele Transfers, Strategische Claims" al aangeeft, komen een aantal onderwerpen aan de orde in dit proefschrift. Het gedeelte "Risico Aversie en Evenwichten" heeft betrekking op Hoofdstuk 2 en 3. De term risico aversie is gerelateerd aan het gedrag van een beslisser in een keuze situatie onder onzekerheid. Anders gezegd, iemand is risicomijdend wanneer hij, als hij de keuze heeft uit twee investeringen met eenzelfde verwacht rendement in de toekomst maar met verschillende risico's, kiest voor de investering met het laagste risico. "Inter-temporele Transfers" en "Strategische Claims" hebben betrekking op respectievelijk Hoofdstuk 4 en 5. Ze geven een korte omschrijving hoe we het strategisch model definiëren op de coöperatieve spelen die in deze hoofdstukken worden behandeld.

Het proefschrift behandelt vier verschillende economische modellen: bimatrix spelen, ruileconomieën onder onzekerheid, dynamische coöperatieve spelen met overdraagbaar nut en bankroet spelen. In deze modellen bestuderen we het gedrag van actoren (spelers) in een 'conflict' of verdeelsituatie (spel). In dit proefschrift concentreren we ons voornamelijk op de niet-coöperatieve speltheorie. Dit betekent dat iedere speler een strategie verzameling heeft en dat de oplossing afhangt van de gekozen strategieën van alle spelers. Spelers kunnen communiceren met elkaar, maar ze kunnen geen bindende afspraken maken. We veronderstellen dat spelers rationeel handelen, wat inhoudt dat iedere speler, gegeven de genomen strategieën van de andere spelers, een strategie kiest zodanig dat hij zijn verwacht nut maximaliseert. Een strategieprofiel bestaat uit een strategie uit de strategie verzameling van iedere speler. We zijn 
geïnteresseerd in strategieprofielen waar, gegeven de gekozen strategieën van andere spelers, geen enkele speler zijn nut kan verhogen door zijn strategie aan te passen. De strategieën in deze strategieprofielen zijn als het ware met elkaar in balans. In speltheorie wordt deze situatie een evenwicht genoemd. We bestuderen evenwichten in de vier economische modellen. De focus is bij ieder model net iets anders.

In Hoofdstuk 2 bekijken we Nash evenwichten van bimatrix spelen. We vergelijken Nash evenwichten met eenzelfde support die varieëren in de mate van risico aversie van speler 2 . We bekijken evenwichten in 2x2-bimatrix spelen en evenwichten met efficiënt support in coördinatie spelen. We laten voor deze evenwichten zien in welke gevallen het zich voordoen als meer risicomijdend, speler 2 baadt dan wel schaadt.

Het onderwerp dat centraal staat in Hoofdstuk 3 zijn ruileconomieën onder onzekerheid gelimiteerd tot twee spelers, één product en twee mogelijke toestanden van de wereld. De onzekerheid komt voort uit het gegeven dat op het tijdstip waarop spelers een beslissing moeten nemen, de toestand van de wereld nog niet bekend is. Een voorbeeld is het afsluiten van een ziektekostenverzekering door een verzekeringnemer bij een verzekeraar. Op het moment van aanschaf van de verzekering is voor beide partijen niet bekend of de verzekeringnemer ziek zal worden of niet. We bestuderen het effect waarbij een speler wordt vervangen door een speler met een hogere mate van risico aversie. We laten zien in welke gevallen de andere speler profiteert dan wel verliest als hij geconfronteerd wordt met een meer risicomijdende opponent in een evenwichtssituatie. Als een speler meer risicomijdend wordt, dan schuift de evenwichtsallocatie op naar de zogenoemde zekerheidslijn van die speler. De zekerheidslijn van een speler bevat allocaties die een speler in beide toestanden van de wereld eenzelfde hoeveelheid van het product geven. Of de verschuiving van de evenwichtsallocatie de andere speler baadt dan wel schaadt, hangt af van de uitgangssituatie van beide spelers.

In een coöperatief spel met overdraagbaar nut, kortweg TU-spel genoemd, wordt impliciet verondersteld dat de opbrengsten van een coalitie kunnen worden uitgedrukt in een getal dat op iedere mogelijke manier kan worden verdeeld onder de spelers als de coalitie wordt gevormd. Een TU-spel beschrijft een éénmalige gebeurtenis. Omdat veel gebeurtenissen een inter-temporeel karakter hebben en dus meer dan eens plaatsvinden over tijd, bekijken we in Hoofdstuk 4 dynamische TU-spelen. Deze spelen bestaan uit een eindig aan- 
tal spelers, een eindige opeenvolging van TU-spelen en een profiel van intertemporele nutsfuncties. Iedere periode wordt een (beperkt) additieve oplossing toegepast op een TU-spel. Dit resulteert voor iedere speler in een stroom van uitbetalingen, die hij kan evalueren met zijn inter-temporele nutsfunctie. Een beperking van het beschreven statische kader is dat het geen rekening houdt met dynamische aspecten die gepaard kunnen gaan met een inter-temporele gebeurtenis. In Hoofdstuk 4 implementeren we één van deze dynamische aspecten, namelijk de mogelijkheid tot lenen en sparen, door te veronderstellen dat spelers uitbetalingen kunnen transfereren tussen periodes. De strategische mogelijkheden van individuele transfers tussen periodes modelleren we in een niet-coöperatief spel. We laten zien in welke gevallen een Nash evenwicht bestaat in dit niet-coöperatieve spel. Ook laten we zien dat de uitbetaling van een Nash evenwicht in dominante strategieën Pareto optimaal is. De uitbetaling van een evenwicht is Pareto optimaal wanneer er geen ander evenwicht is met een uitbetaling zodanig dat iemand er op vooruit gaat, zonder dat dit ten koste van iemand anders gaat.

In Hoofdstuk 5 modelleren we een een bankroet spel, ook wel claims probleem genoemd, als een niet-coöperatief spel. In een claims probleem heeft iedere speler een claim op een boedel, maar is de boedel niet groot genoeg om aan alle claims te voldoen. Een strategie bestaat uit een keuze van de speler hoe zijn claim te plaatsen op de boedel. We laten hierbij toe dat spelers delen van de boedel meer dan eens kunnen claimen. Een gedeelte dat door meerdere spelers wordt geclaimd, wordt onder de spelers proportioneel naar gelang het aantal claims verdeeld. We karakteriseren de uitbetaling aan de spelers in Nash evenwichten in claims problemen met claims kleiner dan de boedel. In de twee speler situatie resulteert deze uitbetaling in de welbekende minimale overlap regel. We laten zien dat als claims oneindig groot worden, de uitbetaling in het evenwicht naar de proportionele regel convergeert. We bekijken variaties op dit model waarbij de claim op verschillende manieren kan worden verdeeld over de boedel. In ieder Nash evenwicht is de uitbetaling aan de spelers gelijk aan de proportionele regel. 



\section{Bibliography}

[1] J. Alcalde, M. del Carmen Marco, and J.A. Silva. The Minimal Overlap Rule Revisited. Social Choice and Welfare, 31:109-128, 2008.

[2] K.J. Arrow. Essays in the Theory of Risk Bearing. Chicago: Markham Publishing Co., 1971.

[3] M. Atlamaz. Contests with Fixed Resources in Spatial Models. Mimeo, 2004.

[4] M. Atlamaz, C. Berden, H. Peters, and D. Vermeulen. Non-cooperative Solutions for Claims Problems. Mimeo, 2008.

[5] R. Aumann and M. Maschler. Game Theoretic Analysis of a Bankruptcy Problem from the Talmud. Journal of Economic Theory, 36:195-213, 1985.

[6] C. Berden. The Role of Individual Intertemporal Transfers in Dynamic TUGames. Mimeo, 2007.

[7] C. Berden and H. Peters. On the Effect of Risk Aversion in Bimatrix Games. Theory and Decision, 60:359-370, 2006.

[8] C. Berden and H. Peters. On the Effect of Risk Aversion in Two-Person, Two-State Finance Economies. The B.E. Journal of Theoretical Economics, 7: Iss. 1 (Topics), Article 47, 2007.

[9] M. Berliant. An Equilibrium Existence Result for an Economy with Land. Journal of Mathematical Economics, 14:53-56, 1985. 
[10] M. Berliant and T. Ten Raa. A Foundation of Location Theory: Consumer Preferences and Demand. Journal of Economic Theory, 44:336-353, 1988.

[11] M. Berliant, W. Thomson, and K. Dunz. On the Fair Division of a Heterogeneous Commodity. Journal of Mathematical Economics, 21:201-206, 1992.

[12] Y. Chun. A Non-cooperative Justification for Egalitarion Surplus Sharing. Mathematical Social Sciences, 17:245-261, 1989.

[13] L. Corchón and C. Herrero. A Decent Proposal. Spanish Economic Review, 6:107-125, 2004.

[14] N. Dagan, R. Serrano, and O. Volij. A Non-cooperative View of Consistent Bankruptcy Rules. Games and Economic Behavior, 18:55-72, 1997.

[15] G. Debreu. A Social Equilibrium Existence Theorem. Proceedings of the National Academy of Sciences of the U.S.A., 38:386-393, 1952.

[16] J.A. Filar and L.A. Petrosjan. Dynamic Cooperative Games. International Game Theory Review, 2:47-65, 2000.

[17] I. García-Jurado, J. González-Díaz, and A. Villar. A Non-cooperative Approach to Bankruptcy Problems. Spanish Economic Review, 8:189-197, 2006.

[18] C. Gollier. The Economics of Risk and Time. The MIT press, 2001.

[19] C. Hara, J. Huang, and C. Kuzmics. Representative Consumer's Risk Aversion and Efficient Risk-sharing Rules. Journal of Economic Theory.

[20] H. Hotelling. Stability in Competition. The Economic Journal, 39:41-57, 1929.

[21] Y. Kannai. Concavifiability and Constructions of Concave Utility Functions. Journal of Mathematical Economics, 4:1-56, 1977.

[22] R.E. Kihlstrom, A.E. Roth, and D. Schmeidler. Risk Aversion and Solutions to Nash's Bargaining Problem. In O. Moeschlin and D. Pallaschke, editors, Game Theory and Mathematical Economics.

[23] V. Köbberling and H. Peters. The Effect of Decision Weights in Bargaining Problems. Journal of Economic Theory, 110:154-175, 2003. 
[24] L. Kranich, A. Perea, and H. Peters. Dynamic Cooperative Games. Mimeo, 2000.

[25] L. Kranich, A. Perea, and H. Peters. Core Concepts for Dynamic TU Games. International Game Theory Review, 7:43-61, 2005.

[26] J. Legut, J.A.M. Potters, and S.H. Tijs. Economies with Land - A Game Theoretical Approach. Games and Economic Behavior, 6:416-430, 1994.

[27] S.F. LeRoy and J. Werner. Principles of Financial Economics. Cambridge University Press, 2001.

[28] J.F. Nash. Non-cooperative games. Annals of Mathematics, 54:286-295, 1951.

[29] B. O'Neill. A Problem of Rights Arbitration from the Talmud. Mathematical Social Sciences, 2:345-371, 1982.

[30] B. Peleg and P. Sudhölter. Introduction to the Theory of Cooperative Games. Boston: Kluwer Academic Publishers, 2003.

[31] J.W. Pratt. Risk Aversion in the Small and in the Large. Econometrica, 32:122-136, 1964.

[32] D. Schmeidler. The Nucleolus of a Characteristic Function Game. SIAM Journal on Applied Mathematics, 17:1163-1170, 1969.

[33] L.S. Shapley. A Value for n-Person Games. In A.W. Tucker and H.W. Kuhn, editors, Contributions to the Theory of Games II, pages 307-317. New Jersey: Princeton University Press.

[34] A. Takayama. Mathematical Economics. Cambridge University Press, 2001.

[35] W. Thomson. Axiomatic and Game-theoretic Analysis of Bankruptcy and Taxation Problems: a Survey. Mathematical Social Sciences, 45:249-297, 2003.

[36] J. von Neumann and O. Morgenstern. Theory of Games and Economic Behavior. Princeton University Press, 1944. 



\section{Curriculum Vitae}

Caroline Berden was born on March 2nd, 1980 in Weert. She attended high school at Bisschoppelijk College Weert from 1992 till 1998. In September of 1998 she started her studies Econometrics at Maastricht University. In 2002 she studied half a year at Universidad de Barcelona under the Erasmus exchange program, which was followed by a six month internship at the Dutch Central Bank in Amsterdam. She received her Master's Degree in Econometrics/ Mathematical Economics in October 2003. In April 2004 she started her PhD research at the Faculty of Economics and Business Administration at Maastricht University. 
